
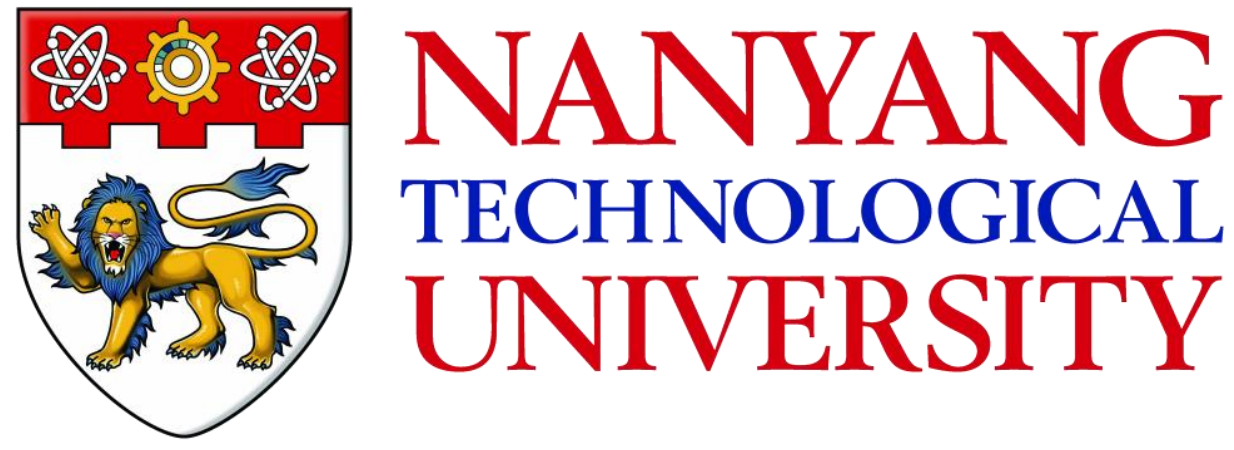

EXPLOITING RECEPTOR FLEXIBILITY TO IMPROVE THE ACCURACY OF STRUCTURE-BASED VIRTUAL SCREENING

YAW AWUNI

SCHOOL OF BIOLOGICAL SCIENCES

2016 


\title{
EXPLOITING RECEPTOR FLEXIBILITY TO IMPROVE THE ACCURACY OF STRUCTURE-BASED VIRTUAL SCREENING
}

\section{YAW AWUNI}

\section{SCHOOL OF BIOLOGICAL SCIENCES}

\author{
A thesis submitted to the Nanyang Technological University in \\ partial fulfillment of the requirement for the degree of \\ Doctor of Philosophy
}




\section{Acknowledgements}

First and foremost, I would like to express my heartfelt gratitude to my supervisor, A/Prof. $\mathrm{Mu}$ Yuguang, for giving me the opportunity to pursue my $\mathrm{Ph} . \mathrm{D}$ degree under his supervision. Without his patience, constant guidance, encouragement, and invaluable suggestions, this Ph.D thesis would not have been possible.

I am also grateful to Ass/Prof. Lu Lanyuan of the School of Biological Sciences (SBS), Nanyang Technological University (NTU) and Ass/Prof. Yang Liang of the Singapore Center on Environmental Life Sciences Engineering (SCELSE), NTU, for the constructive comments and insightful suggestions they made as my thesis advisory committee members.

I would also like to express my sincere gratitude to A/Prof. Robert C. Robinson and Dr. Shimin Jiang of the Institute of Molecular and Cell Biology (IMCB, A*STAR) for sacrificing their time and resources to provide the X-ray structures reported in Chapter 4 of this thesis. I also appreciate their relentless contributions to the study reported in Chapter 5 of this thesis.

Special thanks also go to the current and past laboratory mates of mine for sharing with me their experiences. I am grateful to Mr. Liangzhen Zheng, Mr. Liu Yang, Mr. Justin Ng Tze Yang, Mr. Amr Ali Mokhtar Alhossary, Ms. Nafisa Mohammed, and Dr. Ning Lulu. I am also indeed thankful to Dr. Luo Di, Dr. Han Nanyu, Dr. Zhang Tong, Dr. Wang Yaofeng and Dr. Li Weifeng. 
Lastly, my profound gratitude goes to the Singapore International Graduate Award (SINGA), NTU and SBS for the financial support. 


\section{Table of contents}

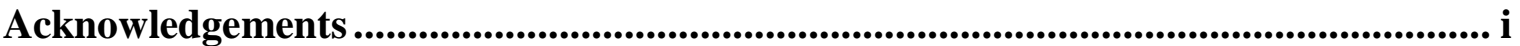

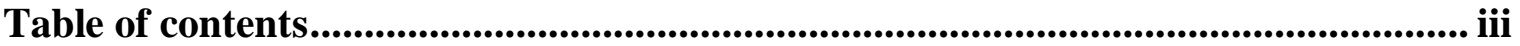

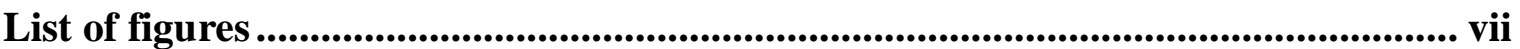

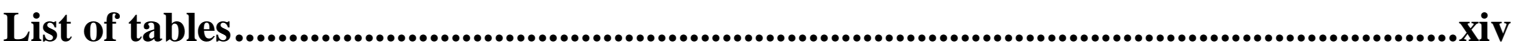

Abbreviations.........................................................................................................................XV

Abstract................................................................................................................................... xviii

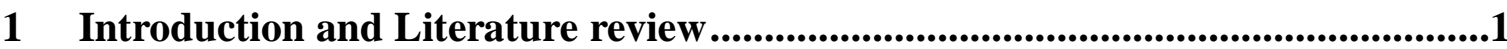

1.1 Historical perspective of drug discovery .............................................

1.2 Modern drug discovery process ............................................................2

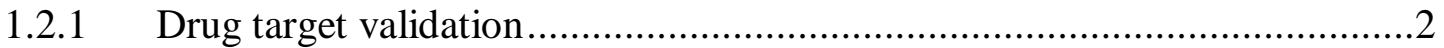

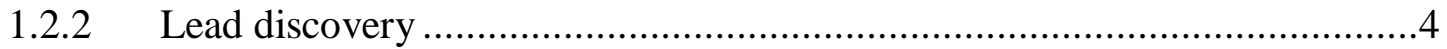

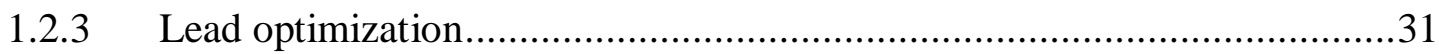

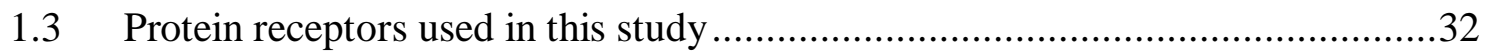

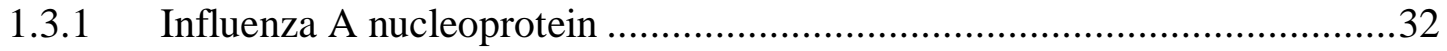

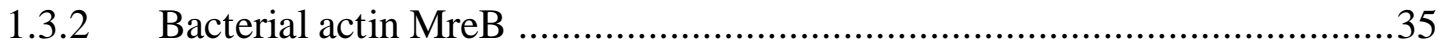

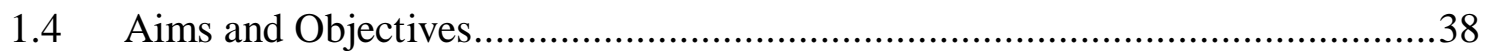

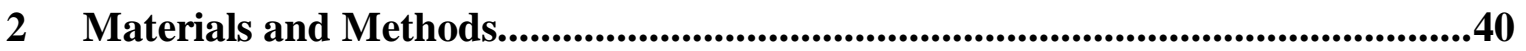

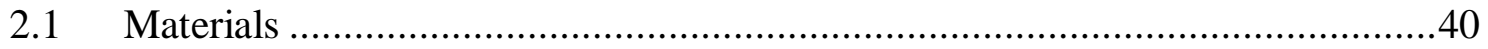




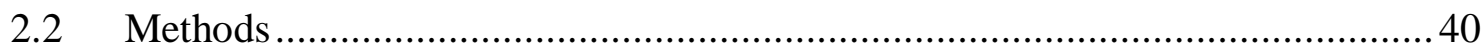

2.2.1 Preparation of influenza A nucleoprotein ..................................... 40

2.2.2 Preparation of MreB structures......................................................... 41

2.2.3 Molecular dynamics simulations ................................................ 42

2.2.4 Clustering for distinctive structures ........................................... 45

2.2.5 Docking and in silico screening ................................................ 47

2.2.6 Ligand selection criteria ...................................................... 49

3 Reduction of False Positives in Structure-Based Virtual Screening when Receptor Plasticity is Considered ...............................................................................551

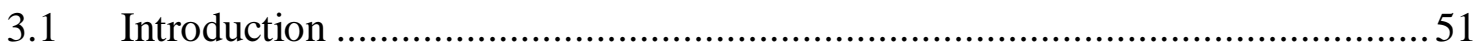

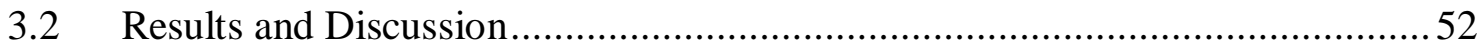

3.2.1 Selection by docking to multiple receptor conformations narrows down

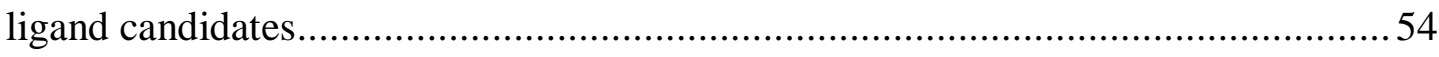

3.2.2 Molecule A compared to the high-affinity control .................................58

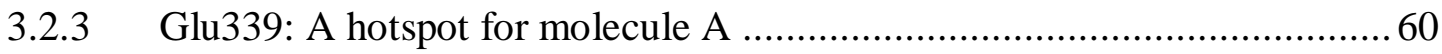

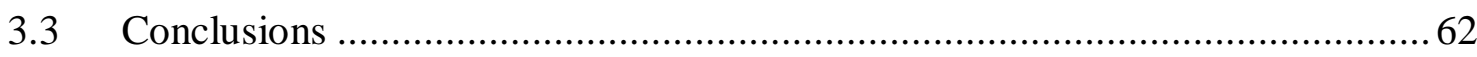

4 Validation of Computational Methods................................................................64

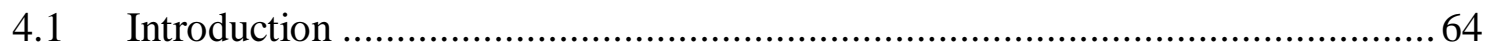

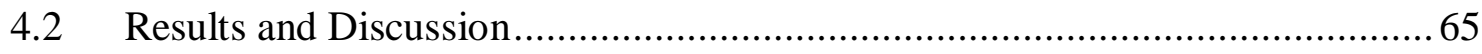


4.2.1 Selection of intersection molecules in top-ranked docking solutions identifies true binders .68

4.2.2 Analysis of binding modes of molecules A1, B2, and C3 ..........................71

4.2.3 Selection of intersection molecules in multiple receptor docking broadens the conformational and chemical space .73

4.3 Conclusions. .76

5 Exploring the A22-Bacterial Actin MreB Interaction through Molecular Dynamics Simulations ..................................................................................................................77

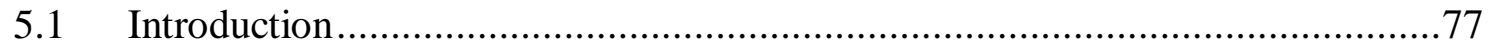

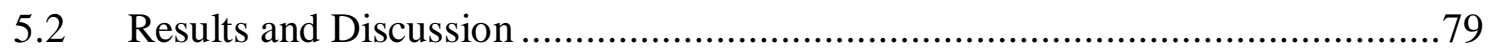

5.2.1 Validation of simulation protocol and systems ..........................................79

5.2.2 A22 interacts with the $\gamma$-phosphate group of ATP and AMPPNP ................81

5.2.3 Isothiourea group of A22 exhibits interaction preference in different

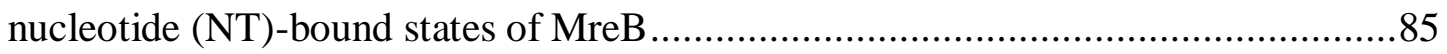

5.2.4 Water dynamics and ATP hydrolysis at the active site ...............................89

5.2.5 Stable hydrogen bonds are formed between $\mathrm{A} 22$ and $\mathrm{P}_{\mathrm{i}} \ldots \ldots \ldots \ldots \ldots \ldots \ldots \ldots \ldots \ldots . . . .91$

5.2.6 Proposed mechanism of inhibition of MreB by A22 ….............................94

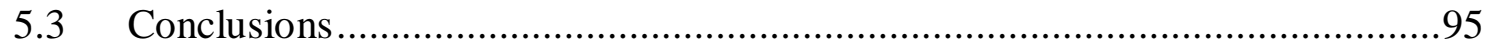

6 Discussion, Conclusions and Future work ................................................................97

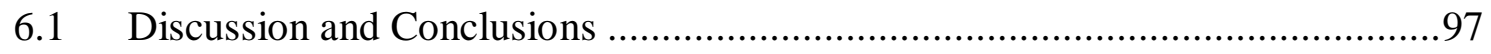




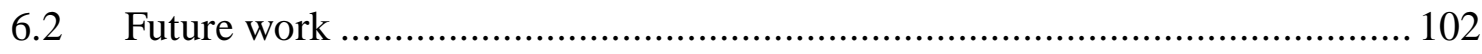

Appendix A................................................................................................................... 105

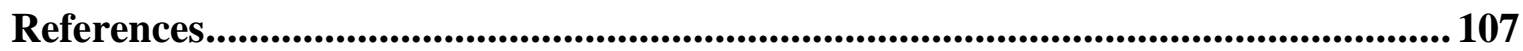




\section{List of figures}

Figure 1.1 Summary of the overall stages in structure-based drug discovery. Both computational and experimental stages are illustrated.

Figure 1.2 The binding energy landscape theory. Protein-ligand binding has a rugged funnel-like free energy landscape. .20

Figure 1.3 Illustration of soft docking approach. The black line indicates a hypothetical protein landscape. Green and red lines represent the protein-ligand interaction energy functions for two different ligands shown in corresponding colors, with arrows representing the computed binding energy in each approximation. Softening the L-J potential allow ligands of different shapes to be docked.(Modified and reprinted from reference [172]) with permission from John Wiley and Sons) .22

Figure 1.4 A graphical representation of multiple receptor docking strategies. (A) Illustration of averaged protein structure method. (B) Illustration of the superimposed structures method. (C) Illustration of separate multiple receptors method. .25

Figure 1.5 Molecular dynamics simulation is a bridge. (A) Between microscopic and macroscopic. (B) Between theory and experiment. (Figure is adapted from Ref. [187]) ..26 Figure 1.6 Cartoon representations of the structure of influenza A Nucleoprotein. (A) Monomeric structure of NP showing the head and body domains, T-loop, T-loop binding pocket and the RNA binding groove. (B) Trimeric structure of NP illustrating how NP-NP interaction occurs prior to RNA binding.

Figure 1.7 Inhibitors of influenza nucleoprotein. .34

Figure 1.8 Cartoon representations of MreB structures. (A) Monomeric structure of MreB. The nucleotide (NT) and A22 binding sites and the four subdomains of the protein are 
indicated. (B) The structure of a single filament of MreB. (C) The structure of a double

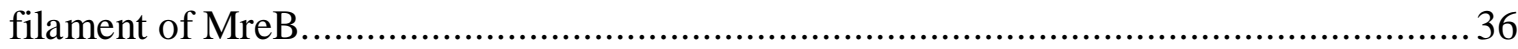

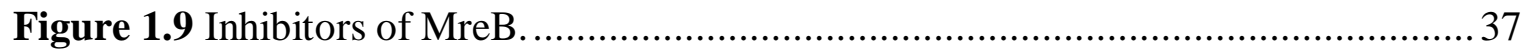

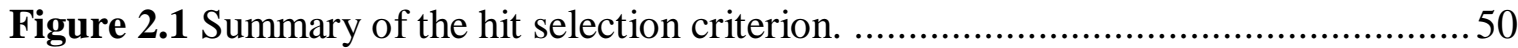

Figure 3.1 Crystal and MD receptor models of NP. (A) Receptor model for the T-loop binding pocket. The crystal structure is colored green. The MD structures $1,2,3,4$, and 5 are colored cyan, magenta, blue, yellow, and gray, respectively. (B) Receptor models for the RNA binding site. The crystal structure is colored green. The MD structures 1, 2, 3, and 4 are colored cyan, magenta, blue, and yellow, respectively.............................. 53

Figure 3.2 Molecules selected from the Otava PrimScreen1 library. (A) Selected molecules for the T-loop binding pocket. (B) Selected molecules for the RNA binding site. The LACS were not selected but are shown for illustrative purposes. ......................... 55

Figure 3.3 Number of intersection molecules in different number of conformations of the T-loop binding pocket and the RNA binding site of NP at different levels of comparison. (A) Number of intersection molecules for the T-loop binding pocket. (B) Number of intersection molecules for the RNA binding site. 57

Figure 3.4 Predicted binding modes of the high-affinity control (HAC) and molecule A in the T-loop pocket of the crystal structure of NP. Carbon atoms of the two molecules are colored in dark gray while carbon atoms of NP residues interacting with the molecules are colored in cyan. H-bonds are represented by black dashes. (A) The HAC forms H-bonds with Gly268, Glu339, and Thr390. Its aromatic ring is involved in aromatic- $\pi$ interactions with Phe304 and Trp330; (B) Molecule A forms H-bonds with Glu339, Ala387, Thr390, and Ser392. Its thiophene group forms ring stacking with Phe458. 59 
Figure 3.5 Molecular dynamics simulation data. RMSDs of the high-affinity control (HAC) and molecule A in complex with NP T-loop pocket are shown. The RMSDs were calculated on the molecules after least-squares fitting the protein.

Figure 3.6 Binding modes of molecule $\mathrm{A}$ in the distinctive T-loop binding pocket conformations of NP. The ability of the molecule to interact with Glu339 in all T-loop binding pocket conformations is illustrated. The binding mode of the molecule with the highest score in each conformation of the binding pocket was superimposed on that of the crystal structure. (A-E) Structure 1, 2, 3, 4, and 5 superimposed on the crystal structure. The crystal structure is colored in blue, MD generated structures are in yellow, carbon atoms of Glu339 and molecule $\mathbf{A}$ in the crystal structure are in green. Carbon atoms of Glu339 and molecule A in MD structures are colored in dark gray. H-bonds between Glu339 and molecule A are shown in black dashes.

Figure 4.1 Receptor models of MreB. The crystal is colored green, and the three MD structures are colored cyan, magenta, and blue.

Figure 4.2 Chemical structures of molecules selected for crystallographic studies. The control molecules are also shown.

Figure 4.3 Comparison of monomeric C. crescentus MreB (CcMreB) and T. maritima MreB (TmMreB). (A) Comparison of the primary sequence. Blue signifies exact match. (B) Comparison of the secondary and tertiary structural features. Green and magenta colors indicate C. crescentus MreB and T. maritima MreB respectively.

Figure 4.4 Electron density maps of molecules found in the active site of T. maritima MreB. (A-C) Electron density maps of molecules A1, B2, and C3, respectively. .70 
Figure 4.5 Crystal structures of MreB in complex with HAC4, molecules A1, B2, and C3. (A-D) Interactions between the active site residues of MreB and HAC4, molecule A1, molecule B2, and molecule C3. Balls in gray, blue, red, yellow, green, and cyan represent carbon, nitrogen, oxygen, sulfur, chlorine, and fluorine atoms, respectively. Bonds connecting the atoms of ligands are colored in magenta. Bonds connecting the atoms of amino acid residues are colored in orange. The dark red 'eyelashes' represent non-bonded contacts. H-bonds are shown in black dashes. .71

Figure 4.6 Orientations of HAC4, molecule A1, molecule B2, and molecule C3 in the presence of ADP. H-bonds are shown in dashes. .72

Figure 4.7 Comparison of MreB structures in molecule A1-bound, molecule B2-bound, molecule C3-bound, and HAC4-bound states. Molecule A1-bound, molecule B2-bound, molecule C3-bound, and HAC4-bound states are colored magenta, cyan, gray, and green, respectively. The insert shows the flexibility of the S6-H2 loop............................ 74 Figure 4.8 Surface representation of MreB in the HAC4-bound, molecule A1-bound, molecule B2-bound and molecule C3-bound states. The black circles indicate the shape of the active site. The HAC4-bound, molecule A1-bound, molecule B2-bound, and molecule C3-bound states are colored green, magenta, cyan, and gray, respectively. 75

Figure 5.1 Structures of A22, MP265, and MreB. (A) Structures of A22 and MP265 highlighting the (di) chlorobenzyl and isothiourea moieties. (B) Cartoon representation of the monomeric structure of $C$. crescentus MreB. The nucleotide (NT) and A22 binding sites and the four subdomains of the protein are indicated. 78

Figure 5.2 RMSDs of A22 in different nucleotide (NT)-bound states of MreB. (A-D) RMSDs of A22 in the ATP-A22-bound, AMPPNP-A22-bound, ADP-A22-bound and NT- 
free states of MreB, respectively. Black, red, and green colors represent first, second, and third simulations, respectively. The RMSDs were calculated on A22 after least-squares fitting the protein. .81

Figure 5.3 Interaction between A22, ATP, and MreB. The H-bonds formed between the isothiourea group of A22 and the $\gamma$-phosphate of ATP are indicated by black dashes. The arrow indicates that the isothiourea group can flip up or down around the sulfur atom. ...82

Figure 5.4 Minimum distance between heavy atoms of the isothiourea group of A22 and the carboxyl group of Glu140 in the ATP-A22-bound MreB. Black, red, and green traces represent the minimum distances from the first, second, and third simulations, respectively.

Figure 5.5 Crystal structures of $C$. crescentus MreB colored by b-factor. (A) The AMPPNP-MP265-bound state (PBD ID: 4CZK). (B) The ADP-A22-bound state (PBD ID: $4 \mathrm{CZG})$. The isothiourea groups are circled. .84

Figure 5.6 Data for H-bond formation probabilities between A22 and Glu140. (A-D) Probabilities of H-bond formation between A22 and Glu140 in the ATP-A22-bound, AMPPNP-A22-bound, ADP-A22-bound, and nucleotide (NT)-free states of MreB. Black, red, and green plots represent probabilities from the first, second, and third simulations, respectively. The criterion for selecting H-bonds was H-bond length not exceeding $3.5 \AA$. The probabilities were calculated after every $5 \mathrm{~ns}$

Figure 5.7 Data for H-bond formation probabilities between A22 and nucleotides. (A-C) Probabilities of H-bond formation between A22 and phosphate in the ATP-A22-bound, AMPPNP-A22-bound, and ADP-A22-bound states of MreB. Black, red, and green plots represent probabilities from the first, second, and third simulations, respectively. The 
criterion for selecting H-bonds was H-bond length not exceeding $3.5 \AA$ A The probabilities were calculated after every $5 \mathrm{~ns}$

Figure 5.8 Averaged probabilities of H-bond formation by A22. The black square symbols represent averaged probabilities of H-bond formation between A22 and Glu140, and the red circle symbols represent averaged probabilities of $\mathrm{H}$-bond formation between A22 and phosphate groups in different nucleotide (NT)-bound states of MreB. Each data point is an average of probabilities from three simulations. 88

Figure 5.9 Time spent by water molecules in the catalytic zone of MreB. (A) Time spent by water molecules in the catalytic zone of the ATP-A22-bound state. (B) Time spent by water molecules in the catalytic zone of the ATP-bound state. The catalytic zone is defined as the intersection of two spheres with the radii of $3.9 \AA$ and $3 \AA$ around the atoms $\mathrm{P} \gamma$ of ATP and OE1 of Glu140, respectively. The y-axis (H20 label) represents the numbering of the water molecules found in the catalytic zone. 90

Figure 5.10 Configuration of long-staying water molecules at the active site. " $d$ " is the distance between the atoms $\mathrm{O}_{\mathrm{w}}$ to $\mathrm{P}_{\gamma}$ " " $\alpha$ " is the angle defined by atoms $\mathrm{O}_{\mathrm{w}}, \mathrm{P}_{\gamma}$, and $\mathrm{O} 3_{\beta}$.

91

Figure 5.11 Interaction between $A 22$ and the excised $\gamma$-phosphate $\left(\mathrm{P}_{\mathrm{i}}\right)$ of ATP. The Hbonds are indicated by black dashes. 92

Figure 5.12 Simulation data for the ADP-Pi-bound state of MreB in the presence (A22+) and absence (A22-) of A22. (A-C) Distances between atoms $\mathrm{P}_{\gamma \mathrm{i}}$ of the $\mathrm{P}_{\mathrm{i}}$ and $\mathrm{C}_{\mathrm{i}}$ of $\mathrm{A} 22$, and between $\mathrm{P}_{\gamma \mathrm{i}}$ and $\mathrm{P}_{\beta}$ of ADP in three independent $\mathrm{A} 22+$ and $\mathrm{A} 22-$ simulations. The black, red, and green traces represent the distances between $\mathrm{P}_{\gamma \mathrm{i}}$ and $\mathrm{C}_{\mathrm{i}}$ in the $\mathrm{A} 22+$ 
simulations, $\mathrm{P}_{\gamma \mathrm{i}}$ and $\mathrm{P}_{\beta}$ in the $\mathrm{A} 22+$ simulations, and $\mathrm{P}_{\gamma \mathrm{i}}$ and $\mathrm{P}_{\beta}$ in the $\mathrm{A} 22$ - simulations, respectively.

Figure 5.13 Model for the effects of A22. (1) A22 forms H-bonds with the $\gamma$-phosphate of ATP. (2) The free catalytic Glu140 and water molecules trigger the hydrolysis of ATP to generate ADP and $\mathrm{P}_{\mathrm{i}}$. (3) The H-bonds between A22 and $\mathrm{P}_{\mathrm{i}}$ slow the release the release of the $\mathrm{P}_{\mathrm{i}}$. H-bonds are indicated by black dashes. .95 


\section{List of tables}

Table 1.1 List of common docking programs.(Modified and reprinted from reference[54] with permission from Bentham Science Publishers) ……............................................ 8

Table 2.1 Table summary of MD simulations of drug-free structures..............................43

Table 2.2 Table summary of MD simulations of drug-bound structures..........................45

Table 2.3 Control molecules and their experimentally determined affinities/activities....48

Table 3.1 Pattern of selection of molecules from the Otava Primscreen1 diversity library.

Table 3.2 Default GOLD scores of selected molecules in all receptor conformations.....58

Table 4.1 Pattern of selection of molecules from the Maybridge fragment library..........67

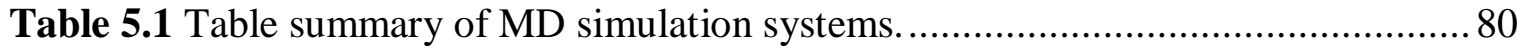

Table 5.2 Binding energy and quasi-harmonic entropy of A22 in different nucleotide

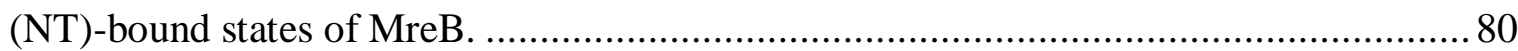




\section{Abbreviations}

$3 \mathrm{D}$

A22

ADP

Amber

AMPPNP

ATP

CADD Computer-aided drug discovery

CcMreB Caulobacter crescentus MreB

COX-2 Cyclooxygenase type 2

cryo-EM Cryo-electron microscopy

DAMPTC 2-[(3,4-dichlorophenyl)amino]-N-[3-(morpholin-4-yl)propyl]-1,3-thiazole-4-carboxamide

DS Discovery studio

FAON N-[2-[(3-Fluorophenyl) amino]-2-oxoethyl]nonanamide

GA Genetic algorithm

GAFF General amber force field

GMXPBSA Gromacs Poisson-Boltzmann surface area

GOLD Genetic optimization for ligand docking

Gromacs Groningen machine for chemical simulations

HAC High-affinity control

H-bond Hydrogen bond

HTS High throughput screening

K Kelvin 


\begin{tabular}{|c|c|}
\hline $\mathrm{kJ} / \mathrm{mol}$ & Kilojoules/mole \\
\hline LAC & Low-affinity control \\
\hline LBVS & Ligand-based virtual screening \\
\hline L-J & Leonard-Jones \\
\hline $\mathrm{MC}$ & Monte Carlo \\
\hline $\mathrm{MD}$ & Molecular dynamics \\
\hline MDCK & Madin-Darby canine kidney \\
\hline MF & Maybridge fragment \\
\hline MIC & Minimum inhibitory concentration \\
\hline MP265 & S-(4-chlorobenzyl) isothiourea \\
\hline MRC & Multiple receptor conformation \\
\hline mre & Murein cluster e \\
\hline NMR & Nuclear magnetic resonance \\
\hline NP & Nucleoprotein \\
\hline $\mathrm{ns}$ & Nanosecond \\
\hline NT & Nucleotide \\
\hline OP1D & Otava primeScreen1 diversity \\
\hline PA & Polymerase acidic protein \\
\hline PB1 & Polymerase basic protein 1 \\
\hline PB2 & Polymerase basic protein 2 \\
\hline PDB & Protein data bank \\
\hline PME & Particle Mesh Ewald \\
\hline ps & Picosecond \\
\hline
\end{tabular}




\begin{tabular}{ll} 
R.E.D & RESP ESP charge Derive \\
RCSB & Research collaboratory for structural bioinformatics \\
REMD & Replica exchange molecular dynamics \\
RMSD & Root mean square deviation \\
RNA & Ribonucleic acid \\
RNPs & Ribonucleoproteins \\
SAR & Structure-activity relationship \\
SBDD & Structure-based drug discovery \\
SBVS & Structure-based virtual screening \\
sdf & Standard data format \\
T-loop & Tail-loop \\
TmMreB & Thermotoga maritima MreB \\
US $\$$ & United States dollars \\
VS & Virtual screening \\
W & Mater \\
\hline M &
\end{tabular}




\section{Abstract}

Structure-based virtual screening is usually challenged by numerous false positives in the candidate drugs predicted by molecular docking tools. Here, on the basis of the binding energy landscape theory, a hypothesis that a true binder can bind to different conformations of the binding site favorably was put forth, and related strategies to defeat this challenge were devised; reducing false positives when receptor plasticity is considered. The receptors in the study are the influenza A nucleoprotein (NP) and the bacterial actin-like MreB. The structural flexibility of the receptors was explored by molecular dynamics (MD) simulations. The resultant distinctive structures and the respective crystal structures were used as receptor models in docking exercises in which two binding sites of NP, the tail-loop binding pocket and the RNA binding site, and the A22 binding site of MreB were targeted with molecule libraries using the GOLD software. In each case, the intersection ligands that were listed in the top-ranked molecules from all receptor models were selected. Such selection strategy successfully distinguished highaffinity and low-affinity control molecules added to the molecule libraries. This study provides an applicable approach for reducing false positives and selecting true ligands from molecule libraries.

Furthermore, X-ray crystallographic studies involving the molecules selected for the A22 binding site and MreB were carried to validate the selection criterion. Eleven hits, representing $0.037 \%$ of the total 30036 molecules making up the Maybridge fragment library were selected for crystallographic studies. Three of the hits were detected in the active site of MreB. The binding modes of these molecules are close to A22. The data 
obtained support the hypothesis that a true binder can bind to varying conformations of the binding site.

In addition, it was sought to understand the dynamics of the nucleotide-A22 binding site of the bacterial actin MreB. A22 is an antibiotic-like small molecule that perturbs the rod cell shape of bacteria and has been suggested to inhibit MreB by targeting ATP hydrolysis. However, without the elucidation of the structure of the ATP-A22-bound state of MreB, the mechanism of A22 inhibition is still not clear. Here conventional MD simulation was applied to explore the dynamics of the active site of MreB in complex with A22 and different nucleotides. The data obtained suggest that A22 may not function as an inhibitor of ATP hydrolysis. Analysis of the dynamics of water molecules in the MreB active site reveals that in the presence of A22 water molecules are able to occupy positions suitable for ATP hydrolysis. Thus these observations are consistent with a mechanism in which A22 affects MreB polymerization/depolymerization dynamics in part through slowing phosphate release rather than by inhibiting ATP hydrolysis. This is partially supported by the fact that the crystallographic structure of the ATP-bound state has not been solved in the presence of A22. These data can be incorporated in the design/development of the next generation of inhibitors of MreB. 



\section{Introduction and Literature review}

\subsection{Historical perspective of drug discovery}

Drug discovery is a step-by-step process through which a new substance with medicinal properties is discovered. It covers a broad scope of disciplines in biology, chemistry, and pharmacology. The historical foundation of drug discovery and development dates back to the early ages of human civilization. Plants, and to a lesser extend animal materials and minerals, were the main sources of early drugs [1], which were identified through serendipitous discovery, try-and-error experimentation or observation of the response by human and animal subjects after ingesting crude products. Examples of some of the sources of early drugs include Opium: induces sleep, relieves pain, cures diarrhea and improve sexual performance; Blue Lotus: induces lassitude and blissful sleep; Psilocybin: causes nausea and hallucinations, and Coca Leaf: has stimulating effects.

Owing to the advancement of analytical chemistry in the 1800 s, early scientific techniques found their way into the drug discovery process. This paved the way for the isolation of the active chemicals in the crude products, such as morphine [2] and papaverine [3] from opium extracts in 1817 and 1848 respectively. As the active but impure medicinal chemicals became accessible, emerging pharmaceutical companies confronted the challenge of producing the purified and standardized forms of those chemicals. Then on,

many drugs were discovered, tested, and synthesized in commercial quantities by pharmaceutical companies. The modern pharmaceutical industry came into existence after World War 1, and since then the application of scientific principles in drug discovery and development has been firmly established. 


\subsection{Modern drug discovery process}

Modern drug discovery involves a rather complicated and lengthy process that could be broadly categorized into target identification and validation, lead discovery, lead optimization, and clinical trial stages. On the average, the process takes 10-15 years to produce a pharmaceutical drug at an estimated cost of US\$ 800-1800 million [4-6].

\subsubsection{Drug target validation}

Once a drug target has been identified by any of the numerous methods available [7-10], one of the key steps to take at the beginning of any drug discovery project in order to avoid costly failure is target validation $[11,12]$. Target validation involves a careful examination of an identified potential drug target to confirm that it is indeed a feasible target. Some of the information about the target that target validation seeks to find include the role of the target in the life cycle of the pathogen; the uniqueness of the target to the pathogen; the presence of a possible drug binding site; the structural, biological, and chemical features of the possible drug binding site; and the expression, location, and accessibility of the target. Besides providing vital information about the druggability of the target, target validation could reveal very important features of the target that could inform decisions about the choice of methods and small-molecule databases to use in the drug discovery process. Target validation could be carried out by either experimental or computational means.

There are several experimental techniques that could be used to validate the druggability of a potential drug target. Some of the common experimental approaches used in target validation include (a) Sense reversal: this approach in rooted in the interruption of gene 
expression to reduce the quantity of the corresponding target protein, and identifying the physiological role of the target [11]. Examples of such approaches include gene knockouts [13], RNA interference [14-17], and antisense technology [18]. Carrying out target validation at the gene level, however, is disadvantaged by the fact that several genes produce different protein isoforms with different functions. Also, different proteins with different roles may arise from post-translational modifications of a given protein. Thus the interruption of gene expression could have undesired effects on the quantity and function of other proteins that are not the primary target, (b) Proteomics approach: this approach focuses on identifying the physiological role of the target by directly manipulating the target protein itself rather than the gene that expresses it $[11,19]$. The technique allows a specific form of a protein to be targeted, and avoids undesirable interferences in the expression of other proteins, and (c) Structure-based approach: by determining the primary structure (primary sequence) and/or the X-ray crystallographic, nuclear magnetic resonance (NMR) and cryo-electron microscopy (cryo-EM) three-dimensional (3D) structure of the target protein, useful information needed to validate its druggability could be extracted.

Target validation by computational means is the fastest and cheapest approach in the initial stages of drug discovery. With the structure (primary or 3D) of the target protein known, several computational models and approaches could be used to extract functional and druggable information [20-25]. If the primary sequence of the target is the only information available, comparative modeling (homology modeling and protein threading [26]) approaches could predict the function and the 3D structure by comparing the 
sequence with those of homologous structures whose functions and 3D structures are known. Otherwise, Ab intio- or de novo-protein modeling methods could be used to predict the $3 \mathrm{D}$ structure of the protein from scratch without comparing the sequence to previously solved structures. Once the X-ray, NMR, cyo-EM, homology or predicted 3D structure of the target protein is made available, there are several computer-based approaches that could identify possible drug binding pockets and determine surface descriptors that could be used to predict the druggability of the target [20-25].

\subsubsection{Lead discovery}

The lead discovery stage in drug discovery involves the identification of promising drugcandidate molecules mainly by screening large chemical databases against a whole pathogen or a specific drug target. The biochemical high throughput screening (HTS) and virtual screening (VS) are the two principal techniques applied in this phase. HTS is a rapid automated technology that can analyze databases of several organic molecules to find chemical actives. A typical HTS system usually consists of robotic handling systems, liquid handling systems, sensor systems and control software. Samples are loaded into an array of wells in microplates via the liquid handling systems. A robotic handling then moves the microplates through the system. The sensors in the sensor systems assess the samples in the microplate after a period of incubation. The control software organizes the whole process to ensure accuracy within a process and reproducibility between processes. One desirable attribute of HTS is that it does not require a prior knowledge of the drug binding site on the target protein. 
The term VS describes a computer-based process of analyzing a large database of small molecules with a goal of reducing the chemical space to a reasonable number of lead-like molecules that stand a good chance of becoming drug candidates of a biological structure. These lead-like molecules are then synthesized or purchased and tested. VS is divided into two main techniques, namely ligand-based (LBVS) and structure-based (SBVS) [27]. LBVS uses structure-activity information from known active compounds of a drug target to identify a new class of candidate drugs for experimental validation [28]. The main idea underlying the LBVS methods is that related structures have similar biological activity. Once known active compounds of the drug target are available, computed similarity measures, pharmacophores or descriptors from these actives can be used to retrieve other potentially active molecular entities from molecule databases. This method is particularly useful where the structure of the biological target is not available. LBVS is disadvantaged by the fact that there exist a weak relationship between structural similarity and activity [29]. Also, the relationship between structural similarity and activity depends on the similarity measure which could differ from one method to the other. Different similarity measures have been suggested $[30,31]$. Similarity search, substructure/superstructure search, quantitative structure-activity relationship (QSAR) models, pharmacophore modeling, and 3D shape matching are some of the techniques used in LBVS [32].

In SBVS, the database of molecules is screened against the biological target whereby the molecules are ranked on the basis of their estimated binding affinities or complementarity to the binding site. Knowledge of existing or known actives of the drug target is not required but its 3D structure is needed. The scoring of ligand poses is the most critical 
stage in SBVS, and determines its success [33, 34]. Accurate scoring of predicted ligandbinding poses in SBVS is quite challenging and thus makes it difficult to identify the most promising drug candidates. To date, it is challenging to parameterize some terms in scoring functions for accurate ranking of ligands. Molecular docking is the main technique used in SBVS.

A promising VS approach in recent years is an attempt to merge the most successful LBVS methods with SBVS [35]. The ultimate goal of this strategy is to combine chemical and structural information to enhance the chances of identifying the most promising drug candidates. Several studies have attempted to use this approach [33, 36-40], which requires the 3D structure and known active compounds of the receptor. The combination of LBVS and SBVS is usually done in a serial or parallel fashion. In the serial technique, the fastest and less refined steps are placed first followed by the slow and computational expensive ones. In the parallel combination approach, a comparison is made between the candidate drugs selected by both methods. Such candidate drugs are either compounds selected by each approach (complementary selection) or compounds selected by both methods (consensual selection). Unfortunately, due in part to the inability of common LBVS and SBVS combination methods to deliver superior performance attempts to merge the two have produced mixed results [41].

\subsubsection{Protein-ligand docking}

In view of the advancement of computer power and algorithms, SBVS has become a cheaper alternative to HTS in pharmaceutical research. Lately, SBVS has become popular because the 3D structures of proteins that play vital roles in the survival and 
multiplication of pathogens are rapidly becoming available in the protein data bank (PDB). There have been a growing number of successes involving SBVS [42-45].

SBVS employs the molecular docking technique, which predicts the possible ligand poses in the binding site of a receptor and ranks those poses on the basis of their estimated binding affinities. The majority of docking algorithms carry out two cardinal operations, namely "docking" and "scoring". They predict the structures of a protein-ligand complex and estimate the binding affinities. A number of protein-ligand conformations called poses or orientations are generated by a search/sampling algorithm in the "docking" phase of the operations. A scoring function then ranks the poses in the "scoring" phase. Ideally, the search algorithm, two most important features of which are the speed and efficiency in exploring the relevant structures of the protein-ligand complex [46], should be capable of adequately sampling the degrees of freedom of the protein-ligand complex to include the true binding conformation. The scoring function is also expected to rank the true binding conformation highest among all the sampled conformations. Currently, there are several docking programs available [47-54]. Table 1.1 presents a list of some of the most common protein-ligand docking tools released in the period 2006-2011 [54]. The programs are listed in order of the year of publication, with indications of the countries of origin and the original publications. 
Table 1.1 List of common docking programs.(Modified and reprinted from reference[54] with permission from Bentham Science Publishers)

\begin{tabular}{|c|c|c|c|}
\hline Program & Year & Country & References \\
\hline DOCK & 1988 & USA & {$[49,55,56]$} \\
\hline AutoDock & 1990 & USA & [57-59] \\
\hline SOFTDocking & 1991 & USA & {$[60]$} \\
\hline DockVision & 1992 & Canada & [61] \\
\hline LUDI & 1992 & Germany & [62] \\
\hline ADAM & 1994 & Japan & [63] \\
\hline FLOG & 1994 & USA & [64] \\
\hline SYSDOC & 1994 & USA & [65] \\
\hline DIVALI & 1995 & USA & [66] \\
\hline GOLD & 1995 & UK & {$[48,53]$} \\
\hline FlexX & 1996 & Germany & {$[50,67]$} \\
\hline Hammerhead & 1996 & USA & {$[68]$} \\
\hline LIGIN & 1996 & Israel| Germany & [69] \\
\hline FTDOCK & 1997 & UK & [70] \\
\hline ICM-Dock & 1997 & USA & [71] \\
\hline QXP & 1997 & USA & [72] \\
\hline PRO_LEADS & 1998 & UK & [73] \\
\hline SANDDOCK & 1998 & UK & [74] \\
\hline MCDOCK & 1999 & USA & [75] \\
\hline PRODOCK & 1999 & USA & [76] \\
\hline DARWIN & 2000 & USA & [77] \\
\hline EUDOC & 2001 & USA & [78] \\
\hline FlexE & 2001 & Germany & [79] \\
\hline FDS & 2003 & UK & {$[80]$} \\
\hline FRED & 2003 & USAUK & [81] \\
\hline LigandFit & 2003 & USA & [82] \\
\hline PhDOCK & 2003 & USA & [83] \\
\hline Surflex & 2003 & USA & [84] \\
\hline GEMDOCK & 2004 & Taiwan & [85] \\
\hline Glide & 2004 & USA & {$[51,52]$} \\
\hline ProPose & 2004 & Germany & [86] \\
\hline YUCCA & 2005 & USA & [87] \\
\hline MolDock & 2006 & Denmark & [88] \\
\hline PLANTS & 2006 & Belgium / Germany & [89] \\
\hline PSI-DOCK & 2006 & China & [90] \\
\hline eHiTS & 2006 & Canada $\mid$ UK & [91] \\
\hline EADock & 2007 & Switzerland & [92] \\
\hline FLIPDock & 2007 & USA & [93] \\
\hline MDock & 2007 & USA & [94] \\
\hline PSO@AUTODOCK & 2007 & Germany & [95] \\
\hline ParDOCK & 2007 & India & [96] \\
\hline SODOCK & 2007 & Taiwan & [97] \\
\hline Lead finder & 2008 & Russia | Canada & [98] \\
\hline MS-DOCK & 2008 & France & [99] \\
\hline Q-Dock & 2008 & USA & [100] \\
\hline MADAMM & 2009 & Portugal & [101] \\
\hline AutoDockVina & 2010 & USA & [102] \\
\hline AADS & 2011 & India & [103] \\
\hline BetaDock & 2011 & South Korea & [104] \\
\hline LigDockCSA & 2011 & South Korea & [105] \\
\hline PythDock & 2011 & South Korea & [106] \\
\hline VoteDock & 2011 & Poland & [107] \\
\hline
\end{tabular}


Regardless of the challenges of protein-ligand docking, including poor treatment of solvation/desolvation [108, 109], entropic effects [108, 109], and full plasticity of the protein receptor (receptor reorganization) [110], it has virtually become an integral part of the drug discovery process partly because it provides rapid and cost-effective computational means for screening large molecule databases $[111,112]$. With the 3D structure of a protein receptor known, molecular docking can be used to screen large ligand libraries for potential inhibitors or lead molecules. Nonetheless, the process of finding a lead molecule by molecular docking and eventually developing it into a pharmaceutical drug is a prolonged and capital intensive process [4-6]. Figure 1.1 illustrates the lengthy combination of SBVS and chemical biology methods in a modern drug discovery process. A good docking, scoring, and hits selection protocol may facilitate the identification of a lead molecule or a true binder but it may take several years (10-15 averagely) to optimize its pharmacological properties to meet the requirements of a pharmaceutical drug.

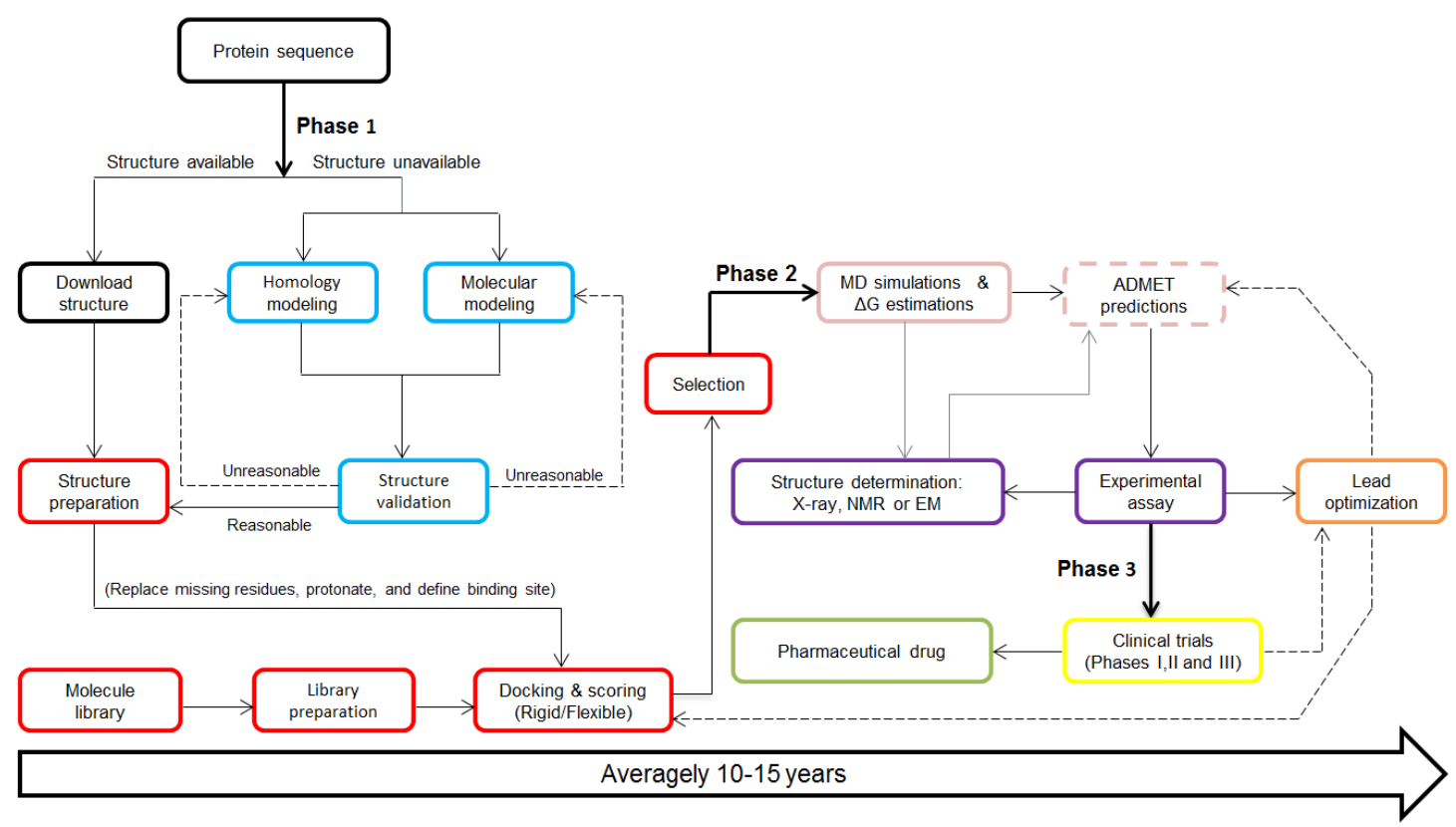

Figure 1.1 Summary of the overall stages in structure-based drug discovery. Both computational and experimental stages are illustrated. 


\section{Search/sampling algorithms}

A number of search algorithms have been developed and incorporated into molecular docking. Some of the most common sampling algorithms include matching algorithms [113-115], multiple copy simultaneous search algorithms [116, 117], LUDI [118], incremental construction algorithms [50, 119, 120], Monte Carlo (MC) algorithms [61, 121], genetic algorithms (GAs) [48, 58, 122], and molecular dynamics (MD) algorithms. Matching algorithms [113-115] are geometry-based methods that represent both the protein receptor and the ligand as pharmacophores and rely on shape and chemical characteristics to map the ligand into the binding site. This is then followed by the matching of calculated pharmacophore distances within the receptor and ligand whereby the distance matrix between the pharmacophore and the corresponding ligand atoms determine new ligand conformations. During the match, hydrogen bond (H-bond) donors and acceptors, as well as other chemical properties, can be taken into account. Matching algorithms are suitable for screening large databases because of their speed. Dock [123], FLOG [64], LibDOCK [124], and SANDOCK [74] are examples of docking programs the use matching algorithms.

Multiple copy simultaneous search $[116,117]$ is a fragment-based approach for the de novo design of new ligands and optimization of the binding affinities of existing ligands. In this approach, about 1000-5000 duplicates of a functional group of the ligand are generated and randomly placed in the binding site of the protein and put through simultaneous energy optimization and/or quenched MD. Only the interaction between copies of the functional group and the protein are accepted. Interactions among copies are 
rejected. Based on the interaction energies, a set of energetically favorable binding sites and functional group orientations are identified. By repeating this process with different functional groups, the binding site of the receptor is mapped. New compounds that perfectly fit into the binding site are designed by linking the corresponding functional groups.

LUDI [118] is also a fragment-based strategy for de novo design of ligands and focuses on potential H-bonds and hydrophobic interactions between the ligand and protein. LUDI relies mainly on interaction sites, which are distinct positions in space appropriate for $\mathrm{H}$ bonding and hydrophobic interactions [118]. The fragment is fitted on a set of interaction sites that are generated by searching the database or using rules and evaluated by distance criteria. After the evaluation step, some selected or all the fitted fragments could be connected to generate a parent molecule.

Incremental construction algorithms $[50,119,120]$ involve systematic search-based methods that build the ligand in the binding site. First and foremost, the rotatable bonds of the ligand are broken to generate several fragments. Then one of these fragments, usually the largest or the one with important functional role or interaction with the protein, is docked into the binding site to serve as an anchor. Once the anchor is properly positioned in the binding site, the other fragments are carefully added incrementally to generate a ligand that properly fits into the binding cavity. The phase space of the ligand is explored by generating different orientations of the fragments to find the best fit. This method is used by DOCK 4.0 [49], FleXX [50], Hammerhead [68], SLIDE [125], and eHiTS [126] docking programs. 
The MC [61, 121] methods involve a stochastic/random search whereby the conformational space is explored by randomly modifying the ligand or a group of ligands. The poses of a ligand are generated by rotation of bonds and translation or rotation of rigid bodies. An energy-based criterion is applied to determine which conformation is accepted or rejected. Any conformation of the ligand that is accepted is saved and subjected to further modification and energy evaluation to generate another one. The process continues until a pre-determined conformational sample size is attained. One main advantage of the MC method over MD-based methods is that the conformational change of the ligand is so large to allow it to cross the energy barriers on the potential energy surface. Examples of programs applying MC search are QXP [72], ICM [127], Affinity [128], and an earlier version of AutoDock [57].

The GAs $[48,58,122]$ represent another popular class of stochastic search methods. The fundamental concept of GA methods originates from Darwin's theory of evolution. The technique involves encoding the degrees of freedom of a ligand by binary strings referred to as 'genes'. The pose of the ligand is represented by a collection of its genes called 'chromosome'. Two main operations, namely mutations and crossover take place in GA methods. During the mutation phase, random changes are made to the genes leading to the generation of other chromosomes. In the crossover phase, there is an exchange of genes between two chromosomes. Thus a new ligand conformation is generated when the genetic operators affect the genes. The new ligands that attain binding energies above a threshold, upon evaluation by a scoring function, are the 'survivors' to be used for the 
next generation. AutoDock [58], GOLD [48, 53, 129], DIVALI [130], and DARWIN [77] are docking programs that apply GA.

In MD algorithms, the position of each atom in the simulation system is independently adjusted in the field of all other atoms. This technique allows the conformational degrees of freedom of both the protein and ligand to be explored. The main challenge associated with MD-based methods is the possibility of poor conformational sampling within a reasonable time scale as a result of ligands getting trapped in local minima.

\section{Scoring functions}

Several scoring functions are available for scoring docking poses. These are usually categorized into force-field-based, empirical-based, and knowledge-based [33]. Apart from accuracy, a scoring function should be fast enough to allow for the scoring of a large number of possible docking solutions. However, making modifications to scoring functions to achieve speed is dicey, as accuracy could be compromised. Common examples of scoring functions include GoldScore [48, 53, 129], ChemScore [131], DScore [67], DrugScore [132, 133], Fresno[134], F-Score [50], G-Score[67], XSCORE[135], and SMoG score [136].

Force-field-based scoring functions [137-139] basically compute the sum of the nonbonded interactions (electrostatics and van der Waals interactions) between the protein and ligand to evaluate the binding energy. The electrostatic terms are computed by applying Coulomb's law and formulation whereby the interacting particles are represented as point charges. Since point charge calculations are challenged by their inability to model the real biological environment of the protein, the contribution of charge-charge 
interactions to the binding energy is regulated by a distance-dependent dielectric function. The van der Waal's interactions are represented by the Lennard-Jones (L-J) potential function. The 'hardness' of the potential defines how close the contact between the protein and ligand atoms could be. Adopting varying parameter sets for the L-J potential can regulate the 'hardness' of the potential to determine the allowable contact. Higher terms such as 12-6 L-J potential make the potential harder by increasing repulsion and will penalize close contacts between the atoms of the protein and ligand. Terms such as 84 L-J potential make the potential softer to allow close contacts between the protein and ligand. Force-field-based scoring functions are challenged by low computational speed because the non-bonded terms are affected by long-range interactions. A distance cut-off scheme is used to overcome this challenge, but this also underestimates long-range effects in the protein-ligand interaction. There are extensions of force-field based scoring functions in docking programs such as Dock [49, 120, 123, 140], GOLD, and AutoDock [58] to consider contributions of H-bonds, solvation, and entropy to the binding energy calculations. However, these programs differ in the treatment of $\mathrm{H}$-bonds and the form of the energy function used.

The empirical scoring functions [141-145] rely on the notion that binding energy can be estimated by summing up separate uncorrelated terms. The binding energy is decomposed into a number of energy terms, including H-bond, ionic interaction, hydrophobic effect, and binding entropy. The overall score is obtained as the sum of the product of each energy component and a corresponding coefficient. The coefficients are obtained from a regression analysis on a collection of receptor-ligand complexes with known binding 
affinities. A major challenge with the empirical-based scoring functions emanates from the fact that the terms included and treatment of terms varies from one docking program to the other. Also, although these scoring functions have relatively simple energy terms, it is not clear as to how accurate they are beyond the training set. LUDI [118], PLP [142, 143, 146] and ChemScore [131] originate from empirical scoring functions.

The knowledge-based scoring functions [147-152] are based on the assumption that a more favorable interaction has a high frequency of occurrence. Statistical analysis of crystal structures of protein-ligand complexes is carried to acquire the interatomic contact frequencies and/or distances between the protein and ligand. The frequency distributions are converted into pairwise atom-type potentials. To calculate the final score, a cut-off is chosen within which preferred contacts are favored and repulsive interactions penalized. Knowledge-based scoring functions are simple and suitable for screening large molecule databases. They also provide a means to model rare interactions such as sulfur-aromatic or cation-pi, which pose some challenges to empirical methods. Due to the bias in the selection of training sets, the parameters obtained may not be suitable for all interactions. Interactions involving metals and halogens may be poorly handled by these scoring functions. Examples of empirical-based scoring functions include PMF [147], DrugScore [133], Smog [136], and Bleep [148]. They differ based on the size of training sets, the form of the function, atom type definition, cut-off distance or other parameters.

\section{The AutoDock program}

AutoDock [58] uses the Lamarckian GA and empirical free energy scoring function to predict and evaluate the poses of a ligand in the binding site. The Lamarckian GA 
algorithm is a combination of the GA method and an adaptive local search method [58]. The AutoDock scoring function, which is a semiempirical free energy force field, is an extension of force-field based scoring functions to consider van der Waals interactions, electrostatic interactions, H-bonds, and entropy in the binding energy estimation. The evaluation of the energy of protein-ligand binding is divided into two phases. The first step involves estimation of the intramolecular energies associated with the transitions from the unbound states to the protein-ligand complex. This is then followed by the evaluation of the intermolecular energies associated with the interaction between the protein and the ligand to form the complex. Six pair-wise evaluations (V), as well as the conformational entropy that is lost upon ligand binding ( $\Delta$ Sconf), are considered by the force field. Thus for a protein $P$ and a ligand $L$, the binding energy $\Delta \mathrm{G}$ is of the form:

$\Delta G=\left(V_{\text {bound }}^{L-L}-V_{\text {unbound }}^{L-L}\right)+\left(V_{\text {bound }}^{P-P}-V_{\text {unbound }}^{P-P}\right)+\left(V_{\text {bound }}^{P-L}-V_{\text {unbound }}^{P-L}+\Delta S_{\text {conf }}\right)$

For two atoms $i$ and $j$, if $W$ s are weighting constants to calibrate the empirical free energy, the pair-wise atomic energy $(V)$ is obtained as the sum of van der Waals (repulsion/dispersion), hydrogen bonding, electrostatics, and desolvation:

$V=W_{v d w} \sum_{i, j}\left(\frac{A_{i j}}{r_{i j}^{12}}-\frac{B_{i j}}{r_{i j}^{6}}\right)+W_{\text {hbond }} \sum_{i j} E(t)\left(\frac{C_{i j}}{r_{i j}^{12}}-\frac{D_{i j}}{r_{i j}^{10}}\right)+W_{e l e c} \sum_{i j} \frac{q_{i} q_{j}}{e\left(r_{i j}\right) r_{i j}}+W_{s o l} \sum_{i j}\left(S_{i} V_{j}+S_{j} V_{i}\right) e^{-\frac{r_{i j}^{2}}{2 \sigma^{2}}}$

\section{The GOLD docking program}

The GOLD docking program $[48,53,129]$ is one of the most common docking tools available [54]. GOLD employs a GA to account for full flexibility of the ligand and 
partial protein conformational plasticity. A study involving a dataset of 100 protein-ligand complexes sampled from the PDB showed that GOLD is good at reproducing experimental ligand binding poses [48]. The program meets the fundamental idea that solvent molecules that are weakly bound must be displaced by the ligand to permit the formation of bonds between the ligand and the protein. The GOLD program is made up of three main components, namely a scoring function, a technique for putting the ligand in the binding site, and a sampling algorithm [48, 53, 129].

\section{The GOLD scoring function}

GOLD uses a molecular mechanics-like function referred to as GoldScore to rank binding poses. The GoldScore is made up of four terms as shown in equation 3 [129].

GoldScore $=S_{h b_{-} e x t}+S_{v d w_{-} e x t}+S_{h b_{-} i n t}+S_{v d w_{-} i n t}$

$S_{h b_{-} \text {ext }}$ is the score for H-bonds between the protein and ligand.

$S_{v d w_{-} e x t}$ is the score for the van der Waals interactions between the protein and ligand.

$S_{h b_{-} i n t}$ is the score for the intramolecular H-bonds in the ligand. This term is turned off by default, as this is found to give the best results [129].

$S_{v d w_{-} i n t}$ is the contribution to the GoldScore as a result of intramolecular strain within the ligand. 


\section{Generating a ligand pose by GOLD}

A method that relies on fitting points is used by GOLD to position the ligand in the binding site. First, the program places fitting points on H-bonding groups on the protein and the ligand. This is then followed by the mapping of H-bond acceptor points on the ligand on $\mathrm{H}$-bond donor points in the protein and vice versa. Also, hydrophobic fitting points are generated in the binding site followed by the mapping of the $\mathrm{CH}$ groups of the ligand on those points and vice versa [129].

\section{The GOLD sampling algorithm}

GOLD uses a GA to explore the possible conformations of the protein-ligand complex. In the GA, the dihedral angles of the rotatable bonds of the ligand, ring geometries of the ligand, dihedral angles of $\mathrm{OH}$ and $\mathrm{NH}_{3}{ }^{+}$groups of the protein, and the mappings of the fitting points are optimized [48, 53, 129].

Randomly, a population of predicted docking solutions referred to as 'chromosomes' is put together. A 'chromosome' carries the relevant information required for (a) mapping the H-bond atoms of the ligand onto those of the protein and vice versa, (b) mapping of the hydrophobic points on the ligand onto the proteins and vice versa, and (c) the conformation around flexible bonds of the ligand and the $\mathrm{OH}$ groups of the protein. Based on the binding affinity, the 'chromosomes' in the population are scored and ranked accordingly. This is then followed by an iterative optimization of the population of 'chromosomes'. At each step of the optimization process, a daughter 'chromosome' may be formed as a result of a point mutation in a parent or the mating of two parents. There is 
a bias skewed towards healthier members, 'chromosomes' with good fitness scores, of a population for the selection of parent chromosomes for mutation and mating [48, 53, 129].

\subsection{Treatment of flexibility in protein-ligand docking}

Both the protein receptor and ligand have conformational degrees of freedom in the 3D space. Therefore, accounting for flexibility in protein-ligand docking is a fundamental challenge to search algorithms. The computational cost involved in exploring the phase spaces of the protein and ligand depends on the degrees of freedom included in the search. Broadly, rigid-body, flexible-ligand, and flexible ligand-flexible receptor docking are strategies that have been devised to handle this limitation. In the rigid-body algorithms, the receptor and the ligand are both treated as rigid while only the six degrees of freedom of translation and rotation are explored. The flexible-ligand algorithms treat the receptor as rigid while the flexibility of the ligand is explored. The flexible ligand-flexible receptor methods try to account for the flexibility of both the ligand and the receptor. Most docking programs are able to account for the conformational degrees of freedom of the ligand. However, treating the full plasticity of the protein receptor is a herculean task for these programs. Some modern docking tools attempt to incorporate partial flexibility of the receptor by accounting for slight movements of protein side chains and backbones [153-158].

Protein-ligand binding has a rugged funnel-like energy landscape (Figure 1.2) that allows multiple interaction patterns [159]. This is rooted in the fact that proteins, like ligands, can assume different binding modes as a result of structural plasticity $[160,161]$. The focus here is not on the induced-fit mechanism whereby ligand binding causes a change in the 
conformation of the receptor. Instead, the emphasis is placed on the internal freedom of motions that occur in the holo and apo form of the protein receptor.

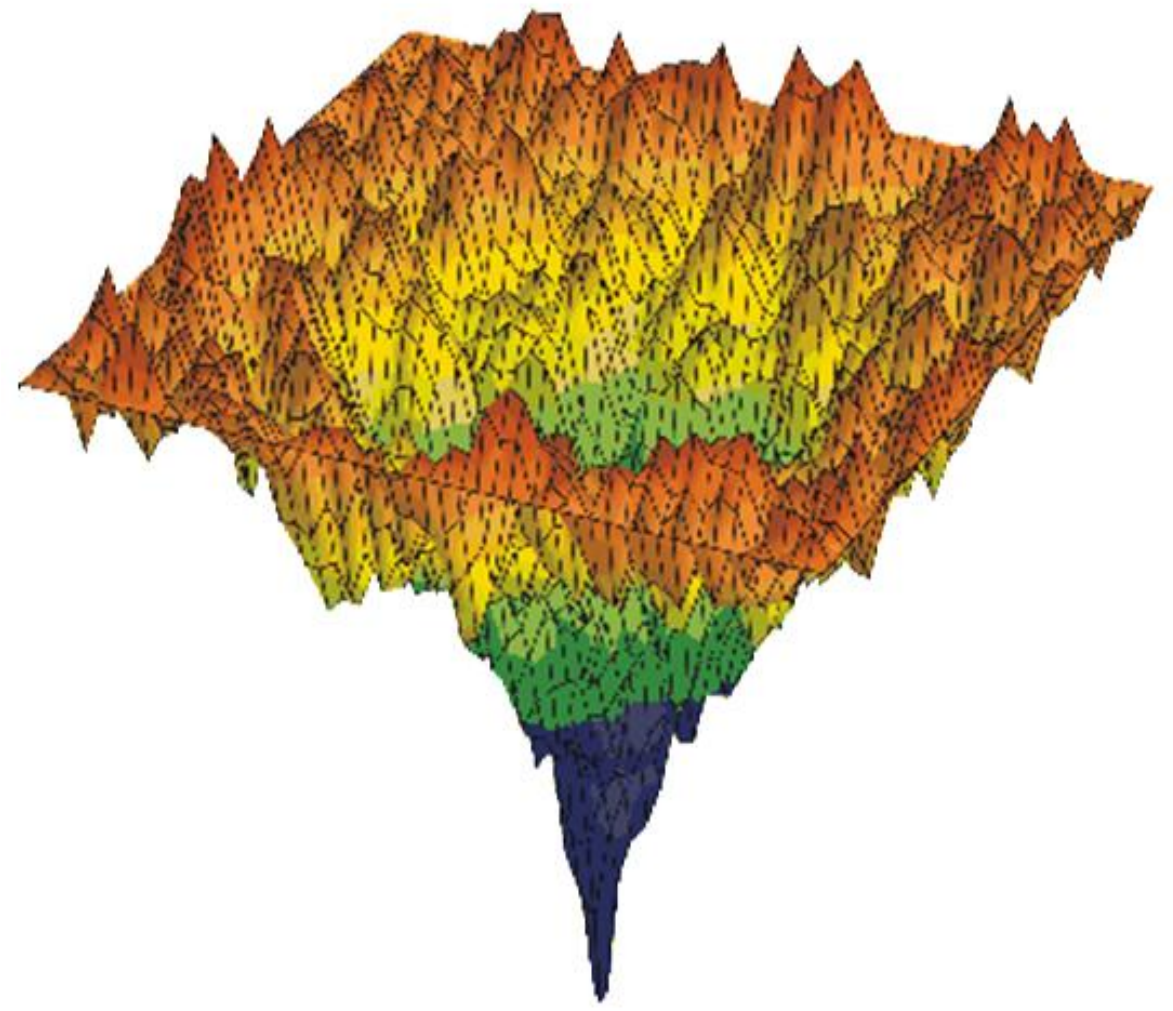

Figure 1.2 The binding energy landscape theory. Protein-ligand binding has a rugged funnel-like free energy landscape.

By comparing the apo crystal structures of same proteins, Najmanovich et al. [162] showed that some rotameric changes exist near the binding sites. Chou and Bax [163] supported this observation with NMR structures. A study of the binding cavity of the apo form of the T4 lysozyme revealed that the helix F, found around the binding cavity, could undergo displacements of 1.5-2.5 $\AA$ with minimum free energy cost [164]. Zhang et al. [165], also showed that other forms of displacement occur in T4 lysozyme as well. There 
is evidence from crystal structures that support the claim that there are multiple protein conformations resulting from domain motions [166]. Many other studies support the idea of multiple protein receptor conformations [167-169].

Among the challenges of protein-ligand docking, poor treatment of the plasticity of the protein receptor (receptor reorganization) [110] raises much concern. Over the years, several research efforts have focused on the development of molecular docking algorithms to enhance the sampling of the flexibility of the protein receptor [153-156, 158]. Unfortunately, however, the strategies adopted by these algorithms only account for slight side-chain motions, with the consideration of backbone plasticity still in an early developmental stage [170]. Currently, the common strategies for incorporating some level of protein receptor plasticity into molecular docking include soft docking, sampling of side chain flexibility, multiple receptor conformation (MRC) docking, and other in silico techniques.

\section{Soft docking approach}

Soft docking involves the use of implicit models [60] that relaxes the criterion for steric fit. It is a way of avoiding the sampling of MRCs by permitting some steric clashes between the receptor and ligand. A typical example of this approach is decreasing the steepness of the repulsive $\left(\mathrm{r}^{-12}\right)$ term of the Leonard-Jones (L-J) potential [171], as illustrated in Figure 1.3, to allow ligands of varying shapes to be docked onto the protein [172]. A soft function creates room for some degree of overlap between the protein receptor and the ligand while allowing minor exploration of the plasticity of the receptor. This approach has been applied in protein-protein [173] and protein-ligand [174-176] 
docking. Ferrari et al. [177] compared the soft docking and hard docking (sampling of MRCs) approaches and arrived at a conclusion that the soft scoring function was better at recognizing known ligands when only one receptor conformation was used, and worse when MRCs were used. The advantages of the soft docking approach over the explicit MRCs sampling strategy reside in its speed and low computational cost. It is, however, challenged by the fact that it accounts for only small structural changes, which may still restrict the binding of some true binders.

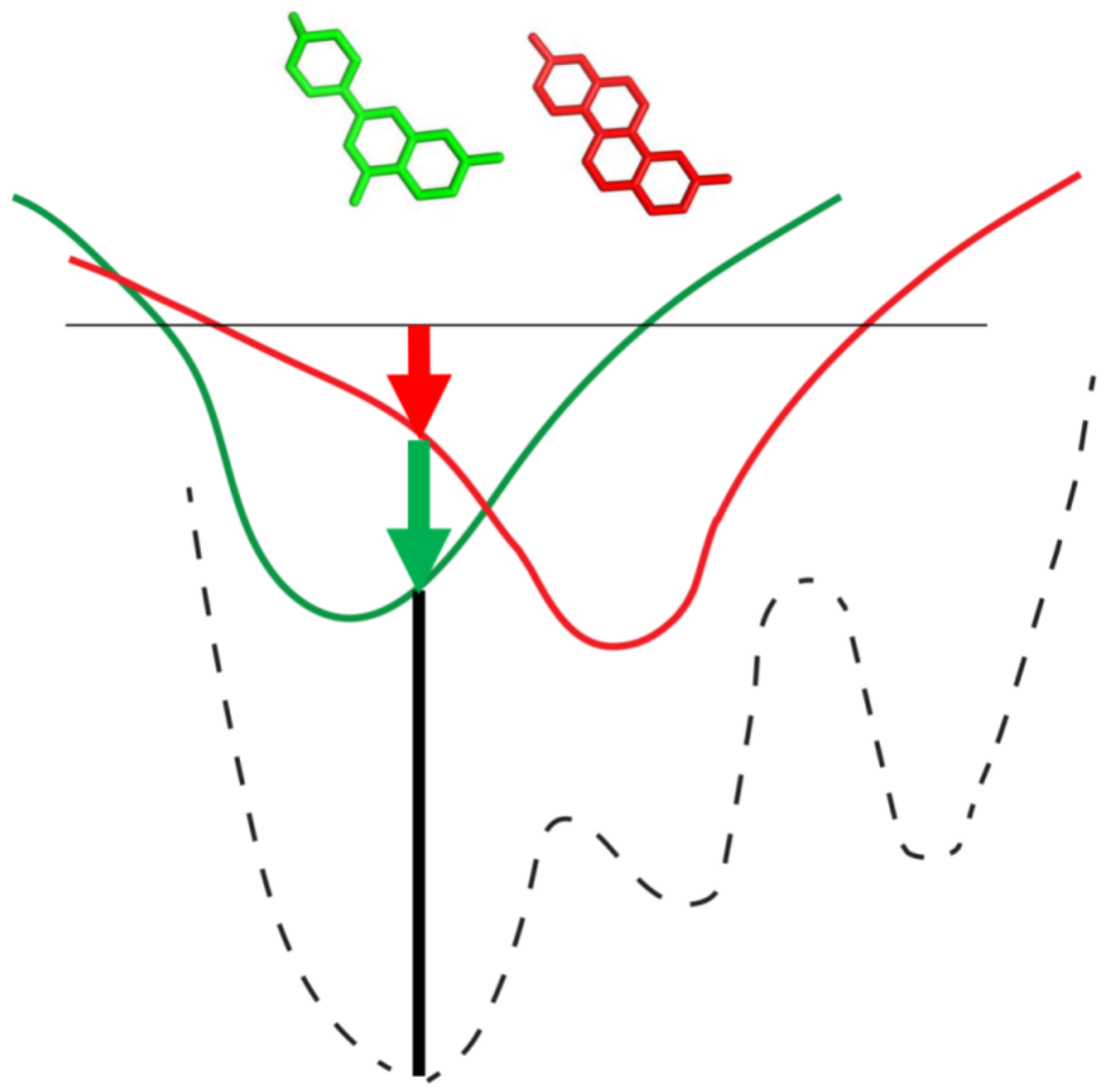

Figure 1.3 Illustration of soft docking approach. The black line indicates a hypothetical protein landscape. Green and red lines represent the protein-ligand interaction energy functions for two different ligands shown in corresponding colors, with arrows representing the computed binding energy in each approximation. Softening the L-J potential allow ligands of different shapes to be docked.(Modified and reprinted from reference [172]) with permission from John Wiley and Sons) 


\section{Sampling of side chain flexibility}

Most docking tools that account for the flexibility of the entire length of the side chains of the protein receptor only permit the torsional rotation of each flexible side chain around one or more of its cyclic bonds during docking. Bond lengths and angles are held fixed while rotations around the bonds account almost fully for the orientations of the side chains in the 3D space.

A recent approach for exploring side chain flexibility involves the use of a rotamer library to generate possible conformations of the side chains of the protein receptor [178-181]. The docked system is then subjected to unrestricted energy optimization to sample the low energy conformations of the ligand and the side chains of the receptor. It is worth noting that most of the applications that employ energy optimization do not include the entire protein. Instead, the local side chains and in some cases local backbones with respect to the binding site are considered. Although this approach is considered to be robust than soft docking in accounting for the plasticity of the receptor, it is limited by the fact that it does not take into consideration the effect of large conformational changes of the receptor.

\section{Multiple receptor conformation docking}

MRC docking incorporates the notion that a protein receptor can assume different conformations (conformational polymorphism) into molecular docking. The strategy assumes that the protein receptor is characterized by accessible isomeric configurational states with close energy. Thus docking onto MRCs allows one to take advantage of the full flexibility of a protein receptor [182] and to explore the local minima without taking the free energy variation into account [172]. 
In the MRC docking technique, ligands are docked onto multiple structures of the protein receptor. Experimental structures generated by X-ray crystallography, NMR, and cryoEM or computational structures generated by homology modeling and simulations are often used. One common approach for implementing MRC docking is referred to as ensemble docking [183]. Figure 1.4 summarizes the various forms of the ensemble docking approach. Figure 1.4A illustrates the fastest ensemble-based docking technique, which involves the use of an averaged structure obtained from several conformations of the protein $[176,183]$. To explore the ensemble in a detailed manner, the ligands are docked onto superimposed multiple structures of the receptor in another technique as shown in Figure 1.4B [183]. The docked solutions are then optimized to find the best scoring fit between a ligand and a conformation. In another method, ligands are docked separately onto each conformation of the receptor as illustrated in Figure 1.4C [184]. The poses are then refined in the flexible pockets and rescored (refining and rescoring are skipped by most protocols) followed by the selection of the best scoring pose(s).

Arguably, docking separately onto each conformation of the receptor provides a more robust means to sample the conformational space. Although MRC docking is widely accepted as the best method to account for the plasticity of the protein receptor, it is limited by the fact that the number of false positives it introduces into the screening process increases with the number of conformations $[172,185,186]$. 


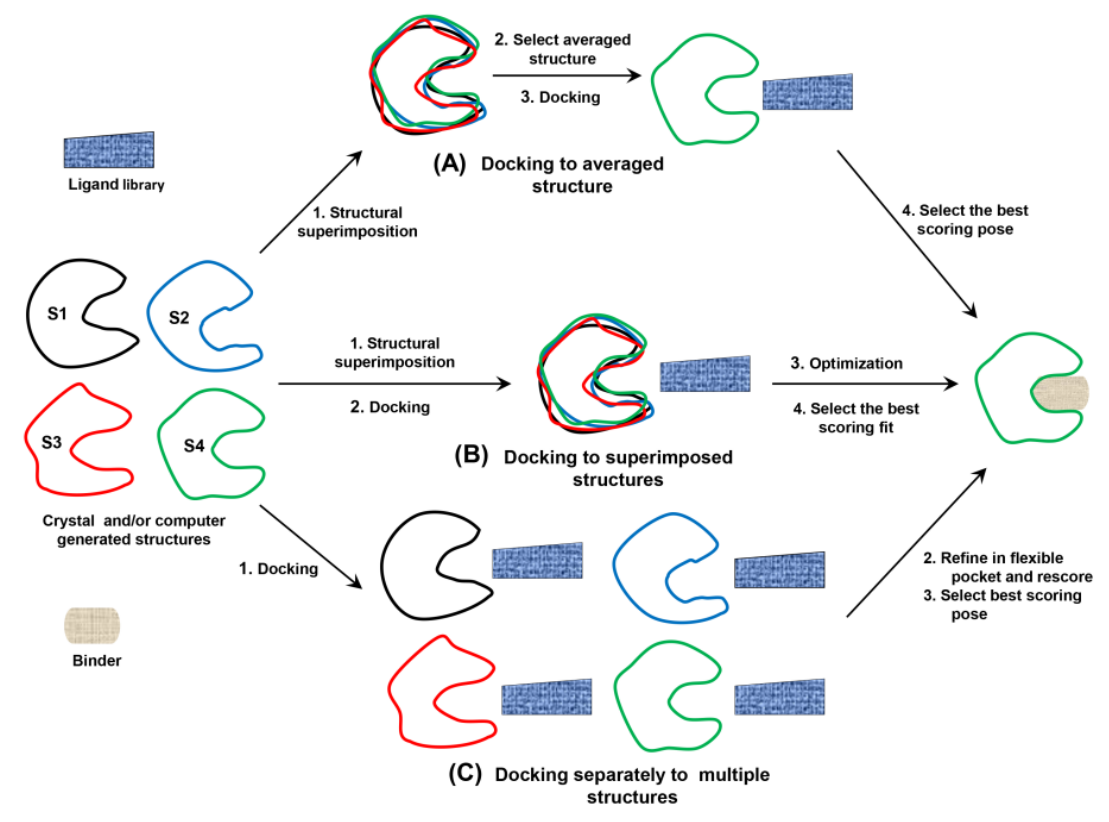

Figure 1.4 A graphical representation of multiple receptor docking strategies. (A) Illustration of averaged protein structure method. (B) Illustration of the superimposed structures method. (C) Illustration of separate multiple receptors method.

\section{In silico techniques}

The use of other computational techniques as a means to incorporate protein-receptor flexibility into protein-ligand docking is rapidly gaining popularity. These techniques could be applied at the pre-docking or post-docking stages. Prior to docking, other computational strategies could be used to generate different conformations of the binding site for ensemble docking. After docking, in silico techniques could also be used to explore additional conformational details of the protein-ligand complex or improve a ligand's pose. Although there are several computational methods such as simulated annealing, MC simulation, low-frequency normal modes, replica-exchange MD (REMD) simulations, and metadynamics for incorporating receptor flexibility into molecular docking, MD simulation is commonly used. 


\section{Molecular dynamics simulation}

MD simulations revolve around the Newton's 2nd law of motion. It involves finding a step-by-step numerical solution to the classical equations of motion of the atoms of a particular system. MD simulation is a powerful tool that bridges the gap between events happening in the microscopic and macroscopic time scales as well as between theory and experiment [187] (Figure 1.5). The technique is used to study complex biological processes such as conformational changes, protein/nucleic acid stability, protein folding, ion transport, and molecular recognition. It is also a useful tool in drug design and the determination of chemical and macromolecular structure by X-ray crystallography and NMR [188, 189]. In drug discovery, MD simulation is an effective means for exploring the plasticity of a receptor, refining receptor-ligand interactions, and predicting drug mechanisms.
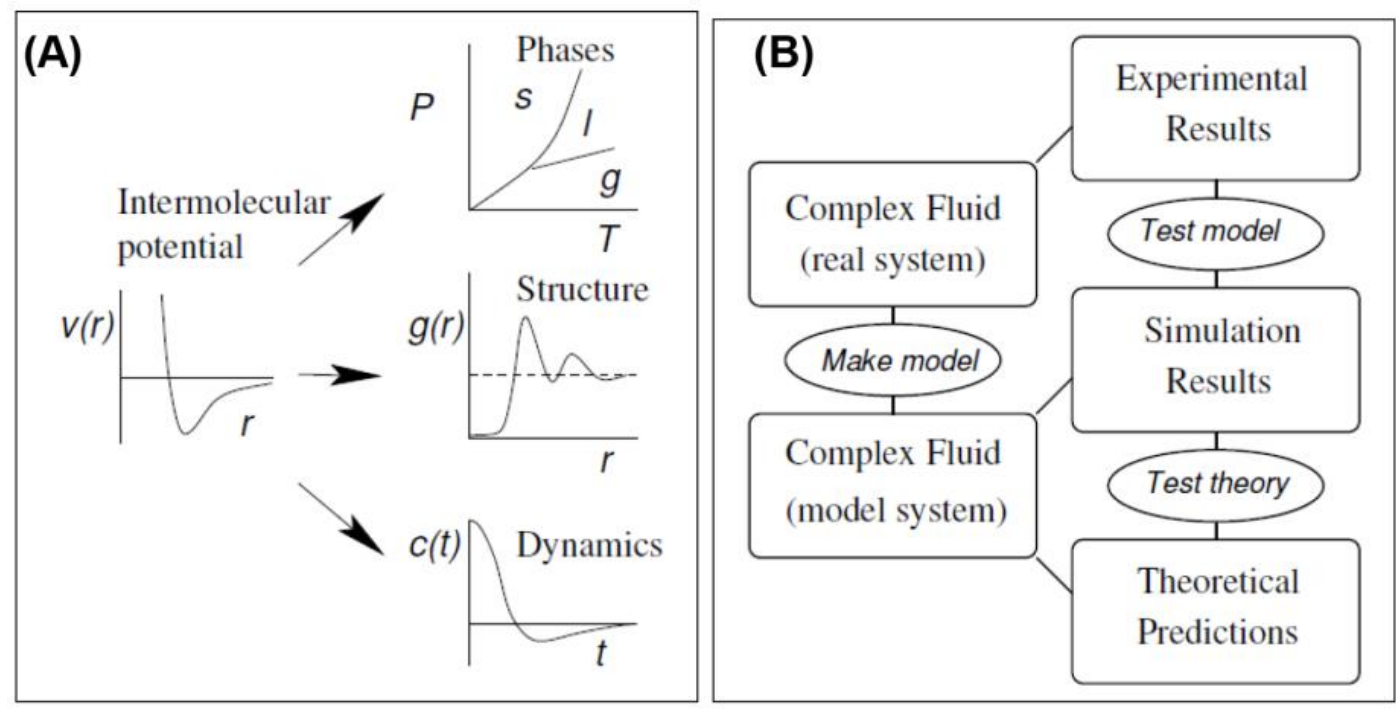

Figure 1.5 Molecular dynamics simulation is a bridge. (A) Between microscopic and macroscopic. (B) Between theory and experiment. (Figure is adapted from Ref. [187]) 
Primarily, MD simulations generate information at the microscopic level. Thus in MD simulations, the macroscopic properties of a system are explored through the microscopic simulations of its atoms. Atomic positions and velocities are the information generated at the microscopic level. Statistical mechanics is then used to convert the microscopic information into macroscopic observables such as heat capacities, energy, and pressure. While experimental observables are normally ensemble averages, properties analyzed by MD simulations are time averages. To resolve this dilemma, the ergodic hypothesis of statistical mechanics is applied. The ergodic hypothesis relates information obtained from MD simulations to experiment via the assumption that the time average equals the ensemble average. For the ergodic hypothesis to be satisfied, it is required that the simulation is long enough to sample conformations that represent all microstates of the system. One of the limitations of MD simulations is the possibility of inadequate sampling as a consequence of the system's inability to cross energy barriers.

Several efforts have been made to use MD simulations to assist molecular docking. MD simulations are commonly used to generate multiple structures of a protein receptor for docking. The MD structures are normally extracted from a trajectory of the apo [190] or the holo [184] form of the receptor. While some argue that extracting structures from the holo form of the receptor is better because it guarantees the conservation of good cavity volume, others think that extracting the structures from the apo form is better because it allows the flexibility of the receptor to be adequately explored and does not lead to the introduction of a form of bias against different classes of molecules. Whichever way they 
are generated, studies show that MD structures are as good as experimental structures for SBVS [191-194].

In addition, MD simulation is also used as a post-docking application to refine the protein-ligand interactions predicted by docking algorithms [195-199]. In classical molecular docking, the ligand can undergo large conformational changes. Although this phenomenon could allow the ligand to cross the energy barriers of the potential energy surface, it could lead to a substantial sampling of unphysical conformations of the proteinligand complex. MD simulation is a good tool for the refinement of such unphysical structures because motion in an MD system is guided by its physical forces. Also, MD simulations of docking complexes in explicit solvent may offer the opportunity to consider the effects of solvent on the interaction between the protein and ligand.

Furthermore, targeted MD simulations [200] can be used to directly dock a ligand onto the binding site. In a study, Samsonov et al [201] developed the dynamic molecular docking (DMD) approach to address the challenges associated with treating flexibility and solvation in molecular docking. The ligand and receptor are placed at a distance apart. A distance-dependent constraint is applied to slowly pull the ligand towards the receptor. Both the ligand and receptor are treated as flexible in explicit solvent. Once the ligand reaches the receptor, the constraint is turned off and a long normal simulation is carried out to sample conformations of the protein-ligand complex. It was shown that the DMD method is much useful when the protein-ligand binding is driven my electrostatic interactions. This approach also faces the challenge of poor conformational sampling by MD algorithms. 


\section{Theory of molecular dynamics simulations}

For a simple atomic system, if a force $f_{i}$ is exerted on an atom $i$ of mass $m_{i}$ to cause an acceleration $a_{i}$, the classical equation of motion is represented by:

$\boldsymbol{f}_{\boldsymbol{i}}=m_{i} \boldsymbol{a}_{\boldsymbol{i}}$

$$
\boldsymbol{a}_{i}=\frac{d^{2} r_{i}}{d t^{2}}
$$

The force may also be expressed as the slope of the potential energy (U) as:

$\boldsymbol{f}_{\boldsymbol{i}}=-\Delta_{i} U=-\frac{d}{d r_{i}} U$

Combining (4), (5) and (6) gives:

$$
-\frac{d U}{d r_{i}}=m_{i} \frac{d^{2} r_{i}}{d t^{2}}
$$

For a system made up of $\mathrm{N}$ particles, $\mathrm{U}$ is dependent on the cartesian coordinates $(\mathrm{x}, \mathrm{y}$, and $\mathrm{z}$ ) of all the atoms in the system as:

$U=\sum_{i=1}^{N} f_{i}(\mathrm{x}, \mathrm{y}, \mathrm{z})$

$\mathrm{U}$ can also be defined as a function of the sum of energy terms such as bond vibration, bond angles, torsion, van der Waals interactions and electrostatic interactions. Thus,

$$
\begin{aligned}
& U=E_{\text {bond }}+E_{\text {angle }}+E_{\text {torsion }}+E_{\text {vdW }}+E_{\text {ele }} \\
& E_{\text {bond }}=\sum \frac{k_{b}}{2}\left(l-l_{0}\right)^{2}
\end{aligned}
$$


$E_{\text {angle }}=\sum \frac{k_{\theta}}{2}\left(\theta-\theta_{0}\right)^{2}$

$E_{\text {torsion }}=\sum \frac{k_{\emptyset}}{2}\left[1+\cos N\left(\varnothing-\emptyset_{0}\right)\right]$

$E_{v d W}=\sum 4 \epsilon\left[(\sigma / r)^{12}-(\sigma / r)^{6}\right]$

$E_{\text {ele }}=\sum \frac{q_{1} q_{2}}{4 \pi \varepsilon_{0} r}$

$E_{\text {bond }}$ is the energy contribution that results from the variation of a bond length from its equilibrium value. A harmonic potential describes this energy. $E_{\text {angle }}$ is the energy contribution resulting from the variation of a bond angle from its equilibrium value. $E_{\text {torsion }}$ is the energy contribution that emanates from variation of a dihedral angle from its natural value. $E_{v d W}$ is the energy contribution from non-bonded van der Waals interactions. It is composed of the attractive and the repulsive terms. $E_{\text {ele }}$ arises from electrostatic interactions between charged atoms.

Thus once the magnitude of the force on each atom in the system is known, the corresponding accelerations can be determined. By integrating the equations of motion, a trajectory which defines the microscopic properties of the atoms of the system as they change with time is generated. The time-averaged properties of the system can then be deduced from the trajectory. The method is deterministic in the sense that by knowing the position and velocity of each atom, the state of the system at any point in time in the past or future can be predicted. 


\subsubsection{Lead optimization}

Once a hit compound is discovered in the lead discovery phase, the next stage in the drug discovery process is lead optimization. Lead optimization involves interactive modifications and characterization of a hit compound to improve its affinity, selectivity, potency, metabolic stability, bioavailability, and other pharmacological properties [202, 203]. Only the hit compound that meets all these requirements is passed on to the drug development phase prior to clinical trials.

Some of the key steps involved in lead optimization include (a) Pharmacophore identification: it involves identification of the relevant groups on the hit compound that are responsible for its interaction with the biological target [204-206]. These groups are likely to be responsible for biological activity $[205,206]$ and as much as possible should be kept or improved, unless they are known to be the cause of some other undesirable properties of the compound, (b) Modification of functional groups: functional groups on small molecules are usually responsible for some desirable or undesirable pharmacological properties. Thus functional groups could be modified to activate or deactivate a particular biological or pharmacological effect of the hit compound, and (c) Structure-activity relationship (SAR): it is based on the idea that the structure of a chemical compound contains fingerprints that influence biological activity [207-209]. Several homologs of the hit compound are designed, synthesized, characterized, and tested to determine the structure with the best pharmacological properties. 


\subsection{Protein receptors used in this study}

\subsubsection{Influenza A nucleoprotein}

The influenza A nucleoprotein (NP), is a flu protein whose main role in the virus' life cycle is to bind to the segmented RNA polymerase and its subunits (PA, PB1 and PB2) to form ribonucleoproteins (RNPs), which are then transported into the host cell's nucleus where viral genome replication takes place. The crystallographic structures of the monomeric and trimeric forms of NP are shown in Figure 1.6. The protein is characterized by a head domain consisting of a tail-loop (T-loop) and a body domain within which are found the T-loop binding pocket and RNA binding groove [210] (Figure 1.6A). NPs, which are synthesized as monomers, aggregate into trimers before binding to RNA. The oligomerization process, a prerequisite for RNA binding, is aided by the insertion of the T-loop of one monomer into the T-loop binding pocket of a neighboring monomer [210] (Figure 1.6B).

NP is expressed abundantly during infection and is more conserved among influenza viruses than other viral proteins such as hemagglutinin and neuraminidase [211-213]. Thus molecules that could inhibit NP oligomerization and/or RNA binding could be potential antiviral drug candidates. Studies have identified the T-loop binding pocket [210, 214], RNA binding site [215], and a nucleozin binding site [216] of NP as targets for antiviral drug development. 
(A)

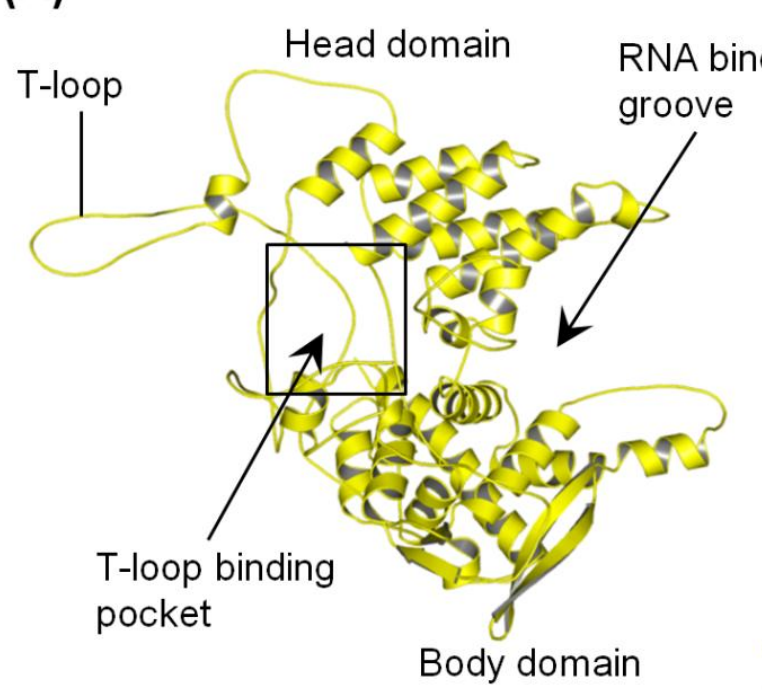

(B)

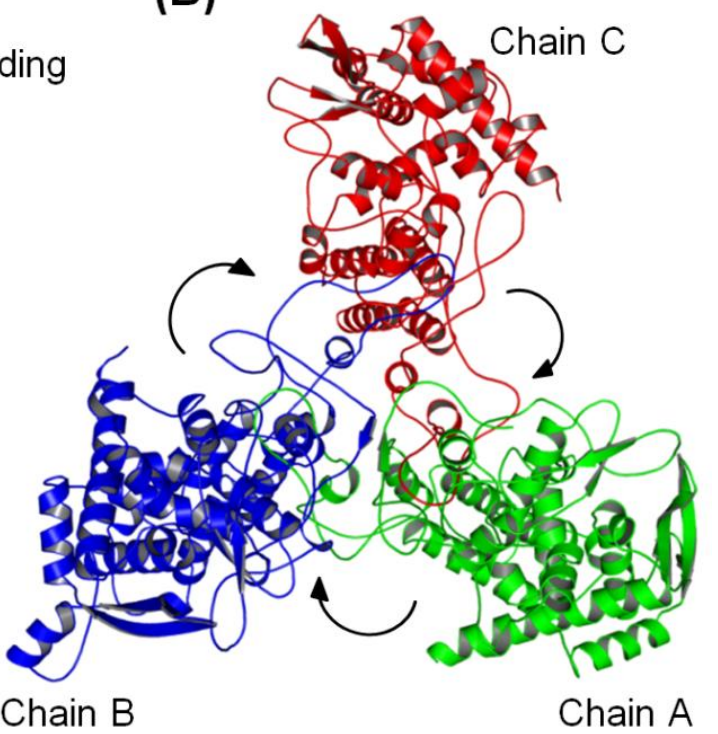

Figure 1.6 Cartoon representations of the structure of influenza A Nucleoprotein. (A) Monomeric structure of NP showing the head and body domains, T-loop, T-loop binding pocket and the RNA binding groove. (B) Trimeric structure of NP illustrating how NP-NP interaction occurs prior to RNA binding.

\subsubsection{Inhibitors of influenza A nucleoprotein}

Figure 1.7 shows some known inhibitors of NP. 2-[(3,4-dichlorophenyl)amino]-N-[3(morpholin-4-yl)propyl]-1,3-thiazole-4-carboxamide (DAMPTC) is believed to inhibit the oligomerization of NP into trimmers by binding to the T-loop binding pocket of one monomer to obstruct the insertion of the T-loop of a neighboring monomer [214]. It has been shown in an antiviral assay involving MDCK cells that DAMPTC can inhibit A/WSN/33 (H1N1) with an $\mathrm{IC}_{50}$ value of $2.7 \mathrm{Mm}[214]$.

It has been suggested that F66 and naproxen target the arginine-rich RNA binding groove of NP and could be inhibitors of RNA binding [215, 217]. F66 has been shown to be 
effective against a variety of influenza A strains in vitro and in vivo, but less effective against influenza B strains [215]. Naproxen is an anti-inflammatory drug and an inhibitor of inducible cyclooxygenase type $2(\mathrm{COX}-2)$. A recent study showed that naproxen can inhibit NP-RNA association and is effective against influenza $\mathrm{A}$ in vitro and in vivo [217].

Nucleozin inhibits NP by functioning as an antagonist of its accumulation in the nucleus of the virus [216]. A number of studies have discovered nucleozin analogues with a similar mode of action [218-220]. This class of NP inhibitors induces the formation of higher-order NP oligomers, which are unable to enter the nucleus of the virus for genome replication to take place [220]. It has been demonstrated that nucleozin and its analogs can inhibit the replication of influenza A virus in vitro and in mice [216, 218-220].<smiles>O=C(NCCCN1CCOCC1)c1nc(Nc2ccc(Cl)c(Cl)c2)cs1</smiles><smiles>COc1ccc(/C=N/NC(=O)CSc2nnc(-c3ccc(Cl)cc3)n2-c2ccc(C)cc2)cc1Br</smiles><smiles>COc1ccc2cc([C@@H](C)C(=O)O)ccc2c1</smiles><smiles>CCOC(=O)NCc1onc(-c2ccccc2)c1C(=O)N1CCN(c2ccc([N+](=O)[O-])cc2Cl)CC1</smiles>

Figure 1.7 Inhibitors of influenza nucleoprotein. 


\subsubsection{Bacterial actin MreB}

The MreB protein is encoded by gene B (mreB) of the mre (murein cluster e) genes. Other genes that belong to this cluster are $\mathrm{mreC}$ and $\mathrm{mreD}$. MreB is common in prokaryotes with complex nonspherical shapes but absent in the spherical ones [221]. It is present and proves to be vital in most bacteria, including Bacillus (B) subtilis [221, 222], Escherichia (E) coli [223], Caulobacter (C) crescentus [224], Thermotoga (T) maritima [225], Rhodobacter $(R)$ sphaeroides [226], Salmonella $(S)$ Typhimurium [227, 228], Streptomyces (S)coelicolor [229, 230], and Pseudomonas (P) aeruginosa [231].

Until MreB was discovered in prokaryotes [232], it was believed that actin was only present in eukaryotes. Although the primary structure of MreB compares poorly with that of actin, it is regarded as a prokaryotic version of actin by virtue of revelations that the tertiary structure of monomeric $\mathrm{MreB}$ is closely similar to the tertiary structure of monomeric eukaryotic actin [233] and that it could aggregate into polymers in vitro [233$235]$ and in vivo [221, 236]. Mutations in the mreB gene transform rod-shaped cells to spherical cells, suggesting that the MreB plays a role in cell shape formation [237].

Although MreB performs many equivalent roles of actin in prokaryotes, it has been shown that its filaments and those of actin are different in structural details [238]. Both actin and MreB filaments are made of two strands, but while the strands of actin are twisted and parallel with each other, those of MreB are straight and antiparallel. Thus the two ends of actin filament can be clearly differentiated from each other but those of MreB are not distinguishable [238]. The monomeric and filamentous crystallographic structures of $C$. crescentus MreB are shown in Figure 1.8. 

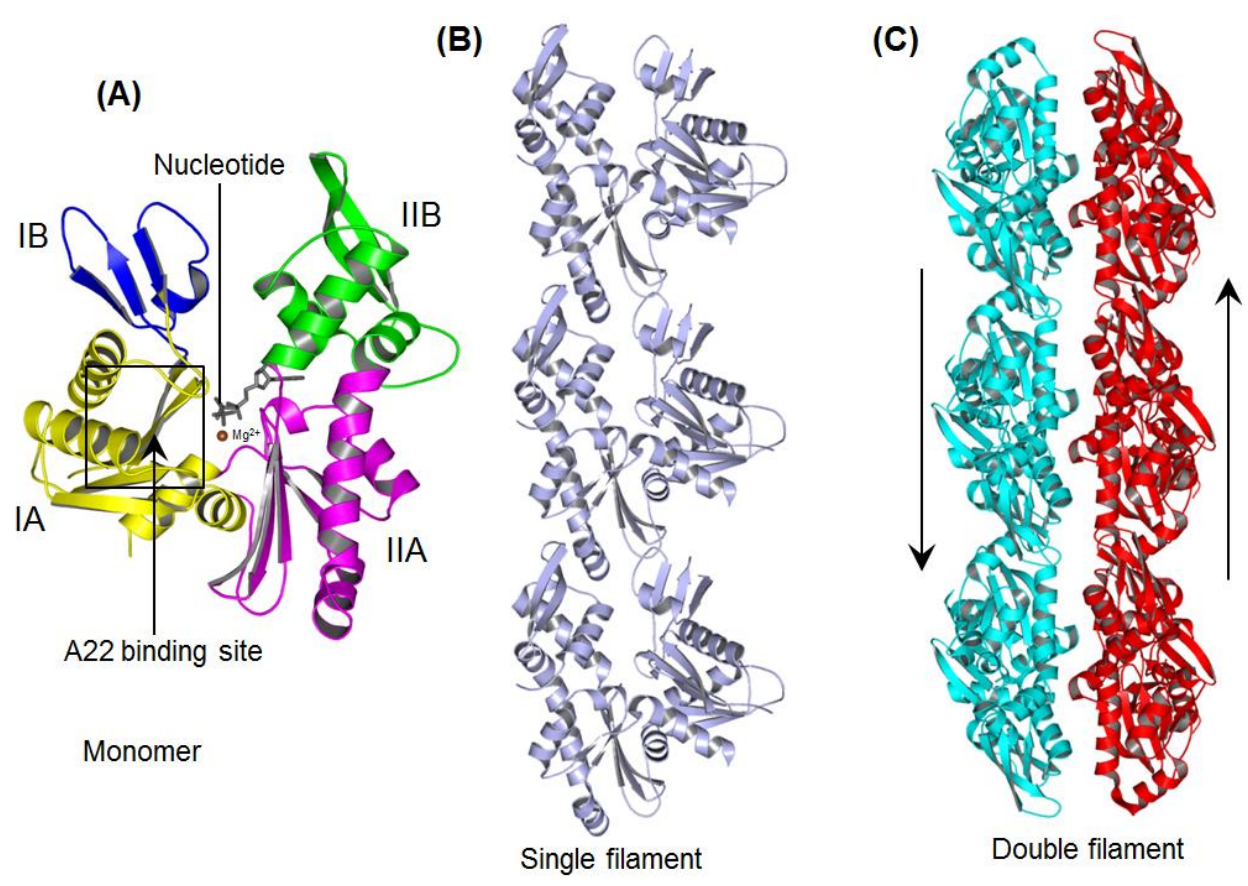

Figure 1.8 Cartoon representations of MreB structures. (A) Monomeric structure of MreB. The nucleotide (NT) and A22 binding sites and the four subdomains of the protein are indicated. (B) The structure of a single filament of MreB. (C) The structure of a double filament of MreB.

It has been established that MreB plays a vital role in the establishment and maintenance of cell shape [221, 232], cell division [239], chromosome segregation [240-243], cell wall morphogenesis [244], and cell polarity [245] in many bacteria. This actin homolog, consisting of four subdomains surrounding its nucleotide (NT)-binding pocket (Figure 1.8A), is an ATPase that requires ATP to polymerize into filaments (Figure 1.8B-C), and polymerization is required for cell wall synthesis [233, 246]. Polymerization of MreB induces hydrolysis of ATP, which acts as a timing mechanism to coordinate depolymerization $[225,234,246,247]$. Several studies have suggested that bacterial MreB, which is conserved across most rod-shaped bacteria and absent in eukaryotes, may be targeted for the development of antibiotics [223, 248, 249]. 


\subsubsection{Inhibitors of MreB}

The known inhibitors of MreB are shown in Figure 1.9. S-(3, 4-Dichlorobenzyl) isothiourea (A22) and S-(4-chlorobenzyl) isothiourea (MP265) are structural analogs that disrupt the rod shape [250-252] of bacterial cells by targeting MreB [253]. A22 has been shown to be effective against E. coli, (minimum inhibitory concentration (MIC): $3.13 \mu \mathrm{g} / \mathrm{ml})$ than gram-negative bacteria such as Staphylococcus $(S)$ aureus, B. subtilis, Pseudomonas $(P)$ putida and S. typhimurium [250-252]. Crystal structures show that both A22 and MP265 bind adjacent to NT in the putative ATP-binding pocket of MreB. However, the precise mechanism of these inhibitors is yet to be determined.

CBR-4830 is an indole-class compound that inhibits the growth of an efflux-compromised P. aeruginosa (MIC: $5 \mu \mathrm{g} / \mathrm{ml}$ ) by inducing coccoid shape [231]. It is believed to target MreB by interacting with the ATP-binding pocket [231]. The exact binding site and mechanism of CBR-4830 are yet to be elucidated.
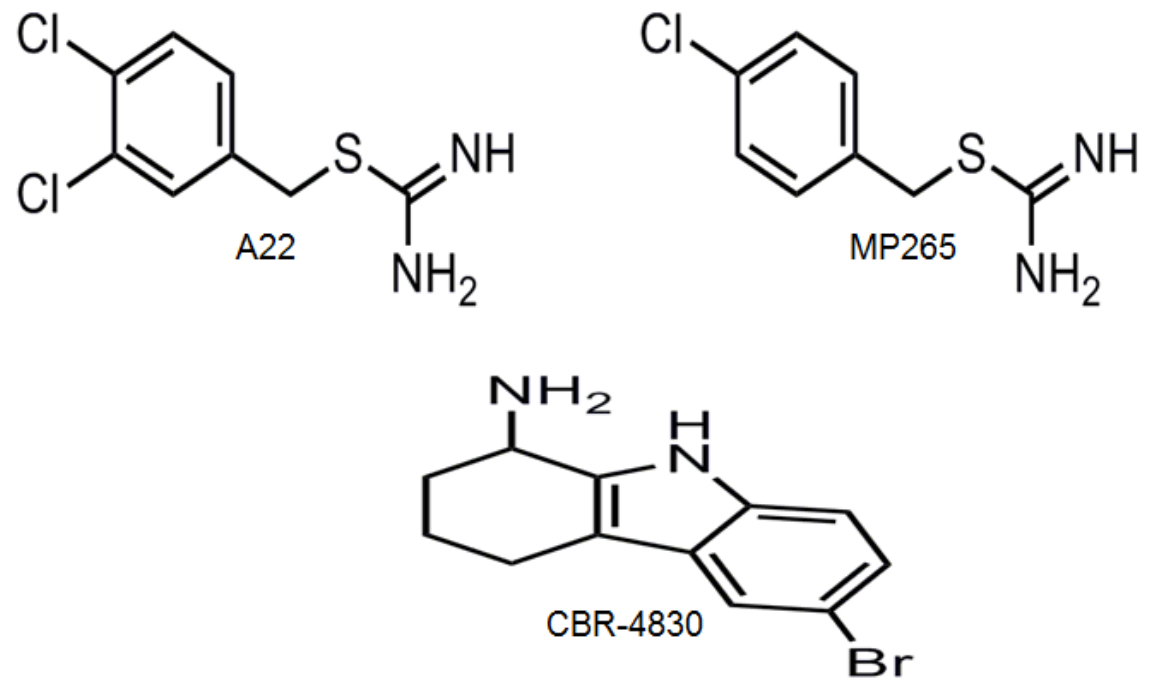

Figure 1.9 Inhibitors of MreB. 


\subsection{Aims and Objectives}

The plasticity of protein is a naturally occurring process that can greatly influence the molecular recognition process [254], and thus affects the binding mode prediction of ligands [176, 255]. Substrate binding, catalysis, and product release are all processes that could be affected by protein flexibility [256, 257]. Therefore, it is essential to extensively consider the flexibility of the protein receptor and protein-ligand complex in the drug discovery process.

The MRC docking approach that involves docking separately onto each distinct conformation of the receptor is widely accepted as the most robust means for accounting for the plasticity of the protein receptor in SBVS. This strategy, however, leads to an increase in the number of false positives in the predicted drug candidates $[172,185,186]$. Unfortunately, the current MRC docking protocol in which the best scoring pose(s) from each receptor conformation is/are selected do not address the challenge posed by the false positives. The main aim of this study was to exploit the plasticity of the protein receptor to improve the accuracy of SBVS. In the first place, it was sought to devise a strategy to reduce the false positives encountered in SBVS when receptor plasticity is considered and to identify true binders. To accomplish this goal, distinctive binding site conformations of NP and the bacterial actin MreB were generated for MRC docking exercises. A ligand selection criterion was devised to exclude false positives and identify true binders. To validate the selection strategy, crystallographic studies to ascertain whether the hits identified by the criterion can bind to MreB was carried out. Finally, to gain insight into the dynamics of the active of MreB, understand the mechanism of A22 and obtain 
information that could be channeled into the design and development of more effective inhibitors of MreB, MD simulations of MreB in complex with A22 in the presence of different NTs were carried out. 


\section{Materials and Methods}

\subsection{Materials}

The crystal structures of (i) NP (PDB ID: 2IQH [210]) and (ii) C. crescentus MreB in complex with AMPPNP, $\mathrm{Mg}^{2+}$, and MP265 (PDB ID: 4CZK [238]); and with ADP, $\mathrm{Mg}^{2+}$, and A22 (PDB ID: 4CZG [238], ADP-A22-bound state) were downloaded from the RCSB PDB. The sdf structure files of the Otava primeScreen1 diversity (OP1D) library and the Maybridge fragment (MF) library were downloaded from the websites of Otava Chemicals and Maybridge Chemical Company respectively. The OP1D library was selected for docking against the T-loop binding pocket and the RNA binding site of NP. The A22 binding site of MreB was the target of the MF library. The molecules A1, B2, C3, D4, E5, F6, G7, H8, I9, J10, and K11 were bought from Maybridge Chemical Company. The chemical names of these molecules are provided in Table A.2 in Appendix A. The X-ray crystallographic experiments to validate the ligand selection strategy described in this thesis were carried out through a collaboration with A/Prof. Robert C. Robinson and Dr. Shimin Jiang of the Institute of Molecular and Cell Biology (IMCB, A*STAR). They provided the crystal structures reported in Chapter 4.

\subsection{Methods}

\subsubsection{Preparation of influenza A nucleoprotein}

Chains B and C of the homotrimeric crystal structure of NP were deleted to generate a monomeric structure consisting of chain A. Seventy-three residues missing were replaced using the SWISS-MODEL package [258]. The missing residues include Arg8, Ser9, 
Tyr10, Glu11, Gln12, Met13, Glu14, Thr15, Asp16, Gly17, Glu18, Arg19, Gln20, Glu73, Arg74, Arg75, Asn76, Lys77, Tyr78, Leu79, Glu80, Glu81, His82, Pro83, Ser84, Ala85,

Gly86, Lys87, Asp88, Pro89, Lys90, Lys91, Asp203, Arg204, Asn205, Phe206, Trp207, Arg208, Gly209, Glu210, Asn211, Gly212, Asn397, Gln398, Gln399, Arg400, Ala401,

Phe429, Thr430, Gly431, Asn432, Thr433, Glu434, Gly435, Arg436, Thr437, Gly490, Asp491, Asn492, Ala493, Glu494, Glu495, Tyr496, Asp497, Asn498, Leu499, Glu500, His501, His502, His503, His504, His505, and His506. This monomeric form of NP was used in this study.NP-DAMPTC and NP-molecule A complexes (holo forms) were generated by docking with the GOLD software [48, 53, 129]. Details of the docking procedure are the same as described later in this chapter.

\subsubsection{Preparation of MreB structures}

Eight missing residues, which constitute two non-terminal loops, were added to each of the MreB structures using the SWISS-MODEL package [258]. These include Val44, Gly45, Gly46, and Arg47 for one loop, and Ala226, Asp227, Gly228, and Glu229 for the other. A22 has two chlorine atoms attached to the carbon atoms at positions 3 and 4 of its benzene ring, while MP265 has only one chlorine atom attached to the carbon atom at position 4 (See Figure 1.9). Crystal structures of both molecules in complex with MreB [238] show that their modes of binding are similar. Therefore, 4CZK (AMPPNP-MP265bound state) was modified as follows: (i) AMPPNP was modified to ATP, while MP265 was modified to A22 to form the ATP-A22-bound state. (ii) MP265 was modified to A22 to form the AMPPNP-A22-bound state. (iii) AMPPNP was removed, while MP265 was modified to A22 to form the NT-free state. (iv) AMPPNP was modified to ATP, while 
MP265 was removed to form the ATP-bound state. The ADP- $\mathrm{P}_{\mathrm{i}}-\mathrm{A} 22$-bound state was generated from an MD structure obtained from the simulation of the ATP-A22-bound state. The $\mathrm{P}_{\mathrm{i}}$ was constructed using Accelrys Discovery studio (DS) [128]. Deprotonated forms of ATP, AMPPNP, ADP, and $\mathrm{P}_{\mathrm{i}}$ were used throughout this study.

\subsubsection{Molecular dynamics simulations}

\subsubsection{Simulation of drug-free structures}

In the first batch of simulations, the prepared drug-free structures of NP and MreB (ATPbound, without A22) were used as initial structures for normal MD simulations. The simulations were performed with Gromacs 4.5.4 [259], Amber 99SB force field [260], a time step of $0.002 \mathrm{ps}$, the Berendsen barostat for pressure, and the V-rescaling thermostat [261] for temperature coupling. The Chimera software [262] was used to determine the protonation states of the histidine residues of both proteins. The MreB structure contained ATP, whose topology was built as described in the next section. The topologies of both proteins were built with the pdb2gmx utility in Gromacs.

In each case, the protein was centered $10 \AA$ from the edges of a cubic box and solvated in an explicit TIP3P water model [263]. The system was neutralized electrostatically by adding the appropriate number of ions, and $0.1 \mathrm{M} \mathrm{NaCl}$ was added to maintain physiological ionic strength. Energy minimization of the system was carried out using the steepest descent algorithm with an energy step of 0.05 and a maximum force threshold of $1.0 \mathrm{~kJ} / \mathrm{mol}$. The Particle Mesh Ewald (PME) algorithm [264] was applied for the treatment of long-range electrostatics with $10 \AA$ for real-space cutoffs. The van der Waals interactions were cut off at $14 \AA$. At $300 \mathrm{~K}$, the system was equilibrated using NPT 
ensemble over $5 \mathrm{~ns}$. The crystal structure of each protein was used as the seed structure from which five independent MD trajectories of $20 \mathrm{~ns}$ each were generated with randomly assigned initial velocities. To adequately explore the phase space of each protein and to sample as many conformations as possible, the final configurations of the five simulations were taken as seed structures for producing another batch of five independent $20 \mathrm{~ns}$ trajectories with randomly chosen initial velocities. All $10 \mathrm{MD}$ trajectories for each protein were then merged into a 200 ns combined trajectory.

In the second batch of simulations of drug-free structures, the ATP-bound and ADP-P $\mathrm{i}^{-}$ bound states of MreB in the absence of A22 were each simulated for 100 ns with three independent repeats. The topology of each NT was built as described in the next section. All other steps were the same as described above. Table 2.1 shows the summary of the drug-free simulation systems.

Table 2.1 Table summary of MD simulations of drug-free structures.

\begin{tabular}{|c|c|c|c|c|}
\hline \multicolumn{5}{|c|}{ NP structure } \\
\hline System & Structure & Time (ns) & Repeats & Net (ns) \\
\hline 1 & NP & 20 & 10 & 200 \\
\hline \multicolumn{5}{|c|}{ MreB structures } \\
\hline System & Structure & Time (ns) & Repeats & Net (ns) \\
\hline 1 & ATP-bound & 20 & 10 & 200 \\
\hline 2 & ATP-bound & 100 & 3 & \\
\hline 3 & ADP-P $\mathrm{P}_{\mathrm{i}}$-bound & 100 & 3 & \\
\hline
\end{tabular}




\subsubsection{Simulation of drug-bound structures}

The drug-bound structures include NP-DAMPTC, NP-molecule A, MreB-ATP-A22, MreB-AMPPNP-A22, MreB-ADP-A22, MreB-A22 (NT-free), and MreB-ADP-P-A22 complexes. The basic MD protocol was the same as described in the previous section with slight modifications. In each case, the pdb2gmx utility in Gromacs 4.5.4 [265] was used together with Amber99SB force field [260] to build the topology of the protein using the TIP3P water model [263] for solvation. Partial charges of the ligands (DAMPTC, molecule A, A22, AMPPNP, ATP, ADP, and $\mathrm{P}_{\mathrm{i}}$ ) were determined by quantum mechanical calculations performed with Gaussian09 [266] using the R.E.D.-III.4 tool [267]. The antechamber utility [268], which is included in the AMBER10 package [269], was used to assign atom types to the ligands. The topologies of the ligands were then built in the frame of the general amber force field (GAFF).

For each system, a cubic box solvated with an explicit TIP3P water model [263] was used with $10 \AA$ as a minimum distance between the edges of the box and the surface of the protein. The system was neutralized electrostatically by adding the appropriate number of ions, and $0.1 \mathrm{M} \mathrm{NaCl}$ was added to maintain physiological ionic strength. Energy minimization of the system was carried out using the steepest descent algorithm with an energy step size of 0.01 and a maximum force threshold of $10.0 \mathrm{~kJ} / \mathrm{mol}$. The PME algorithm [264] was applied for the treatment of long-range electrostatics with $10 \AA$ for

real-space cutoffs. The van der Waals interactions were cut off at $14 \AA$. With a time step 
of $0.002 \mathrm{ps}$, the Berendsen barostat for pressure and the V-rescaling thermostat [261] for temperature coupling, the system was equilibrated using the isothermal-isobaric NPT ensemble over $5 \mathrm{~ns}$ at $300 \mathrm{~K}$. Continuation of simulations to $100 \mathrm{~ns}$ was performed for each system. Table 2.2 shows the summary of the drug-bound simulation systems.

Table 2.2 Table summary of MD simulations of drug-bound structures.

\begin{tabular}{|c|c|c|c|c|c|c|}
\hline \multicolumn{7}{|c|}{ NP structures } \\
\hline System & State & \multicolumn{2}{|c|}{ Ligand } & \multicolumn{2}{|r|}{ Time (ns) } & Repeats \\
\hline 1 & DAMPTC-bound & \multicolumn{2}{|c|}{ DAMPTC } & \multicolumn{2}{|r|}{10} & 1 \\
\hline 2 & Molecule A-bound & \multicolumn{2}{|c|}{ Molecule A } & \multicolumn{2}{|r|}{10} & 1 \\
\hline \multicolumn{7}{|c|}{ MreB structures } \\
\hline System & State of MreB & Nucleotide & $\mathbf{A 2 2}$ & $\mathbf{M g}^{2+}$ & Time (ns) & Repeats \\
\hline 1 & ATP-A22-bound & ATP & + & + & 100 & 3 \\
\hline 2 & AMPPNP-A22-bound & AMPPNP & + & + & 100 & 3 \\
\hline 3 & ADP-A22-bound & $\mathrm{ADP}$ & + & + & 100 & 3 \\
\hline 4 & Nucleotide-free & - & + & + & 100 & 3 \\
\hline 5 & ADP-P $\mathrm{P}_{\mathrm{i}}-\mathrm{A} 22$-bound & $\mathrm{ADP}+\left(\mathrm{P}_{\mathrm{i}}\right)$ & + & + & 100 & 3 \\
\hline 6 & ADP-P $\mathrm{P}_{\mathrm{i}}$-bound & $\mathrm{ADP}+\left(\mathrm{P}_{\mathrm{i}}\right)$ & - & + & 100 & 3 \\
\hline
\end{tabular}

\subsubsection{Clustering for distinctive structures}

The net $200 \mathrm{~ns}$ trajectory from the MD simulations of the drug-free forms of NP and MreB contained 200,000 frames each. The two trajectories were clustered for representative structures as follows. Two separate clustering exercises were carried out on the NP trajectory for representative structures of the T-loop binding pocket and the RNA binding site. For the T-loop binding pocket, the atoms of twelve selected amino acid residues within the pocket were used as the reference group for structure superimposition 
and clustering for distinctive conformations of the pocket. These residues have been reported by an earlier study to be involved in the stabilization of NP-NP interactions [210]. They include Ser165, Arg267, His272, His334, Ser335, Glu339, Asp340, Ile347, Ile388, Arg389, Ser457, and Phe458. Using the gromos method of g_cluster utility in the Gromacs package [265] and a clustering RMSD cutoff of $0.25 \mathrm{~nm}$, the $200 \mathrm{~ns}$ combined trajectory was grouped into five main clusters. The central structure of each of the five clusters was selected as the representative of the cluster.

For the RNA binding site, the atoms of 13 residues within the proposed binding site of F66 and its derivatives were used as the reference group for structure superimposition and clustering for distinctive conformations. These residues include Arg174, Arg175, Ser176, Gly177, Ala178, Ala179, Gly180, Ala181, Ala182, Val183, Lys184, Met189, and Glu192. Using a clustering RMSD cutoff of $0.18 \mathrm{~nm}$, the $200 \mathrm{~ns}$ combined trajectory was grouped into four main clusters. The central structure of each of the four clusters was selected as the representative of the cluster.

To obtain varying conformations for the A22 binding site of MreB, the atoms of 10 residues that form the binding site were used as the reference group for superimposition and clustering. The residues include Leu17, Gly18, Met74, Ile79, Phe82, Val109, Pro112, Ala115, Ile123, and Glu140. Using a clustering RMSD cutoff of $0.17 \mathrm{~nm}$, three distinct representative structures of the A22 binding site of MreB were selected from the corresponding $200 \mathrm{~ns}$ MreB trajectory. Thus sets of five, four, and three distinctive MD structures of the T-loop binding pocket of NP, the RNA binding site of NP, and the A22 binding site of MreB, respectively, were selected for VS exercises. 


\subsubsection{Docking and in silico screening}

All dockings were performed with the GOLD program [48, 53, 129]. Flexible ligands were docked onto partially flexible and distinct conformations of each receptor. Sets of six, five, and four distinctive structures (the MD generated structures plus the crystal structure of each receptor) of the T-loop binding pocket of NP, the RNA binding site of $\mathrm{NP}$, and the A22 binding site of MreB, respectively, were separately used as receptor models in the docking process. The receptors were prepared with the automatic protein preparation tool in DS [128], during which protons and partial charges were added. All prepared proteins were saved as mol2 files.

The OP1D and MF libraries were both prepared for docking with the automatic ligand preparation tool in DS [128]. Protonation of the molecules was done within a $\mathrm{pH}$ range of 6.5 to 8.5 . In total, 1000 and 30,036 molecules were prepared for the OP1D and the MF libraries respectively. Two molecules, DAMPTC and N-[2-[(3-Fluorophenyl) amino]-2oxoethyl]nonanamide (FAON), whose binding affinities for the T-loop binding pocket of NP have been measured in previous experiments [214] were added to the OP1D library to serve as the high-affinity control (HAC) and the low-affinity control (LAC) respectively, for the T-loop binding pocket. Similarly, four molecules (F66, F66A, F66B, and F66C), all derivatives of 3-mercapto-1,2,4-triazole, were also added to the OPID library to serve as controls for the RNA binding site of NP. It has been shown that these molecules inhibit the influenza virus by binding to the RNA binding site of NP [215]. Three of the four molecules (F66, F66B, and F66C) served as HACs (HAC1, HAC2, and HAC3, respectively) while F66A served as the LAC (LAC1). Also, two inhibitors of MreB, 
namely A22 and S-benzylisothiourea [250-252] were added to the MF library to serve as the HAC (HAC4) and LAC (LAC2) respectively, for the A22 binding site of MreB. The experimentally determined activities of all control molecules used in this study are shown in Table 2.3.

Table 2.3 Control molecules and their experimentally determined affinities/activities.

\begin{tabular}{ccc}
\hline & \multicolumn{2}{c}{ Control Molecules for the T-Loop Binding Pocket } \\
\hline Control & Target $(\mathrm{H} 1 \mathrm{~N} 1)$ & Activity $\left(\mathrm{IC}_{50}\right) / \mu \mathrm{m}$ \\
\hline HAC & T-loop binding pocket & 2.70 \\
LAC & T-loop binding pocket & 37.50 \\
\hline \multicolumn{2}{c}{ Control Molecules for the RNA Binding Site } \\
\hline Control & Target (H3N2) & Activity (\# of Plaques/Oseltamivir) \\
\hline HAC1 & RNA binding site & $0 \%$ \\
HAC2 & RNA binding site & $4 \%$ \\
HAC3 & RNA binding site & $4 \%$ \\
LAC1 & RNA binding site & $5 \%$ \\
\hline & Control Molecules for the MreB Active Site \\
\hline Control & Target & Activity $($ MIC on E. coli)/ $\mu \mathrm{g} / \mathrm{ml}$ \\
\hline HAC4 & A22 binding site & 3.13 \\
LAC2 & A22 binding site & 100 \\
\hline
\end{tabular}

To specify the search space for GOLD, the T-loop binding pocket and the RNA binding site of NP were each defined as spheres of radii $10 \AA$ centered on the His 272 and Arg174 amino acid residues respectively. The A22 binding site of MreB was defined as a sphere of radius $12 \AA$ centered on Pro112 amino acid residue. In each case, the GOLD GA parameters were set to the virtual screening setting [270]. The prepared molecule libraries 
were docked separately to each distinctive conformation of the appropriate receptor model.

\subsubsection{Ligand selection criteria}

Selection of molecules from the docking results was guided by the following strategy. Ligands were sorted in decreasing order of GOLD scores in docking exercises targeted at MRCs of the T-loop binding pocket of NP, the RNA binding site of NP, and the A22 binding site of MreB as described earlier. Sets of six, five, and four sorted ligand lists for the T-loop binding pocket of NP, RNA binding site of NP, and the A22 binding site of MreB were created, respectively. Regarding the T-loop binding pocket, the common molecules that appeared in the six sorted lists as the top-ranked 50 molecules, 100 molecules, 150 molecules, etc., were selected. The selection was stopped at the level of top 200 for the reason that the LAC emerged at the top 250. For the RNA binding site, the common molecules that appeared in the five sorted lists as top-ranked 10 molecules, 20 molecules, 30 molecules etc., were selected. The selection was terminated at the level of top 50 because LAC1 emerged at the top 60. For the A22 binding site of MreB on the other hand, the common molecules that appeared in the four sorted list as top-ranked 350 molecules, 700 molecules, 1050 molecules, etc., were selected. Here, in line with our experimental plan, we needed at least 10 molecules for X-ray crystallographic studies

with MreB. Therefore, the selection was halted at the level of top 1400 where LAC2 emerged in order to make up the number. Thus we set the cutoff as the common top 200, top 50 and top 1400 molecules for solutions from the T-loop binding pocket, the RNA 
binding site, and the A22 binding site, respectively. The entire method is summarized in Figure 2.1.

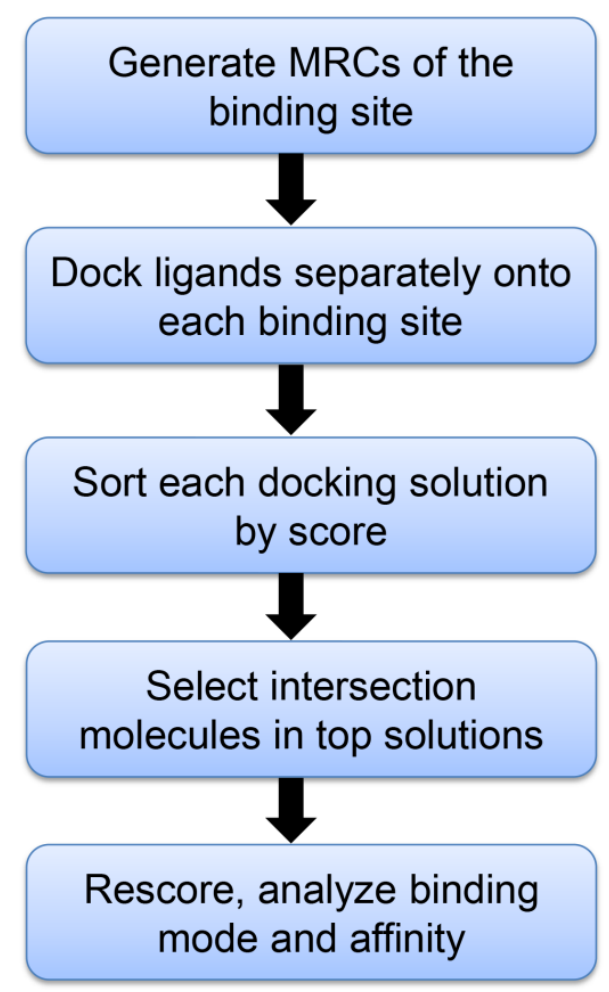

Figure 2.1 Summary of the hit selection criterion. 


\section{Reduction of False Positives in Structure-Based Virtual Screening when Receptor Plasticity is Considered}

\subsection{Introduction}

Although it is currently a daunting task for any docking algorithm to practically explore the entire phase space of a protein receptor, there is enough evidence in support of the need to consider receptor flexibility in SBVS in search of promising drug candidates [182, 183, 186]. It has been reported that rigid receptor docking contributes significantly to inaccurate ligand binding energy estimations and poor binding mode predictions [271]. In spite of the fact that several strategies for incorporating receptor flexibility into molecular docking have been proposed [60, 178-181], docking onto MRCs is considered as the best option that allows one to take advantage of the full flexibility of the receptor [182].

One problem associated with using MRCs in SBVS is that each distinct conformation of the receptor that is introduced into the screening process increases the number of false positives to handle by bringing on board its own set $[172,185,186]$. Thus the selection of true binders or potential drug candidates from the numerous false positives in a molecule library becomes difficult when receptor plasticity is extensively considered in SBVS. The burden and cost of having to deal with false positives are more pronounced where large molecule libraries are screened for potential drugs or lead molecules. In most cases, ligands selected from docking do not have inhibitory effects in experiments [272]; that is to say, most docking results turn out to be false positives. In this study, a ligand selection strategy that could facilitate the selection of true binders from molecule libraries, by 
minimizing the inclusion of false positives into the selection, when receptor flexibility is considered in SBVS was devised.

The binding energy landscape theory [159] was relied on to bring out a hypothesis that a true inhibitor can bind to different conformations of the binding site favorably. To test the hypothesis, sets of distinctive conformations of the T-loop binding pocket and the RNA binding site of NP were generated by MD simulations and used to screen the OP1D library, including control molecules, for true binders using the GOLD docking program $[48,53,129]$. For each binding site, the intersection ligands in the top-ranked molecule lists from all receptor models were selected. The selection strategy successfully distinguished high-affinity and low-affinity control molecules added to the molecule library.

\subsection{Results and Discussion}

Notwithstanding the evidence in support of the need to incorporate receptor flexibility into SBVS [182, 183, 186], there is still no reliable strategy for reducing the false positives it introduces into the predicted candidate inhibitors. In this study, it was sought to devise a ligand selection strategy for reducing the incidence of false positives in SBVS when receptor plasticity is considered. Relying on the binding energy landscape theory [159], a hypothesis that a true binder fits favorably to varying conformations of the binding site was put forth and tested by a search for potential small molecule binders of the T-loop binding pocket and RNA binding site of NP. Receptor plasticity was accounted for by docking and scoring small molecules, including control molecules, in sets of six and five distinctive conformations of the T-loop binding pocket and the RNA binding site 
of NP respectively. The structures used are illustrated in Figure 3.1. To exclude false positives and select true ligands, the common members from all the top-ranked molecule lists obtained by docking the OP1D library, including the control molecules, separately onto all conformations of each binding site were selected. The selection strategy identified all control molecules that were added to the molecule library.

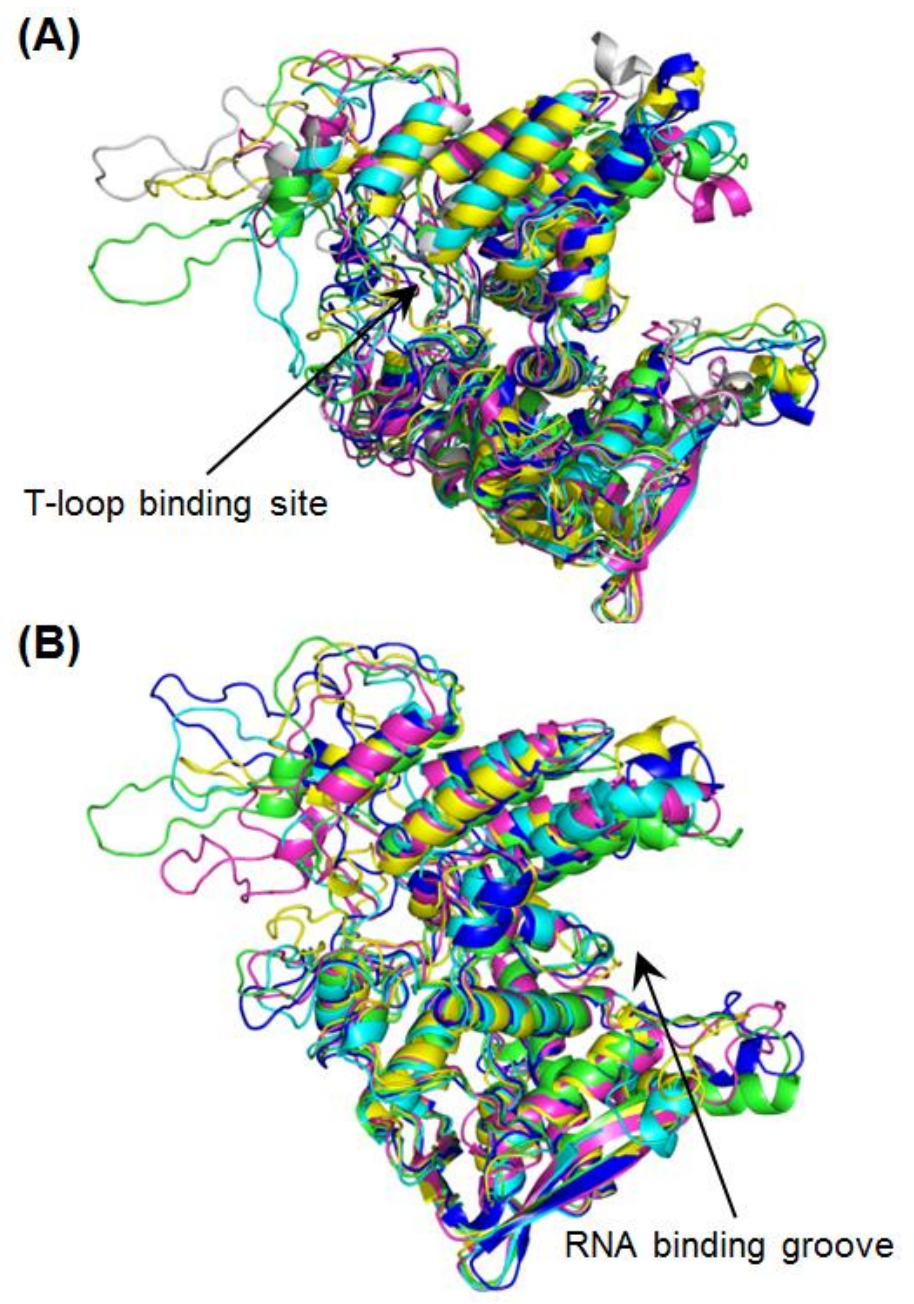

Figure 3.1 Crystal and MD receptor models of NP. (A) Receptor model for the T-loop binding pocket. The crystal structure is colored green. The MD structures 1,2,3,4, and 5 are colored cyan, magenta, blue, yellow, and gray, respectively. (B) Receptor models for the RNA binding site. The crystal structure is colored green. The MD structures 1, 2, 3, and 4 are colored cyan, magenta, blue, and yellow, respectively. 


\subsubsection{Selection by docking to multiple receptor conformations narrows down ligand candidates}

Table 3.1 shows the pattern of selection of molecules by screening the OP1D library against the T-loop binding pocket and RNA binding site of NP. As shown in Table 3.1, for the T-loop binding pocket, only molecule $\mathbf{A}$ belongs to the top 50 molecules of all the six lists. The HAC met the selection criteria at the level of top 100 molecules. The selection of the HAC at top 100 but not top 50 molecules could be attributed to its reported low binding affinity [214] (See Table 2.3). Fourteen molecules were selected from the top 200 lists (Figure 3.2A). It can also be seen in Table 3.1 that no molecule belongs to the top 10 molecules of all the five lists from the RNA binding site. The HACs (HAC1, HAC2, and HAC3) were selected at top 20, 30, and 50, respectively. Seven molecules were selected from the top 50 lists (Figure 3.2B). The selection pattern of the control molecules is consistent with their experimentally determined binding affinities and/or activities $[214,215]$ (Table 2.3). The low-affinity controls (LACs) for both the Tloop binding pocket and RNA binding site were not selected because they were used to set the selection cutoff points. Those molecules that were ranked as top solutions in some lists but ranked lower in other lists could be false positives.

Table 3.1 Pattern of selection of molecules from the Otava Primscreen1 diversity library.

\begin{tabular}{cccccc}
\hline Selection for the T-Loop Binding Pocket & & \multicolumn{2}{c}{ Selection for the RNA Binding Site } \\
\cline { 1 - 2 } \cline { 5 - 5 } Level of Comparison & Molecules Selected & & Level of Comparison & Molecules Selected \\
\hline Top-ranked 50 & A & & Top-ranked 10 & - \\
Top-ranked 100 & HAC and B & & Top-ranked 20 & HAC1 \\
Top-ranked 150 & C-E & & Top-ranked 30 & HAC2 \\
Top-ranked 200 & F-M & & Top-ranked 40 & 1 \\
- & - & & Top-ranked 50 & HAC3, 2-4 \\
Total molecules selected & 14 & & Total molecules selected & 7 \\
\hline
\end{tabular}


(A)

HAC

$\sum_{=N}^{N} \overbrace{}^{n} C^{2}$<smiles>O=C(O)C[C@H](Sc1nc(-c2ccccc2)n[nH]1)c1ccccc1</smiles><smiles></smiles><smiles>COc1ccc(NC(=O)Cn2nnc3sc4c(c3c2=O)CCCC4)c([N+](=O)[O-])c1</smiles>

L<smiles>COc1ccc(-c2nnc(S[C@@H](CC(=O)NCCO)c3cccs3)[nH]2)cc1</smiles>

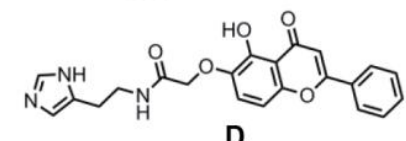

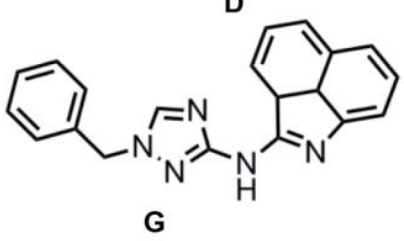

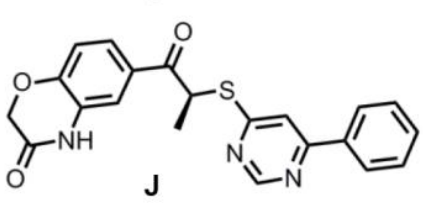<smiles>[Y]C(=CC(Cl)=Cc1ccc([N+](=O)[O-])cc1)SC1NC(=O)NC1N(C)c1ccc(O)cc1</smiles>

$\mathrm{OH}_{\mathrm{OH}}^{C_{\mathrm{H}}}$<smiles>CCNC1=NN=C2C(SC(C)C(=O)c3ccc(Cl)cc3)=NN(NCC)C=NC12</smiles><smiles></smiles><smiles>CCCCCCCC(=O)NCC(=O)Nc1cccc(F)c1</smiles>

(B)<smiles></smiles><smiles></smiles><smiles>CCOc1ccccc1/C=N/NC(=O)CSc1nnc(-c2ccc(Cl)cc2)n1-c1ccccc1</smiles><smiles>C=CCn1c(SCc2ccc(C(=O)NCCO)cc2)nc2ccccc2c1=O</smiles><smiles>C=CCn1c(SCC(=O)Nc2sc(C)c(C)c2C(=O)OCC)nnc1-c1sc(N)nc1C</smiles><smiles>O=C1C(=S)N(CC2CCCO2)C(=O)C1=Cc1c(NCCCn2ccnc2)nc2ccccn2c1=O</smiles><smiles>C=CCn1c(SCC(=O)NCC2CCCO2)nc2scc(-c3ccc(C)o3)c2c1=O</smiles><smiles></smiles>

Figure 3.2 Molecules selected from the Otava PrimScreen1 library. (A) Selected molecules for the T-loop binding pocket. (B) Selected molecules for the RNA binding site. The LACS were not selected but are shown for illustrative purposes. 
The emergence of few molecules in the intersection of top-ranked lists obtained by docking to different conformations of the T-loop binding pocket and the RNA binding site is consistent with reports that each distinctive conformation of the active site that is included into SBVS process brings on board its own set of false positives [185]. However, the emergence of the HACs as intersection molecules in the top-ranked lists give credence to the hypothesis that a true ligand fits favorably to different conformations of the binding site, and to the assertion that the selection criteria leads to the reduction of false positives encountered when receptor flexibility is considered in SBVS.

To further show that the selection strategy identifies true ligands and reduces the false positives brought on board SBVS by distinct receptor conformations, the number of intersection molecules in more than $1,2,3,4$, and 5 conformations of the T-loop pocket and RNA binding site were determined, where applicable (Figure 3.3). As shown in Figure 3.3, the decrease in the number of intersection molecules as the number of conformations increase shows that the technique can greatly narrow down the selection. Since the fewer intersection molecules that emerged with increasing number of conformations do include the HACS (Table 3.1), the observed trend could also mean that the selection criterion is a reasonable way to get rid of the false positives and select true binders. The results also suggest that it is better to include as many conformations of the binding site as possible into the docking process. 
(A)

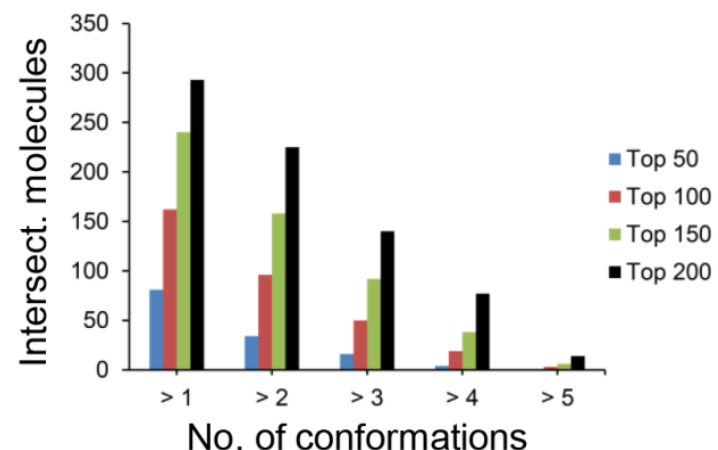

No. of conformations
(B)

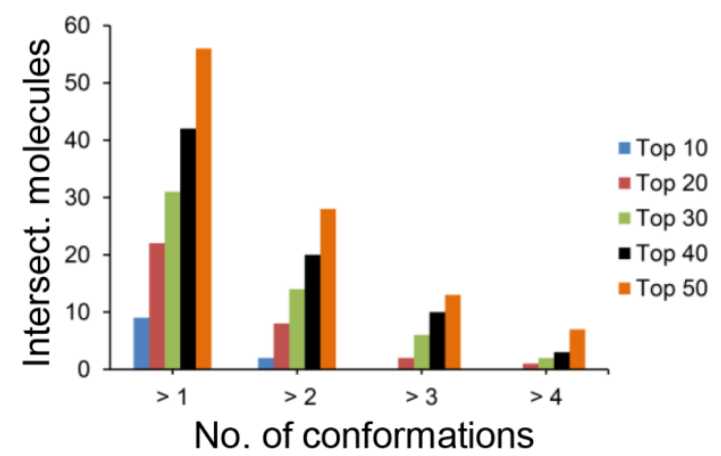

Figure 3.3 Number of intersection molecules in different number of conformations of the T-loop binding pocket and the RNA binding site of NP at different levels of comparison. (A) Number of intersection molecules for the T-loop binding pocket. (B) Number of intersection molecules for the RNA binding site.

To get more accurate binding energies, the selected molecules in the top-ranked 100 molecules docked to the T-loop binding pockets and the entire selection for the RNA binding site were re-docked using the default setting (instead of the high throughput setting) of the GOLD GA parameters [270]. The docking scores are listed in Table 3.2. As shown in Table 3.2, a more potent molecule, molecule A, ranking better than the HAC for binding to all the T-loop binding pockets was found, whereas, for the RNA binding site, no molecule in the library was found to rank higher than the HACs in all conformations. In the following, the focus is on the results of the T-loop binding pockets. 
Table 3.2 Default GOLD scores of selected molecules in all receptor conformations.

\begin{tabular}{ccccccc}
\hline \multicolumn{7}{c}{ Receptor Models for T-Loop Pocket } \\
\hline Molecule & Crystal Str. & Structure 1 & Structure 2 & Structure 3 & Structure 4 & Structure 5 \\
\hline A & 74.17 & 92.21 & 75.70 & 78.95 & 78.85 & 66.77 \\
B & 65.98 & 70.28 & 82.14 & 78.00 & 74.54 & 66.03 \\
HAC & 63.24 & 76.84 & 64.20 & 77.06 & 78.08 & 64.59 \\
\hline \multicolumn{7}{c}{ Receptor Models for RNA Binding Site } \\
\hline Molecule & Crystal Str. & Structure 1 & Structure 2 & Structure 3 & Structure 4 \\
\hline HAC1 & 70.08 & 67.94 & 64.90 & 69.46 & 64.19 & \\
HAC2 & 64.63 & 64.70 & 64.08 & 65.64 & 65.77 & \\
1 & 61.03 & 71.01 & 62.15 & 70.76 & 64.17 & \\
HAC3 & 61.29 & 64.20 & 60.16 & 68.18 & 69.77 & \\
2 & 59.38 & 60.57 & 60.64 & 61.42 & 65.58 & \\
3 & 61.05 & 65.48 & 52.67 & 63.88 & 57.56 & \\
4 & 60.00 & 59.41 & 56.82 & 60.59 & 64.08 & \\
\hline
\end{tabular}

\subsubsection{Molecule A compared to the high-affinity control}

Previous work by Shen et al. [214] suggested that the NP inhibition mechanism by HAC could be emanating from its ability to interact with a Glu339 residue in the T-loop binding pocket. Therefore, to verify that the molecules selected by the criterion described herein are not artifacts, the binding modes and root mean square deviations (RMSDs) of the HAC and molecule $\mathbf{A}$ in the T-loop binding pocket of the crystal structure were compared. Figure 3.4 shows the orientations of the two molecules as predicted by the GOLD docking program $[48,53,129]$. As shown in Figure 3.4A, the HAC forms H-bonds with Gly268, Glu339, and Thr390. The aromatic ring of the molecule is involved in aromatic- $\pi$ interactions with Phe304 and Trp330. This predicted orientation is very close to that of Shen et al. [214]. The predicted binding mode of molecule $\mathbf{A}$ is shown in Figure 3.4B. 
The molecule forms H-bonds with Glu339, Ala387, Thr390 and Ser392. The thiophene group of the molecule forms a ring stacking with Phe458. The ability of molecule A to form more $\mathrm{H}$-bonds could be one of the reasons why it obtains better GOLD scores than the HAC.

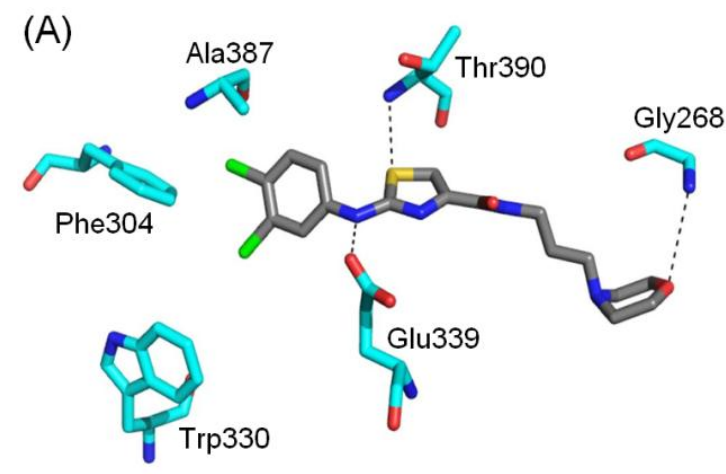

(B)

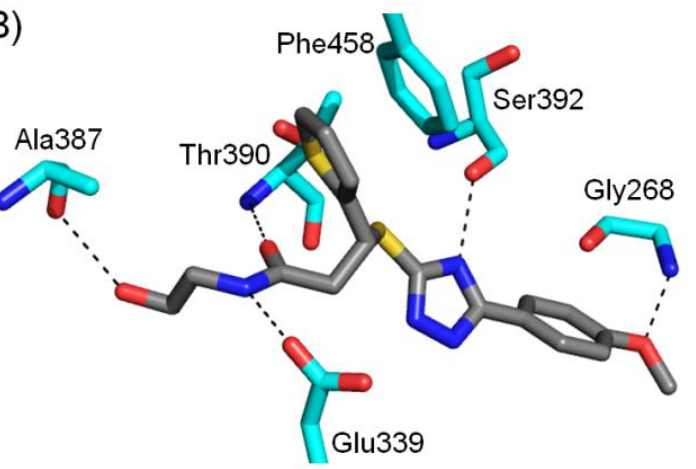

Figure 3.4 Predicted binding modes of the high-affinity control (HAC) and molecule A in the T-loop pocket of the crystal structure of NP. Carbon atoms of the two molecules are colored in dark gray while carbon atoms of NP residues interacting with the molecules are colored in cyan. H-bonds are represented by black dashes. (A) The HAC forms H-bonds with Gly268, Glu339, and Thr390. Its aromatic ring is involved in aromatic- $\pi$ interactions with Phe304 and Trp330; (B) Molecule A forms H-bonds with Glu339, Ala387, Thr390, and Ser392. Its thiophene group forms ring stacking with Phe458.

To test the stability of the predicted binding modes of HAC and molecule A, a $10 \mathrm{~ns}$ simulation of each molecule in complex with the protein in explicit solvent was carried out. Figure 3.5 shows the RMSDs of the two molecules. They both bind stably to the protein with RMSD less than $0.35 \mathrm{~nm}$. Molecule A has even smaller RMSDs. H-bond 
analysis showed that the high-affinity control makes three $\mathrm{H}$-bonds with the protein while molecule A makes four H-bonds.

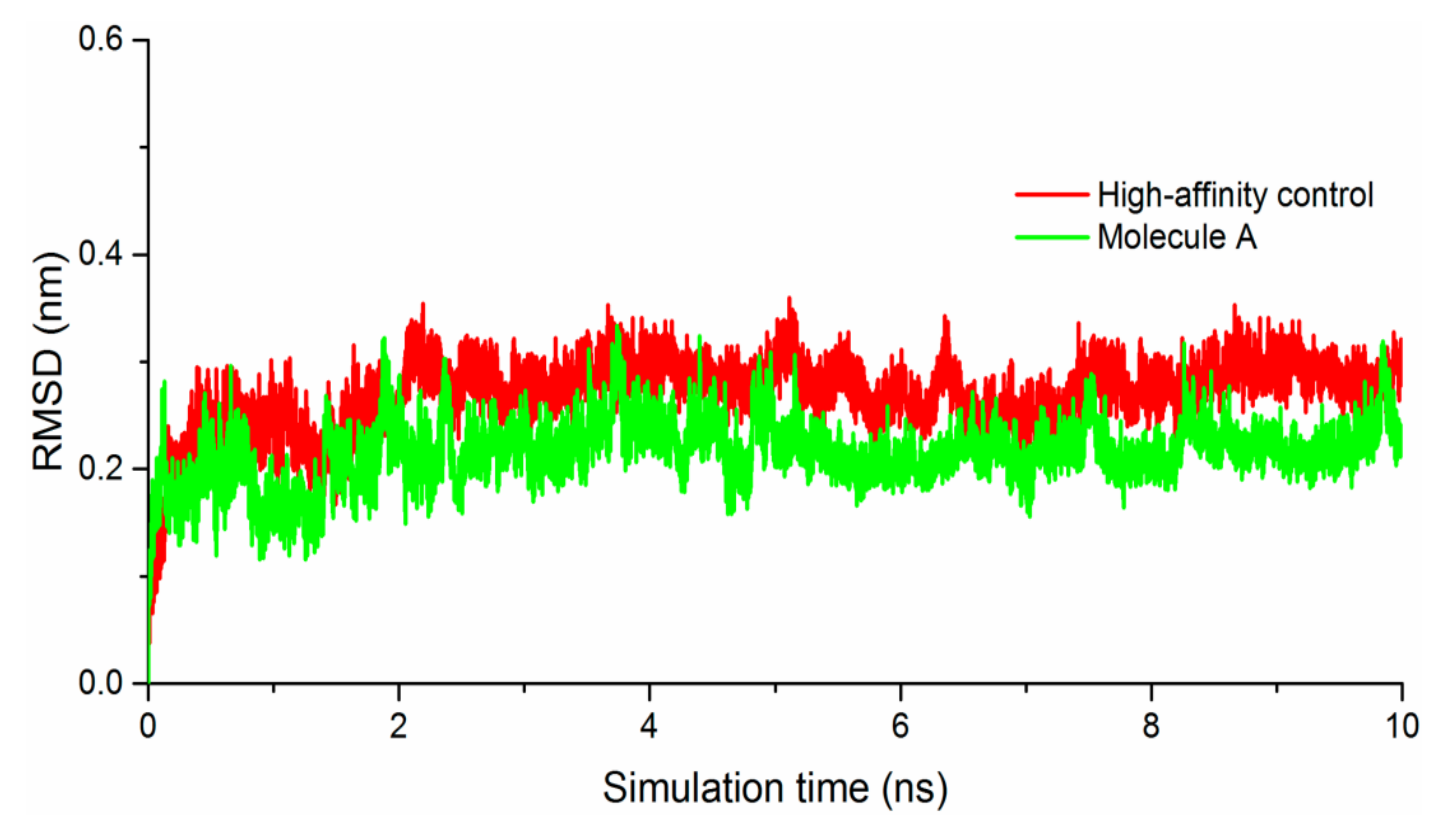

Figure 3.5 Molecular dynamics simulation data. RMSDs of the high-affinity control (HAC) and molecule A in complex with NP T-loop pocket are shown. The RMSDs were calculated on the molecules after least-squares fitting the protein.

\subsubsection{Glu339: A hotspot for molecule A}

By superimposing the receptor-ligand complexes of the MD-generated T-loop-bindingpocket structures onto the crystal structure, the predicted poses of molecule $\mathbf{A}$ are shown in Figure 3.6. In Figure 3.6, one can find that (i) Glu339 is one of the flexible residues in the T-loop binding pocket of NP (ii) molecule A is able to make close contact with Glu339 in all conformations of the T-loop binding pocket through forming H-bonds. 

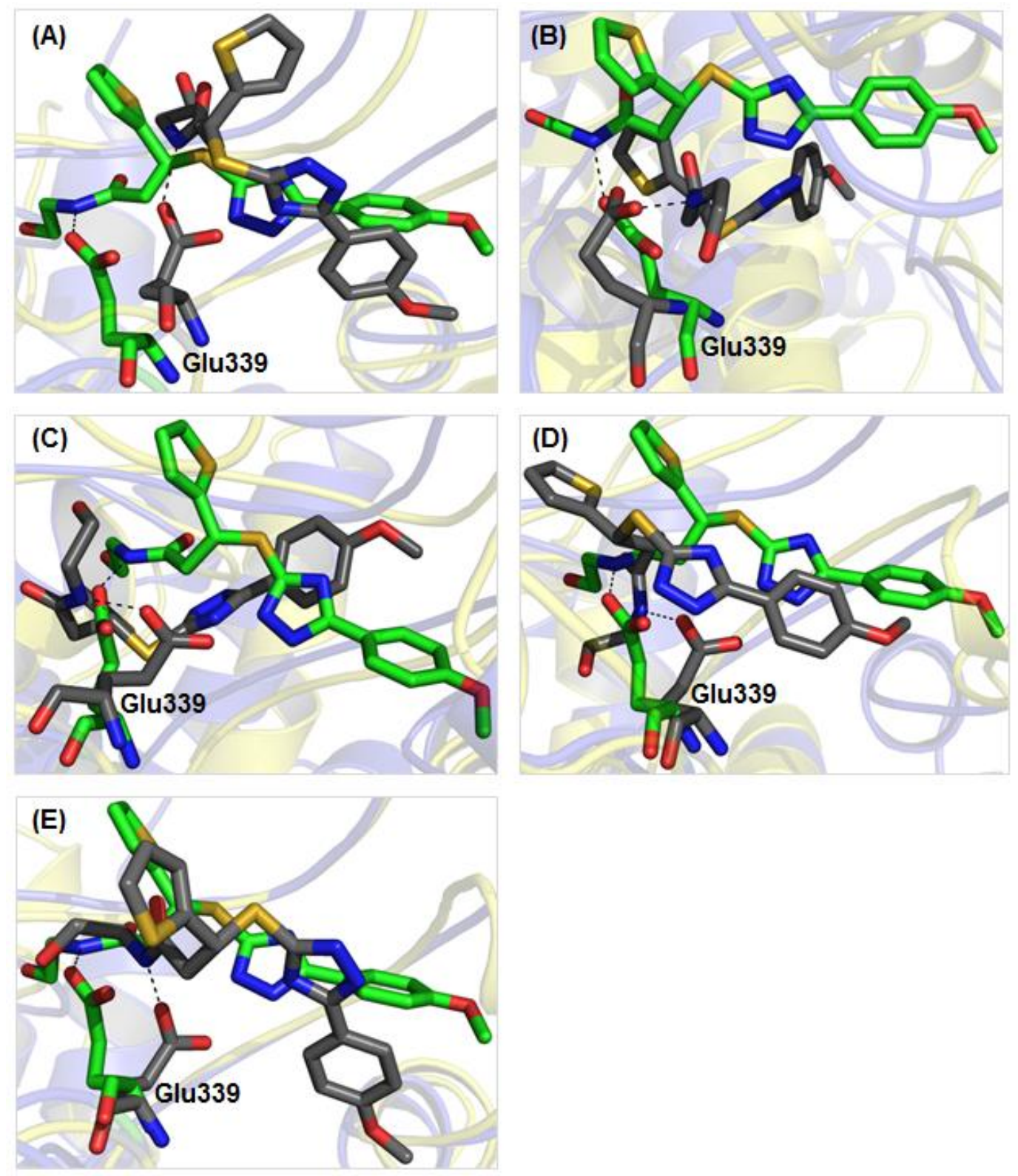

Figure 3.6 Binding modes of molecule $\mathrm{A}$ in the distinctive T-loop binding pocket conformations of NP. The ability of the molecule to interact with Glu339 in all T-loop binding pocket conformations is illustrated. The binding mode of the molecule with the highest score in each conformation of the binding pocket was superimposed on that of the crystal structure. (A-E) Structure 1, 2, 3, 4, and 5 superimposed on the crystal structure. The crystal structure is colored in blue, MD generated structures are in yellow, carbon atoms of Glu339 and molecule $\mathbf{A}$ in the crystal structure are in green. Carbon atoms of Glu339 and molecule $\mathbf{A}$ in MD structures are colored in dark gray. H-bonds between Glu339 and molecule $\mathbf{A}$ are shown in black dashes. 


\subsection{Conclusions}

The main goal of this study was to devise a strategy to reduce false positives and identify true binders when receptor plasticity is incorporated into SBVS. Distinctive multiple conformations of the T-loop binding pocket and RNA binding site of NP were used as receptor models in docking exercises for scoring molecules in the OP1D library, including control molecules. On the basis of the docking scores, sets of six and five separate sortedmolecule lists were created for the T-loop binding pocket and the RNA binding site respectively. The common molecules that sat in the top-ranked solutions of each set of lists were selected.

The ligand selection criterion identified all control molecules added to the library in a manner that is consistent with their experimentally determined binding affinities and/or activities. Molecule A, one of the selected molecules for the T-loop binding pocket, earned better GOLD docking scores than the HAC in all conformations of the pocket. The following MD simulations showed that the binding mode predicted by the GOLD docking program can be maintained stably during 10 ns trajectory. Molecule A could also interact with Glu339 in all conformations of the binding pocket. This behavior of the molecule satisfies a requirement suggested by Shen et al. [214] that molecules that interact with Glu339 could inhibit NP oligomerization.

The hit selection approach described herein provides a means for selecting true binders from a molecule library by reducing the false positives that are usually encountered when receptor plasticity is considered in SBVS. The notion that a true binder fits favorably to different conformations of the binding site roots in the energy landscape theory. Binding, 
like protein folding, has a rugged funnel-like free energy landscape to allow multiple interaction patterns [159]. Structural plasticity of proteins is a natural phenomenon. Theoretically, however, it induces perturbations to the rigid docking process. The described selection criterion selects molecules that are immune to the protein structural variations. In such a way, this method reconstructs the binding energy landscape which is a necessity to describe a true binding process.

\section{NOTE}

The content of this chapter has been published in Molecules. (DOI:10.3390/molecules20035152) 


\section{Validation of Computational Methods}

\subsection{Introduction}

The bacterial actin MreB is important for the determination of non-spherical cell shape $[232,273]$. It functions to support the cytoskeletal network of the bacterial cell much like actin in eukaryotes. MreB has been identified as a possible target for chemotherapeutic treatment of bacterial infections [223, 248, 249]. Small molecules that could bind to MreB and interrupt with its ability to polymerize will disrupt the cytoskeletal network and cause the bacterial cell wall to collapse and eventually lyse [224, 240, 243, 274].

Until recently, it was thought that the known small molecule inhibitors of MreB were all ATP-competitive inhibitors [231, 253, 275]. Thus the ATP binding site of MreB has been the primary target of structure-based drug discovery (SBDD) for the development of antiMreB agents. Limited breakthroughs have been made to this effect [231]. Recently, however, the disclosure by crystal structures [238] that A22 binds to a pocket adjacent to the ATP binding site has been useful in efforts to find potent inhibitors of MreB. Although A22 has some MreB inhibitory effects, it has been shown that it lacks specificity and could have some toxic effects $[276,277]$. This calls for the need to find alternative inhibitors of MreB.

The common practice in drug discovery is to test screening hits for biological activity whereby the active ones are selected for optimization and the inactive ones are rejected. In the face of the need to find new drugs to combat the growing drug resistance [278-281], there is the need to use the few molecule databases that are currently available efficiently 
by broadening the requirements for selecting drug candidates for optimization to include strong binding. Although such strong binders may not have any activity in vivo, which could be caused by poor pharmacological features that are amenable to optimization, they could be used as lead compounds for the development of potent inhibitors. In this study, the hit identification strategy described in the previous chapter was employed to screen the MF library for inhibitors or true binders of MreB using A22 and S-benzylisothiourea as the high-affinity control (HAC4) and the low-affinity control (LAC2) respectively. Eleven hits, representing $0.037 \%$ of the total 30036 molecules making up the MF library were selected for X-ray crystallographic studies involving T. maritima MreB. Three of the hit molecules were detected in the active site of T. maritima MreB. The binding modes of these molecules are close to A22. The results obtained, do validate the computational methods described herein.

\subsection{Results and Discussion}

In the previous chapter, a criterion for identifying true binders when receptor plasticity is considered in SBVS was described [282]. In this chapter, experimental data to support the method are presented. As described earlier, the flexibility of the crystal structure of $C$. crescentus MreB was explored by MD simulations, and three distinct structures of the A22 binding site were generated and then used together with the crystal structure as receptor models in MRC docking exercises using the MF library (including control molecules) and the GOLD program [48, 53, 129].

Figure 4.1, which illustrates the flexibility of MreB, shows the three MD generated structures superimposed on the crystal structure. The crystal structure is colored in green 
while the MD generated structures 1,2, and 3 are colored cyan, magenta, and blue, respectively. The displacement of the protein backbone atoms within the binding site could result in major side chain displacements, even at regions where the backbone displacements are minor and allow the full plasticity of the binding site to be explored. This supports the dynamic nature of proteins and further stresses the need to incorporate a more robust means, such as MD simulations, into molecular docking to account for the flexibility of the receptor; especially when docking programs that claim to explore the flexibility of the receptor only allow slight side chain movements [153-158].

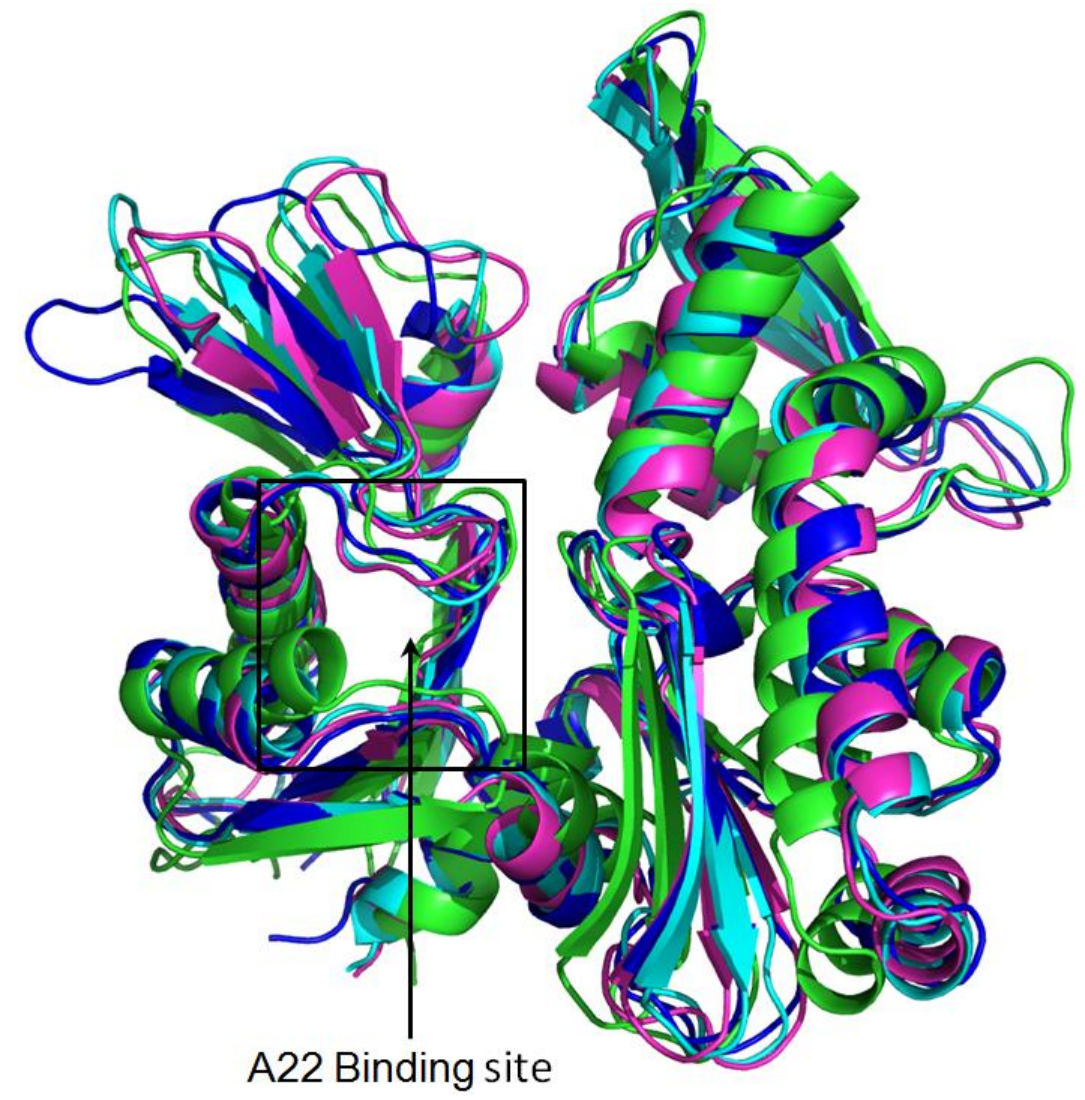

Figure 4.1 Receptor models of MreB. The crystal is colored green, and the three MD structures are colored cyan, magenta, and blue. 
By docking the MF library separately onto the distinct conformations of the A22 binding site of MreB and using the HAC4 and LAC2 to guide the selection of the intersection molecules in the top-ranked solutions as described earlier, 11 molecules were selected for X-ray crystallographic experiments. Two molecules (A1 and B2) and the HAC4 were found in the top 350 molecules of all the four sorted docking lists. Two new molecules (C3 and D4) were selected at the level of top 700 molecules. Only molecule E5 was added to the selected list at the level of top 1050 molecules. And finally, 6 new molecules and the LAC2 were found at the level of top 1400 molecules. One of the objectives of this study was to find molecules that can at least bind to MreB for lead optimization exercises. Therefore, molecules that were identified at the same level as the LAC4 were considered for selection. The selection pattern of the molecules is shown in Table 4.1. The 11 molecules selected for crystallographic studies represent $0.037 \%$ of the total 30036 molecules in the MF library. The chemical structures of the selected molecules and the control molecules are shown in Figure 4.2.

Table 4.1 Pattern of selection of molecules from the Maybridge fragment library.

\begin{tabular}{cc}
\hline Level of comparison & Molecules selected \\
\hline Top-ranked 350 & A1, B2, HAC4 \\
Top-ranked 700 & C3, D4 \\
Top-ranked 1050 & E5 \\
Top-ranked 1400 & F6 - K11, LAC2 \\
\hline
\end{tabular}


<smiles>N=C(N)SCc1ccc(Cl)c(Cl)c1</smiles><smiles>Cc1cc(CSC(=N)N)c(CSC(=N)N)cc1C</smiles><smiles>[R16]c1ccc(OC/C(N)=N\O)cc1</smiles><smiles>N=C(N)SCc1cccc(CSC(=N)N)c1F</smiles><smiles>Cc1ccc(CS/C(N)=N/N)cc1</smiles><smiles>N=C(N)N/N=C/c1ccc(Cl)c(Cl)c1</smiles><smiles>NNC(=O)c1sc2ccccc2c1Cl</smiles>

F6<smiles>N=C(N)NC(=N)Nc1nc(-c2ccccc2)cs1</smiles><smiles>N=C(N)SCc1ccccc1</smiles><smiles>NNc1ncnc2c1sc1ncccc12</smiles><smiles>[B]c1ccc(-c2csnn2)cc1</smiles><smiles>Cc1cc(C)c(NC(=N)NC(=N)NN)c(C)c1</smiles>

Figure 4.2 Chemical structures of molecules selected for crystallographic studies. The control molecules are also shown.

\subsubsection{Selection of intersection molecules in top-ranked docking solutions identifies true binders}

Although C. crescentus MreB was used in the docking process, MreB from T. maritima was used in the X-ray crystallographic experiments. There is a close similarity between MreB from both strains. Figure 4.3 compares the primary, secondary, and tertiary structural properties of MreB from $C$. crescentus and T. maritima. As shown in Figure 4.3A, the overall sequence similarity of MreB from the two strains is $71 \%$, and the residue 
types that define the A22 binding site are well conserved. The secondary and tertiary structural features of $T$. maritima MreB compare very well with those of $C$. crescentus MreB as shown in Figure 4.3B. The catalytic glutamate residue is located at position 140 (Glu140) in the primary sequence of $C$. crescentus MreB, and at position 131 (Glu131) in the primary sequence of $T$. maritima MreB.
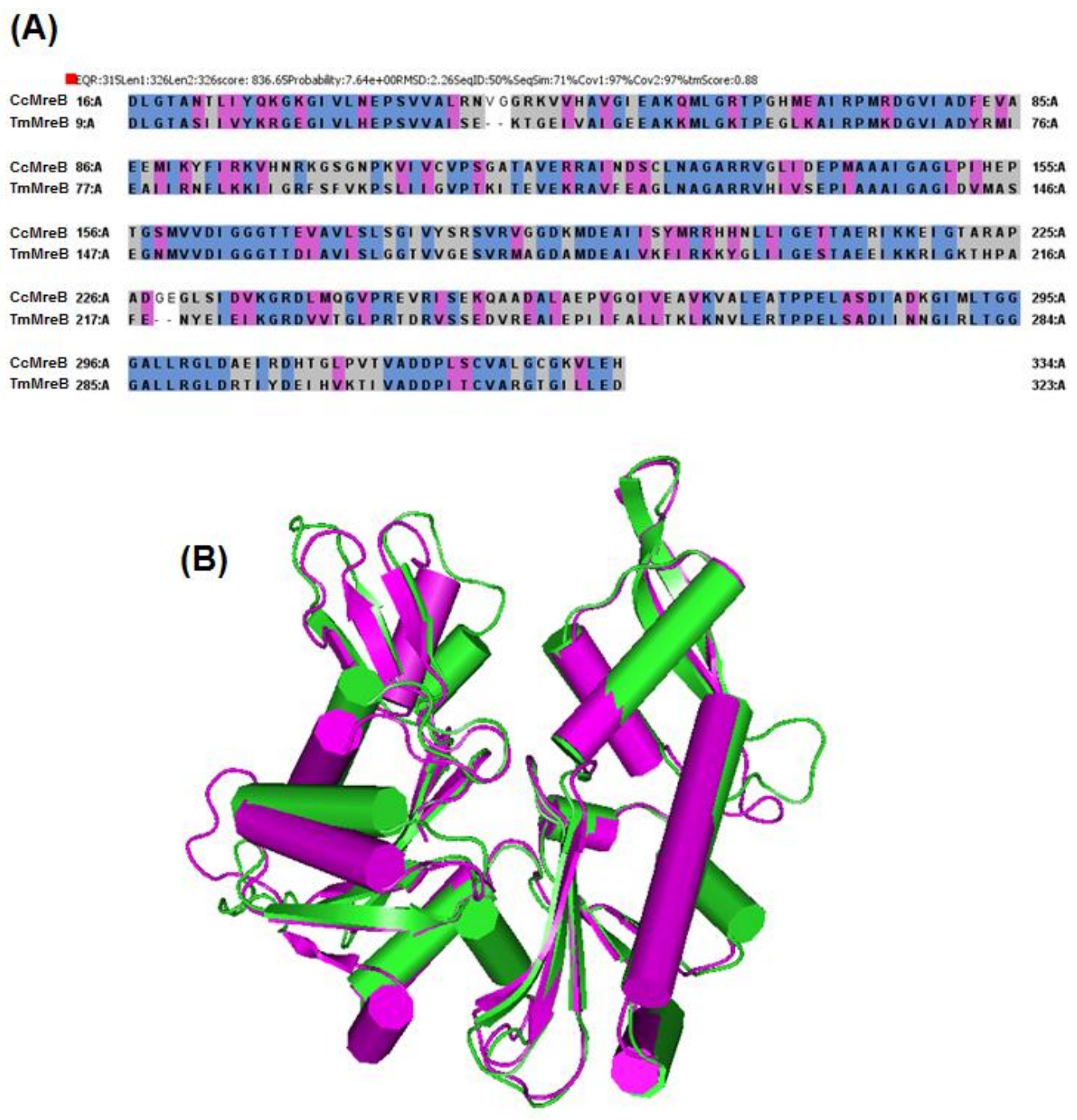

Figure 4.3 Comparison of monomeric C. crescentus MreB (CcMreB) and T. maritima MreB (TmMreB). (A) Comparison of the primary sequence. Blue signifies exact match. (B) Comparison of the secondary and tertiary structural features. Green and magenta colors indicate $C$. crescentus MreB and T. maritima MreB respectively. 
Out of the 11 molecules selected for the crystallographic experiments, the molecules A1, B2, and C3 were found in the active site of T. maritima MreB with clear electron density maps (Figure 4.4). Interestingly, these three molecules were among the best-ranked intersection molecules identified together with HAC4 (Table 4.1). The other molecules were found with LAC2 in the intersection of relatively lower-ranked docking solutions as shown in Table 4.1. LAC2 has been shown to have a poor inhibitory effect on different strains of bacteria $[251,252]$. It is therefore not surprising that the molecules that were poorly ranked with LAC2 were not found in the active site of T. maritima. This outcome is consistent with the claim that the ligand selection strategy described herein identifies true binders, and supports the hypothesis that a true binder can bind favorably to varying conformations of the binding site.

$$
\text { (A) }
$$

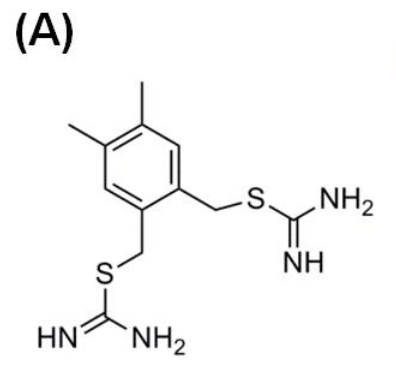

(C)

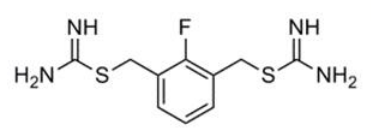

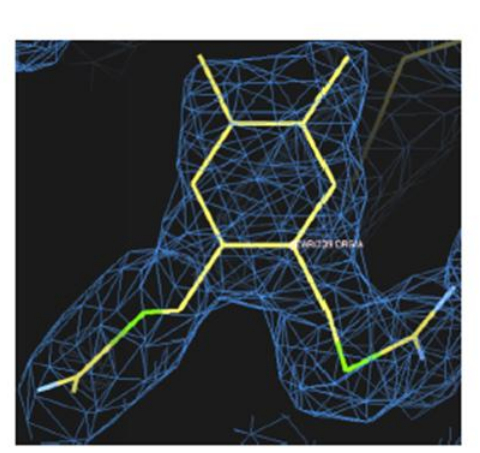

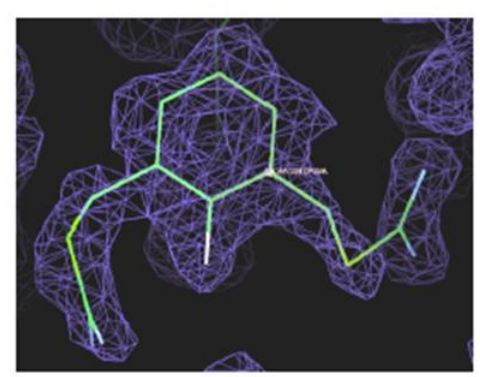

(B)
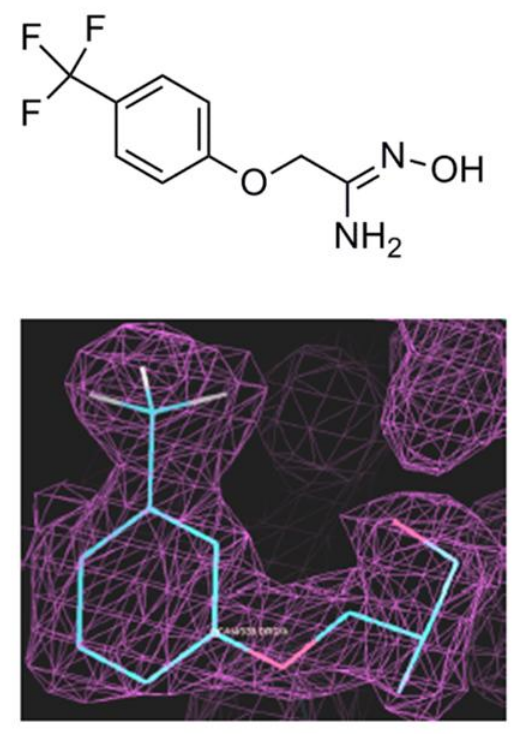

Figure 4.4 Electron density maps of molecules found in the active site of T. maritima MreB. (A-C) Electron density maps of molecules A1, B2, and C3, respectively. 


\subsubsection{Analysis of binding modes of molecules A1, B2, and C3}

Figure 4.5, generated by the ligPlot software [283], illustrates the interactions between HAC4, molecule A1, molecule B2 and molecule C3 with the active site residues of MreB. It can be seen from Figure 4.5B-D that the aromatic rings of all the three molecules fit into the hydrophobic pocket created by Leu17, Gly18, Met74, Ile79, Phe82, Val109, Pro112, Ala115, and Ile123 in a similar way to that of HAC4 (Figure 4.5A). Furthermore, the molecules form H-bonds with the catalytic Glu131 residue as HAC4 does.

(A)

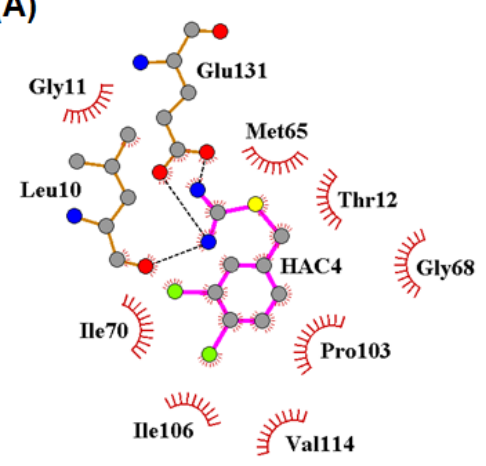

(C)

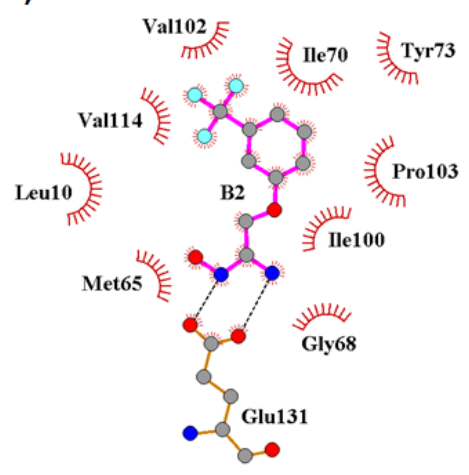

(B)

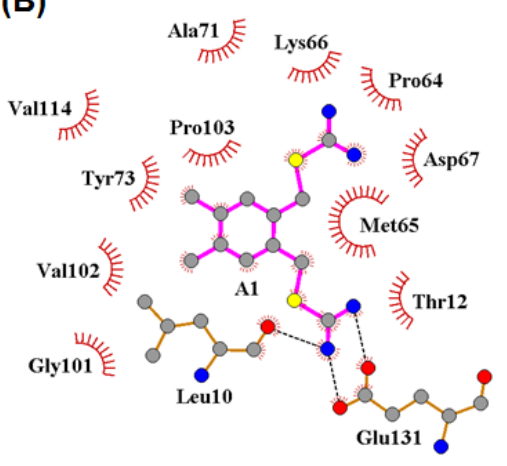

(D)

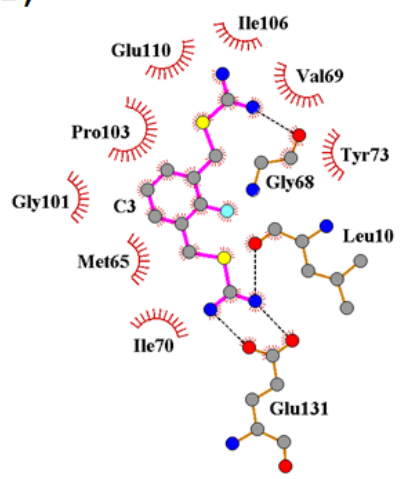

Figure 4.5 Crystal structures of MreB in complex with HAC4, molecules A1, B2, and C3. (A-D) Interactions between the active site residues of MreB and HAC4, molecule A1, molecule B2, and molecule C3. Balls in gray, blue, red, yellow, green, and cyan represent carbon, nitrogen, oxygen, sulfur, chlorine, and fluorine atoms, respectively. Bonds connecting the atoms of ligands are colored in magenta. Bonds connecting the atoms of amino acid residues are colored in orange. The dark red 'eyelashes' represent non-bonded contacts. H-bonds are shown in black dashes. 
HAC4 sits in the active site of MreB together with NT [238]. Crystal structures of HAC4 in complex with MreB [238] and simulation studies (Chapter 5) suggest that HAC4 forms maximum H-bonds with the catalytic Glu131 in the presence of ADP. Since the crystal structures were solved in the presence of ADP, molecule A1-MreB, molecule B2-MreB, and molecule C3-MreB complexes were superimposed on the HAC4-MreB complex to verify how the molecules sit in the active site with ADP. Figure 4.6 shows how the three compounds, as well as HAC4, sit in the active site of T. maritima MreB together with the NT. Clearly, it can be seen that the molecules interact with the catalytic residue as HAC4 does. The safe distance between the molecules and the $\beta$-phosphate of ADP creates favorable conditions that permit maximum H-bond interactions between the molecules and Glu131.

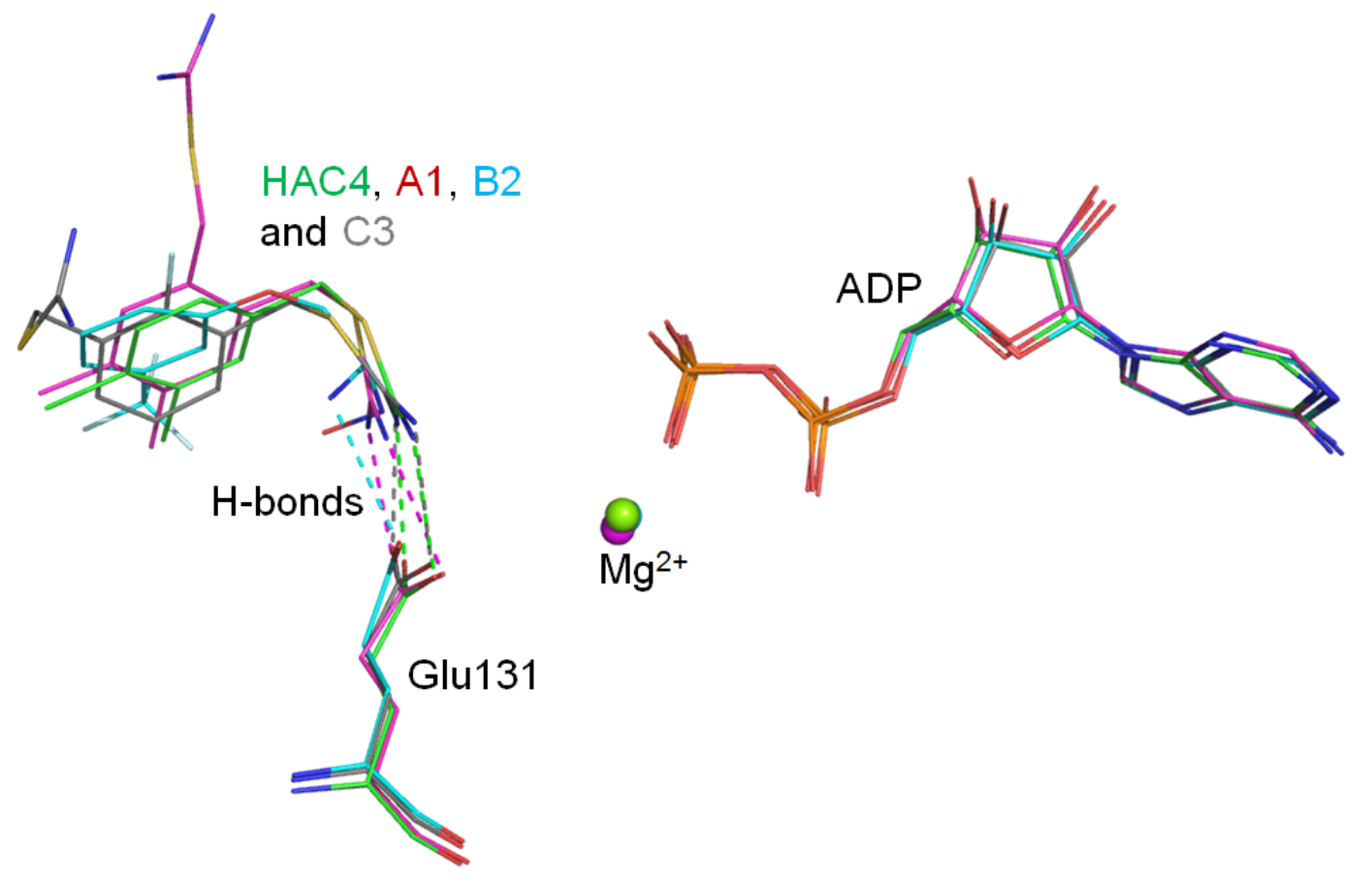

Figure 4.6 Orientations of HAC4, molecule A1, molecule B2, and molecule $\mathrm{C} 3$ in the presence of ADP. H-bonds are shown in dashes. 
The features of a binding site may create a binding environment that favors ligands bearing specific types of chemical structures or pharmacophores. The targeted cavity in the active site of MreB seems to favor molecules that share some structural similarities with A22. It has been shown in SAR studies that changing the parent structure of HAC4 compromises it bioactivity $[251,252]$. The molecules A1, B2, and C3 share some substructural and binding mode similarities with $\mathrm{HAC} 4$, suggesting that the ligand selection criterion described in this study can identify a unique class of small molecule binders of a protein receptor.

\subsubsection{Selection of intersection molecules in multiple receptor docking broadens the conformational and chemical space}

MreB in the molecule A1-bound, molecule B2-bound, and molecule C3-bound states were superimposed on the HAC4-bound state to find out if there are any conformational variations. The result is shown in Figure 4.7. The molecule A1-bound, molecule B2bound, molecule C3-bound, and HAC4-bound states are colored magenta, cyan, gray, and green, respectively. As shown in Figure 4.7, there are no major differences in the structures outside the binding site. In the binding site, however, a loop connecting strand S6 and helix H2 (S6-H2 loop) showed some displacements (Figure 4.7 insert). Interestingly, the flexibility of the S6-H2 loop domain was captured by our simulations and reflected in the MD structures used for the MRC docking (Figure 4.1). Not only does this phenomenon justify our MD simulation protocol but it further stresses the need to dock onto MRCs. 


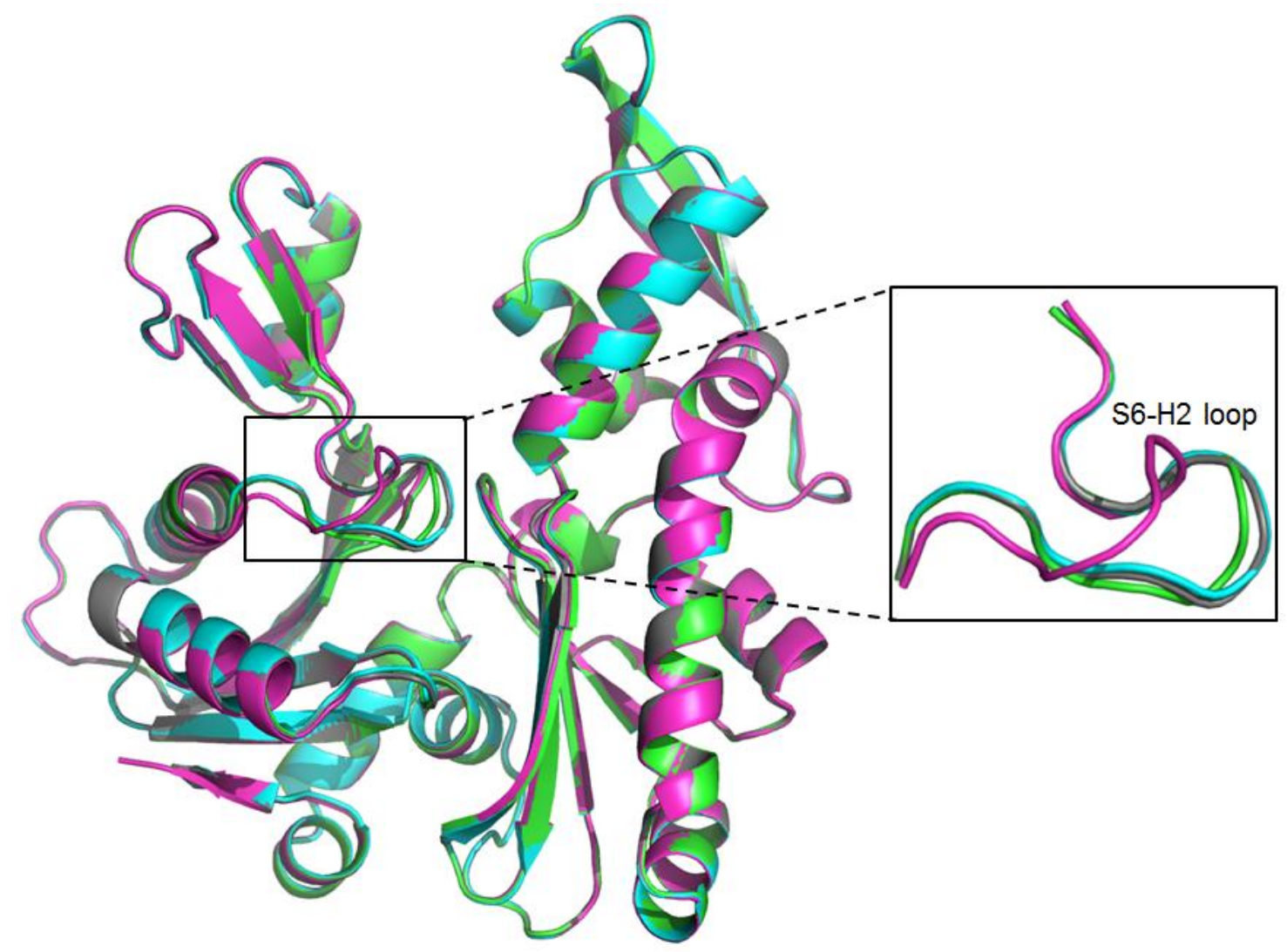

Figure 4.7 Comparison of MreB structures in molecule A1-bound, molecule B2-bound, molecule C3-bound, and HAC4-bound states. Molecule A1-bound, molecule B2-bound, molecule $\mathrm{C} 3$-bound, and HAC4-bound states are colored magenta, cyan, gray, and green, respectively. The insert shows the flexibility of the S6-H2 loop.

A significant displacement of the S6-H2 loop is observed in the molecule A1-bound state where the molecule pushes it outwards in order to bind (Figure 4.7). Indeed, surface diagrams of the molecule A1-bound, molecule B2-bound, molecule C3-bound and HAC4bound states of MreB, as illustrated in Figure 4.8, reveal that the binding site cavity widens in the molecule A1-bound state (Figure 4.8B) as compared to the HAC4-bound (Figure 4.8A), molecule B2-bound (Figure 4.8C), and molecule C3-bound (Figure 4.8D) states. This phenomenon means that docking onto multiple conformations could broaden 
the ligand-accessible space in the receptor to allow promising candidates with varying configurations to bind.
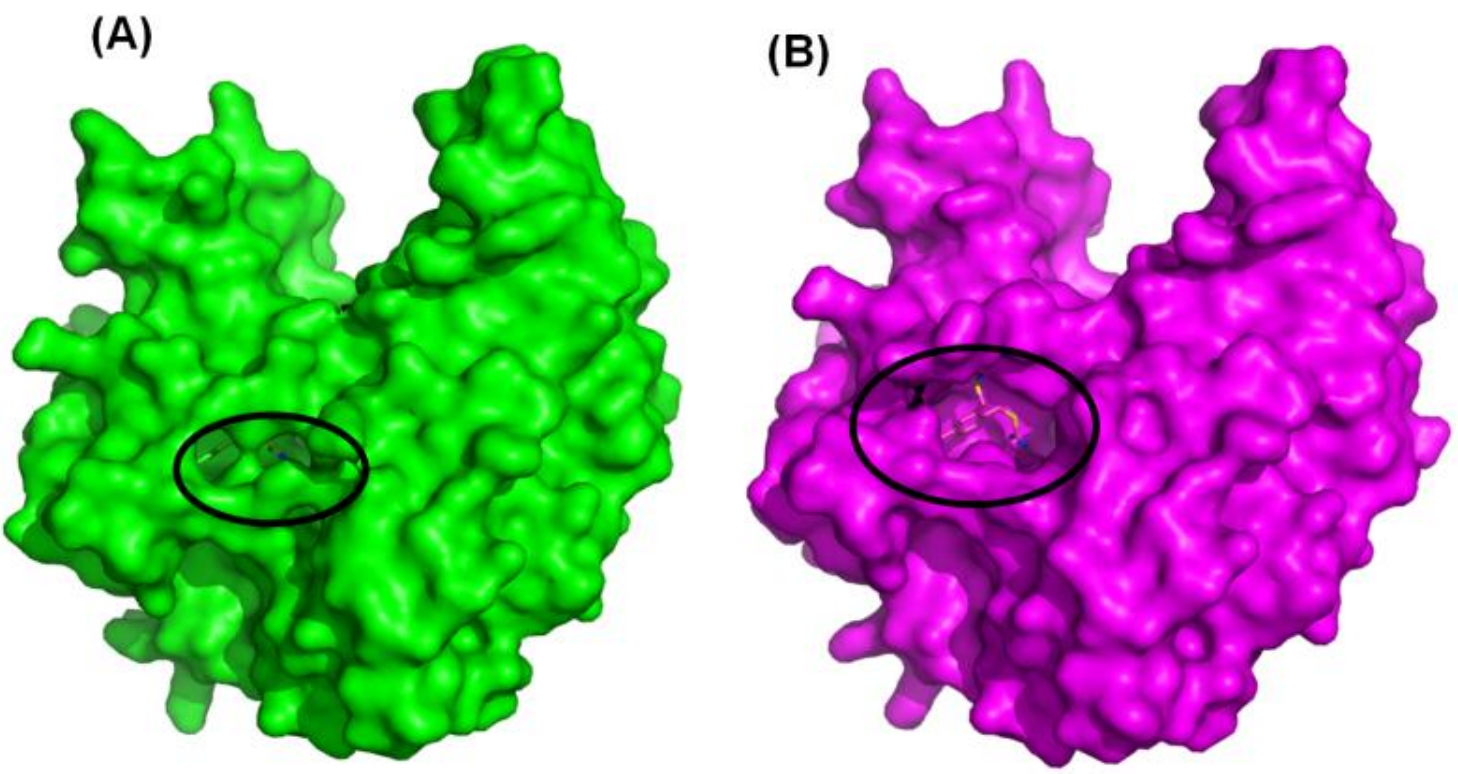

(C)
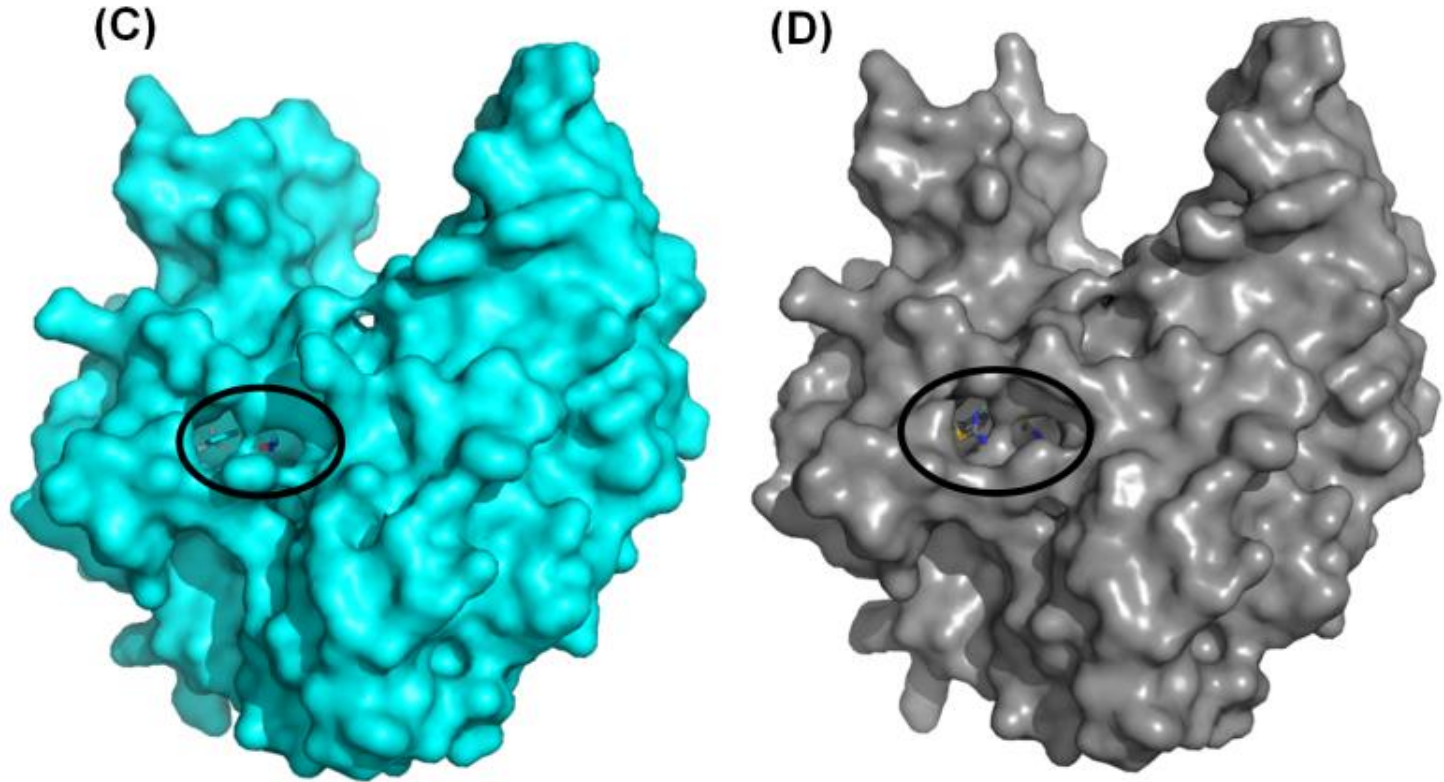

Figure 4.8 Surface representation of MreB in the HAC4-bound, molecule A1-bound, molecule B2-bound and molecule C3-bound states. The black circles indicate the shape of the active site. The HAC4-bound, molecule A1-bound, molecule B2-bound, and molecule C3-bound states are colored green, magenta, cyan, and gray, respectively. 


\subsection{Conclusions}

The main goal of this study was to validate the hits selection criteria described earlier [282] and to find true binders of MreB. The A22 binding site of MreB was targeted with the MF library using the SBVS strategy described in the previous chapter. Eleven out of a total of 30036 molecules were identified as hits and selected for X-ray crystallographic studies. Three molecules $(0.037 \%$ of 30036$)$ were found in the A22 binding site of MreB. The binding modes of the three molecules are close to A22. These molecules could serve as lead compounds for the rational development of MreB inhibitors. The data obtained support and validate the computational methods and the ligand selection criterion described herein. 


\section{Exploring the A22-Bacterial Actin MreB Interaction through Molecular Dynamics Simulations}

\subsection{Introduction}

A22 and its close derivative, MP265 (Figure 5.1A) are small-molecule antibiotics that perturb bacterial cell shape and induce spherical anucleate cells by targeting MreB [243, 250, 284]. It has been demonstrated that A22 binds with micromolar affinity and affects both the time course and the extent of polymerization of MreB into filaments [253]. An initial study suggested that A22 binds to the NT binding site of MreB and thus was hypothesized to be a competitive inhibitor of ATP binding [253]. However, a recent crystal structure of MreB in complex with A22 and AMPPNP, a nonhydrolyzable analog of ATP, provided strong evidence that A22 and ATP simultaneously bind to MreB, where A22 occupies a pocket adjacent to the NT binding site (Figure 5.1B) [238]. The binding affinity of A22 to MreB is high in the presence of NTs [238].

This raises the question: If A22 is not an ATP-competitive inhibitor then what is the inhibition mechanism of A22? On the basis of the resolved structures of ADP-A22-bound and AMPPNP-A22-bound states of MreB, van den Ent et al. [238] suggested that A22 may inhibit MreB in three possible ways: (i) impeding ATP hydrolysis through interactions with the catalytic Glu140 residue and the $\gamma$-phosphate group of ATP, (ii) blocking the channel for the release of hydrolyzed phosphate from the active site, and (iii) perturbing the formation of double protofilaments by means of displacing the main dimerization helix (Ala117-Ala130) at the interprotofilament interface, causing a 
weakening of interprotofilament contacts and a reduction in the stability of double protofilaments.

(A)

(Di)Chlorobenzyl group<smiles>[X]c1ccc(CSC(=N)N)cc1</smiles>

A22: $\mathrm{X}=\mathrm{Cl}, \mathrm{Y}=\mathrm{Cl}$

MP265: $\mathrm{X}=\mathrm{H}, \mathrm{Y}=\mathrm{Cl}$
(B)

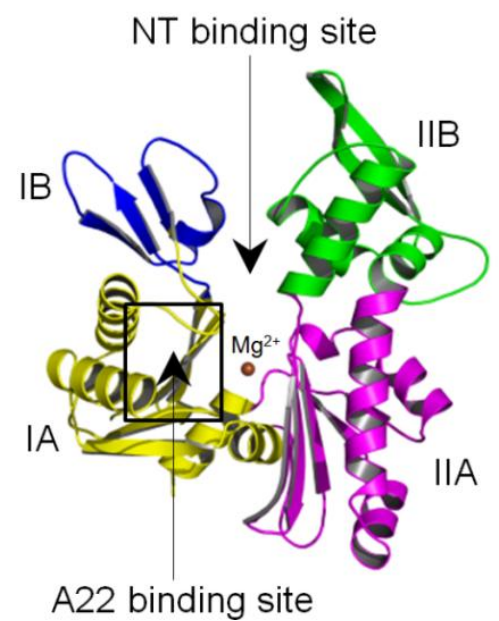

Figure 5.1 Structures of A22, MP265, and MreB. (A) Structures of A22 and MP265 highlighting the (di) chlorobenzyl and isothiourea moieties. (B) Cartoon representation of the monomeric structure of $C$. crescentus MreB. The nucleotide (NT) and A22 binding sites and the four subdomains of the protein are indicated.

The observation that the crystal structure of the ATP-bound state in the presence of A22 has not been solved, despite the ADP-A22- and AMPPNP-A22-bound structures being readily achievable, hints that hydrolysis of ATP by MreB in the presence of A22 is still as fast as when A22 is absent [225]. Thus the hypothesis that A22 inhibits ATP hydrolysis remains an open question. To explore the A22 inhibitory mechanism in detail, it is desirable to reconstruct A22-ATP interaction patterns, which are currently unavailable from experimental structural biology. In this study, MD simulation was applied to explore the dynamics of the active site of MreB in the ATP-A22-bound, AMPPNP-A22-bound, ADP-A22-bound, and NT-free states. The simulations revealed that (i) in the ATP-A22- 
bound and AMPPNP-A22-bound states of MreB, the propensities of A22 for forming $\mathrm{H}$ bonds with the $\gamma$-phosphate of ATP and AMPPNP are higher than its propensities for forming H-bonds with the catalytic Glu140 residue, and (ii) conversely, in the ADP-A22bound state, the propensity of A22 for forming H-bonds with Glu140 is higher than its propensity for forming $\mathrm{H}$-bonds with the $\beta$-phosphate of ADP. Detailed water dynamics analysis hints that in the presence of A22 the conformation of the active site allows water molecules to assume orientations suitable for the hydrolysis of ATP. These observations suggest that in the ATP-A22-bound state of MreB A22 interacts with the $\gamma$-phosphate group of ATP via H-bonds and that this interaction may slow the release of hydrolyzed $\gamma$ phosphate $\left(\mathrm{P}_{\mathrm{i}}\right)$ from the MreB active site.

\subsection{Results and Discussion}

\subsubsection{Validation of simulation protocol and systems}

The primary goal of this study was to apply MD simulations to probe the dynamics of the active site of MreB in different NT-bound states and predict the mechanism of A22 inhibition. Normal MD simulations were carried out as summarized in Table 2.2. To validate the reliability of the simulation protocol and systems, the binding energy of A22 was calculated using the standalone GMXPBSA tool [285]. For each system, 100 frames extracted from the last $50 \mathrm{~ns}$ at intervals of $500 \mathrm{ps}$ were used for the calculations. The quasi-harmonic entropy on A22 was also calculated using the last 50 ns simulations of each system. The entropies were calculated on A22 after fitting the protein structure. The results of the binding energy and entropy calculations are shown in Table 5.1. Each entropy value is an average of calculations from three simulations. The trend of the 
binding energies indicates that the binding of A22 is stronger in the presence of ADP and weaker in the NT-free state, which is consistent with experimental measurements [238].The values obtained for the quasi-harmonic entropies also show that A22 is relatively stable in the presence of NT.

Table 5.1 Table summary of MD simulation systems.

\begin{tabular}{ccccccc}
\hline System & State of MreB & Nucleotide & $\mathbf{A 2 2}$ & $\mathbf{M g}^{\mathbf{2}}$ & Time (ns) & Repeats \\
\hline 1 & ATP-A22-bound & ATP & + & + & 100 & 3 \\
2 & AMPPNP-A22-bound & AMPPNP & + & + & 100 & 3 \\
3 & ADP-A22-bound & ADP & + & + & 100 & 3 \\
4 & Nucleotide(NT)-free & - & + & + & 100 & 3 \\
5 & ATP-bound & ATP & - & + & 100 & 3 \\
6 & ADP-P - -A22-bound & ADP $+\mathrm{P}_{\mathrm{i}}$ & + & + & 100 & 3 \\
7 & ADP-P - -bound & ADP $+\mathrm{P}_{\mathrm{i}}$ & - & + & 100 & 3 \\
\hline
\end{tabular}

Table 5.2 Binding energy and quasi-harmonic entropy of A22 in different nucleotide (NT)-bound states of MreB.

\begin{tabular}{|c|c|c|}
\hline State of MreB & Binding energy(KJ/mol) & Harmonic entropy $(\mathrm{J} / \mathrm{mol} \mathrm{K})$ \\
\hline ATP-A22-bound & $-48.8 \pm 3$ & $208.1 \pm 4$ \\
\hline AMPPNP-A22-bound & $-52.5 \pm 3$ & $209.7 \pm 7$ \\
\hline ADP-A22-bound & $-71.8 \pm 1$ & $206.1 \pm 3$ \\
\hline Nucleotide(NT)-free & $-39.4 \pm 4$ & $250.2 \pm 5$ \\
\hline ADP-P $\mathrm{i}_{\mathrm{i}}$-A22-bound & $-43.4 \pm 4$ & $231.5 \pm 8$ \\
\hline
\end{tabular}

The RMSD of A22 in all repeated runs of the ATP-A22-bound, AMPPNP-A22-bound, ADP-A22-bound, and NT-free states of MreB was also calculated. The RMSDs were calculated on A22 after least-squares fitting the protein. The results are shown in Figure 
5.2. It can be observed that A22 is more stable in the presence of NT than in the NT-free state of MreB. These phenomena support the idea that both A22 and NT bind to the active site of MreB together [238].

(A)

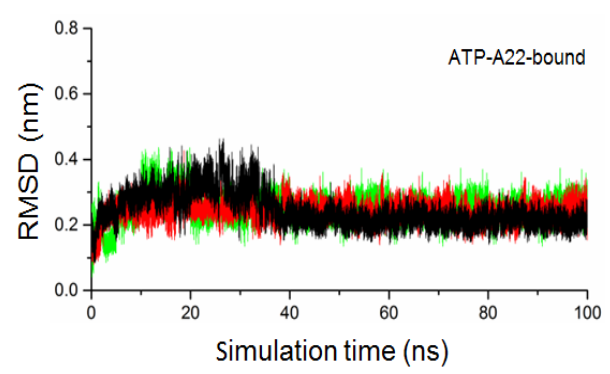

(C)

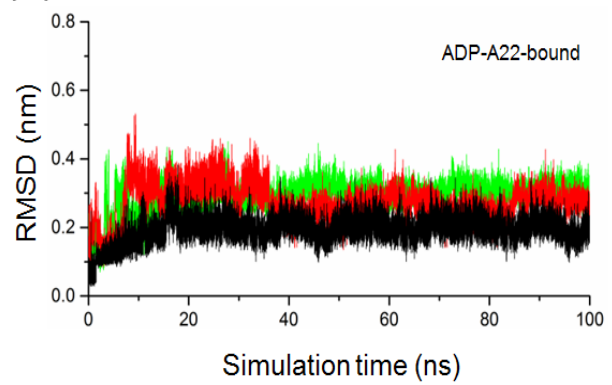

(B)

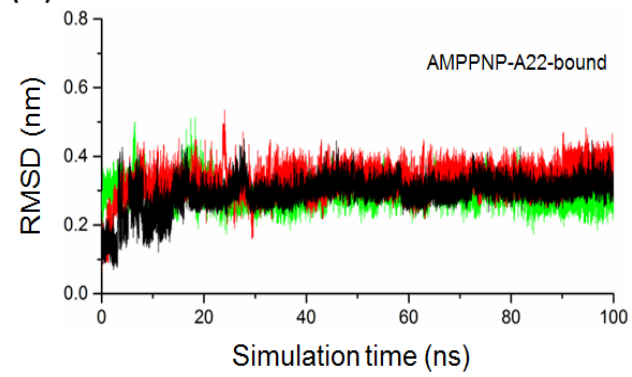

(D)

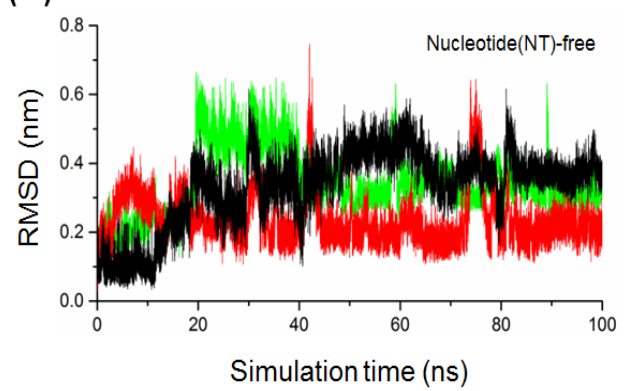

Figure 5.2 RMSDs of A22 in different nucleotide (NT)-bound states of MreB. (A-D) RMSDs of A22 in the ATP-A22-bound, AMPPNP-A22-bound, ADP-A22-bound and NTfree states of MreB, respectively. Black, red, and green colors represent first, second, and third simulations, respectively. The RMSDs were calculated on A22 after least-squares fitting the protein.

\subsubsection{A22 interacts with the $\gamma$-phosphate group of ATP and AMPPNP}

To illustrate the interaction between A22, ATP, and the protein, a representative structure of the ATP-A22-bound state from the simulations is shown in Figure 5.3. To select the representative structure, the last $50 \mathrm{~ns}$ trajectory of simulation 1 of the ATP-A22-bound state was clustered using the gromos method of g_cluster included in the Gromacs 4.5.4 package [265]. With the RMSD cutoff set to $0.12 \mathrm{~nm}$, the protein atoms for structure 
superimposition, and A22 atoms for clustering, the trajectory was grouped into two main clusters. The group-center structure of the largest cluster was picked as the representative structure for the binding mode analysis. The binding mode shown in Figure 5.3 is similar in representative structures from all simulations of the ATP/AMPPNP-A22-bound states. The dichlorobenzyl group of A22 fits well into the hydrophobic pocket created by Leu17, Gly18, Met74, Ile79, Phe82, Val109, Pro112, Ala115, and Ile123 (Figure 5.3). The orientation of the dichlorobenzyl group is very close to that of A22 in the crystal structures. This interaction was well maintained in all simulations carried out.

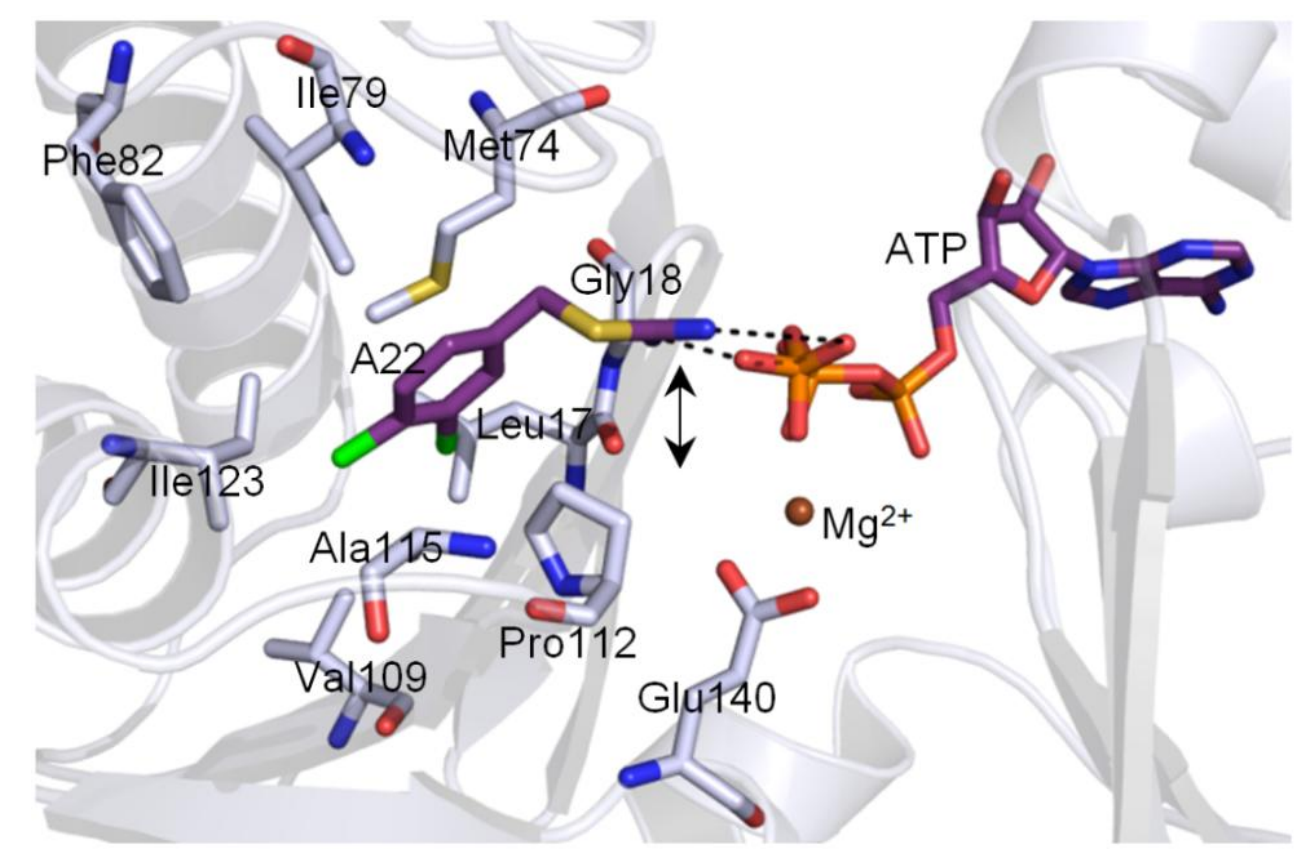

Figure 5.3 Interaction between A22, ATP, and MreB. The H-bonds formed between the isothiourea group of A22 and the $\gamma$-phosphate of ATP are indicated by black dashes. The arrow indicates that the isothiourea group can flip up or down around the sulfur atom.

Interestingly, however, the isothiourea group of A22 was found to undergo conformational changes that enable it to form $\mathrm{H}$-bonds with the $\gamma$-phosphate group of 
ATP. In the X-ray structure of AMPPNP-MP265-bound state (PDB ID: 4CZK), which was modified to the ATP-A22-bound state, the isothiourea group of A22 has a close contact with the side chain of Glu140; that is, the minimum distance between the heavy atoms of the isothiourea group of A22 and the carboxyl group of Glu140 is $2.8 \AA$. This configuration was the initial structure for our simulations. However, as shown in Figure 5.4, the close contact between A22 and Glu140 was not maintained during the simulations. The isothiourea group flips away from Glu140, and meanwhile the H-bonds between A22 and the $\gamma$-phosphate group of ATP are maintained (Figure 5.3). The arrow in Figure 5.3 indicates that the isothiourea group can flip up or down around the sulfur atom. The isothiourea group behaved in a similar manner in the simulations of the AMPPNPA22-bound state.

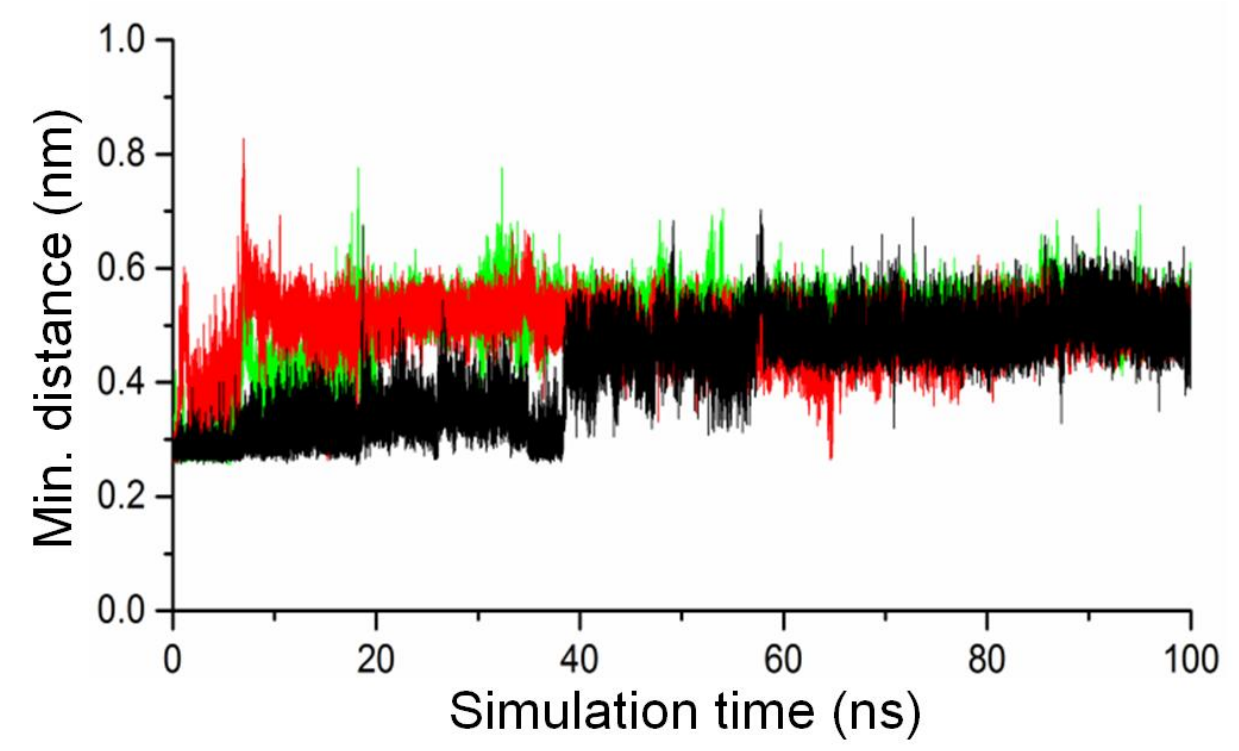

Figure 5.4 Minimum distance between heavy atoms of the isothiourea group of A22 and the carboxyl group of Glu140 in the ATP-A22-bound MreB. Black, red, and green traces represent the minimum distances from the first, second, and third simulations, respectively. 
The flexibility of A22/MP265, more especially the isothiourea group, in the presence ATP/AMPPNP is supported by the experimental B-factor data. Figure 5.5A illustrates the active site of crystal structure of the AMPPNP-MP265-bound state (PDB ID: 4CZK) colored by B-factors. The coloring range has minimum and maximum B-factor values of 0 and 50 respectively. Blue, white, and red colors signify regions of low, average, and high flexibility, respectively. The atoms of the isothiourea group (circled) have high Bfactor values and hence are more flexible and lie in a relatively flexible area of the protein. The sulfur atom has a B-factor as high as $57.2 \AA^{2}$. In contrast, experimental Bfactor data of the active site of the crystal structure of the ADP-A22-bound state (PDB ID: $4 \mathrm{CZG}$ ) suggests that atoms of the isothiourea group of A22 are less flexible and lie in a relatively less flexible area of the protein (Figure 5.5B).
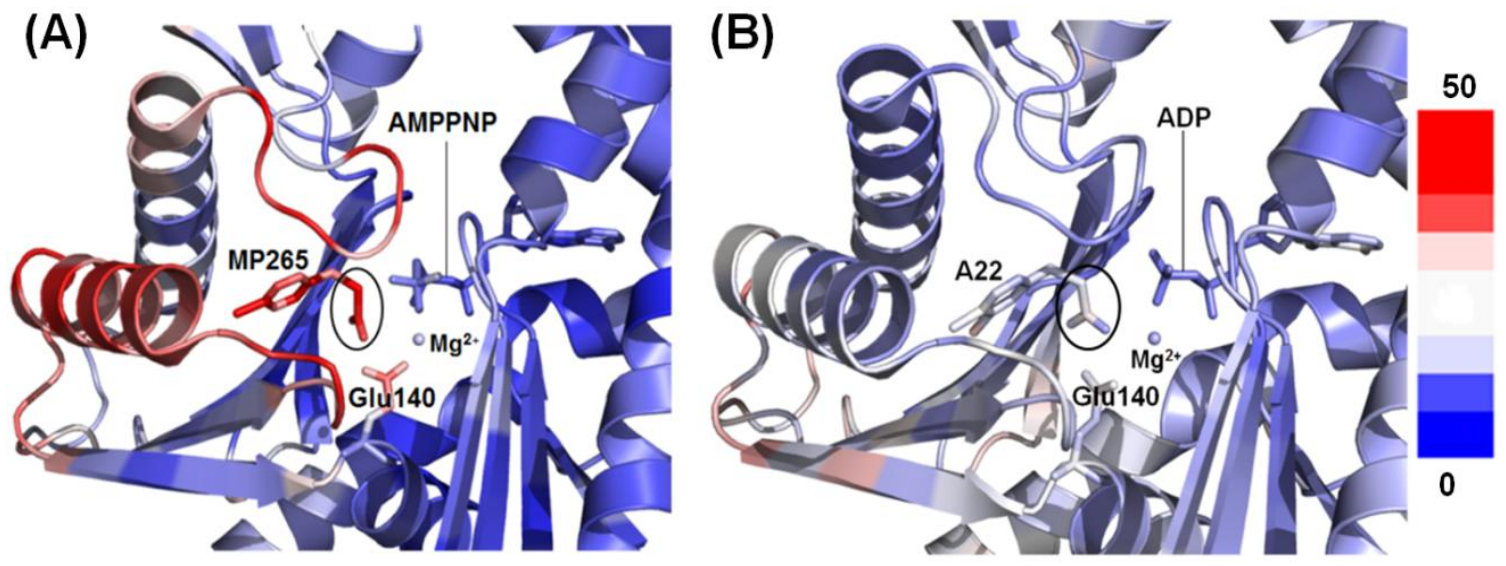

Figure 5.5 Crystal structures of $C$. crescentus MreB colored by b-factor. (A) The AMPPNP-MP265-bound state (PBD ID: 4CZK). (B) The ADP-A22-bound state (PBD ID: 4CZG). The isothiourea groups are circled. 


\subsubsection{Isothiourea group of A22 exhibits interaction preference in different nucleotide (NT)-bound states of MreB}

From the chemical structure of the isothiourea group of A22 (Figure 5.1A), it can be predicted that its interaction with other groups in the active site of MreB would be

primarily H-bond-mediated. Therefore, the propensities of A22 for forming H-bonds with the catalytic Glu140 residue and the NT (where applicable) in the different NT-bound states of MreB were estimated. The time-dependent H-bond probabilities for each state of MreB are shown in Figures 5.6 and 5.7. The overall averaged probability for each state was obtained from the last $50 \mathrm{~ns}$ trajectories. The averaged probabilities are presented in Figure 5.8. Each point is an average of probabilities from three simulations. Interestingly, it can be observed from Figure 5.8 that A22 forms H-bonds with the catalytic Glu140 residue only in the ADP-A22-bound state. While in the ATP/AMPPNP-A22-bound states, A22 forms stable H-bonds with the $\gamma$-phosphate group of ATP/AMPPNP and does not interact with Glu140. 
(A)

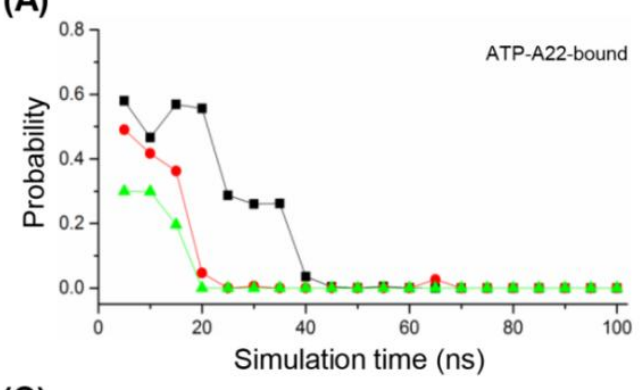

(C)

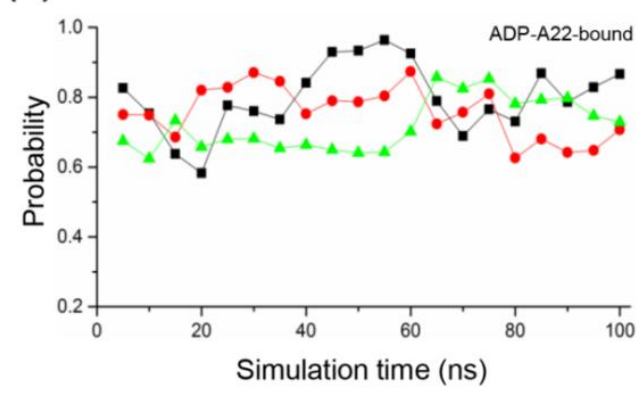

(B)

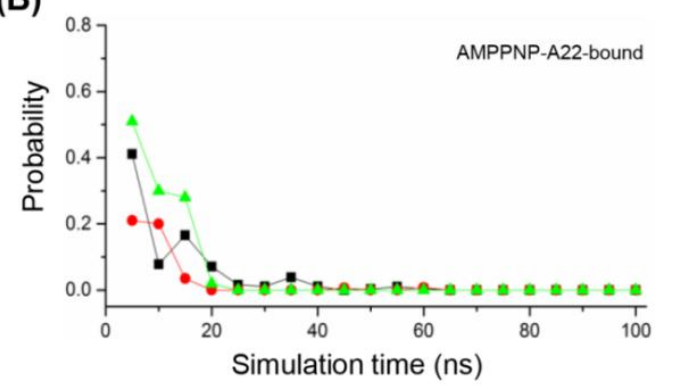

(D)

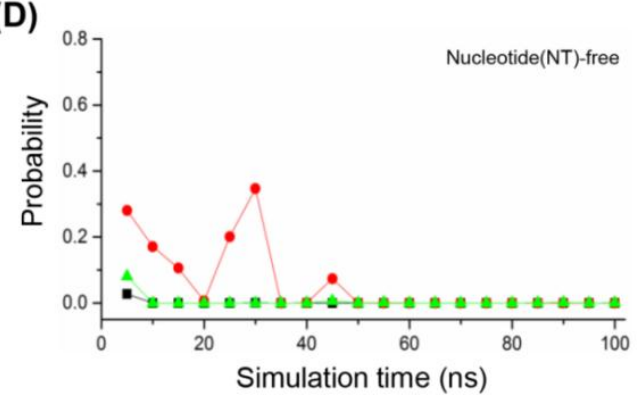

Figure 5.6 Data for H-bond formation probabilities between A22 and Glu140. (A-D) Probabilities of H-bond formation between A22 and Glu140 in the ATP-A22-bound, AMPPNP-A22-bound, ADP-A22-bound, and nucleotide (NT)-free states of MreB. Black, red, and green plots represent probabilities from the first, second, and third simulations, respectively. The criterion for selecting H-bonds was H-bond length not exceeding $3.5 \AA$. The probabilities were calculated after every $5 \mathrm{~ns}$. 
(A)

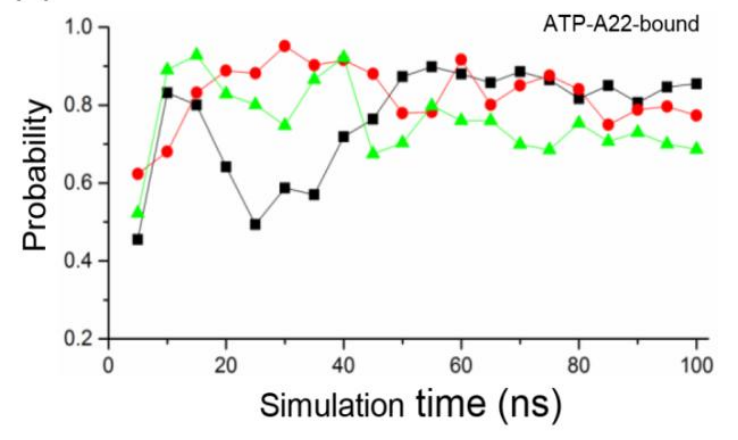

(B)

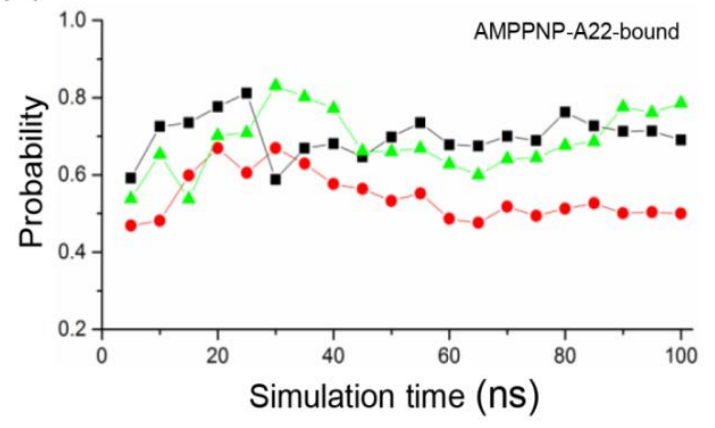

(C)

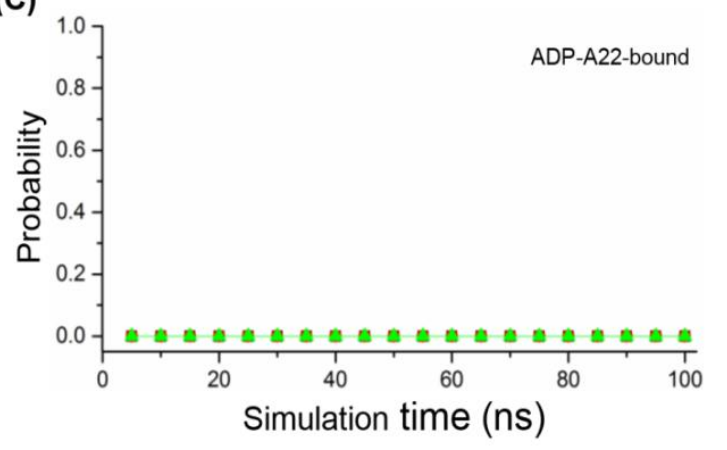

Figure 5.7 Data for H-bond formation probabilities between A22 and nucleotides. (A-C) Probabilities of H-bond formation between A22 and phosphate in the ATP-A22-bound, AMPPNP-A22-bound, and ADP-A22-bound states of MreB. Black, red, and green plots represent probabilities from the first, second, and third simulations, respectively. The criterion for selecting H-bonds was H-bond length not exceeding $3.5 \AA$ A. The probabilities were calculated after every $5 \mathrm{~ns}$. 


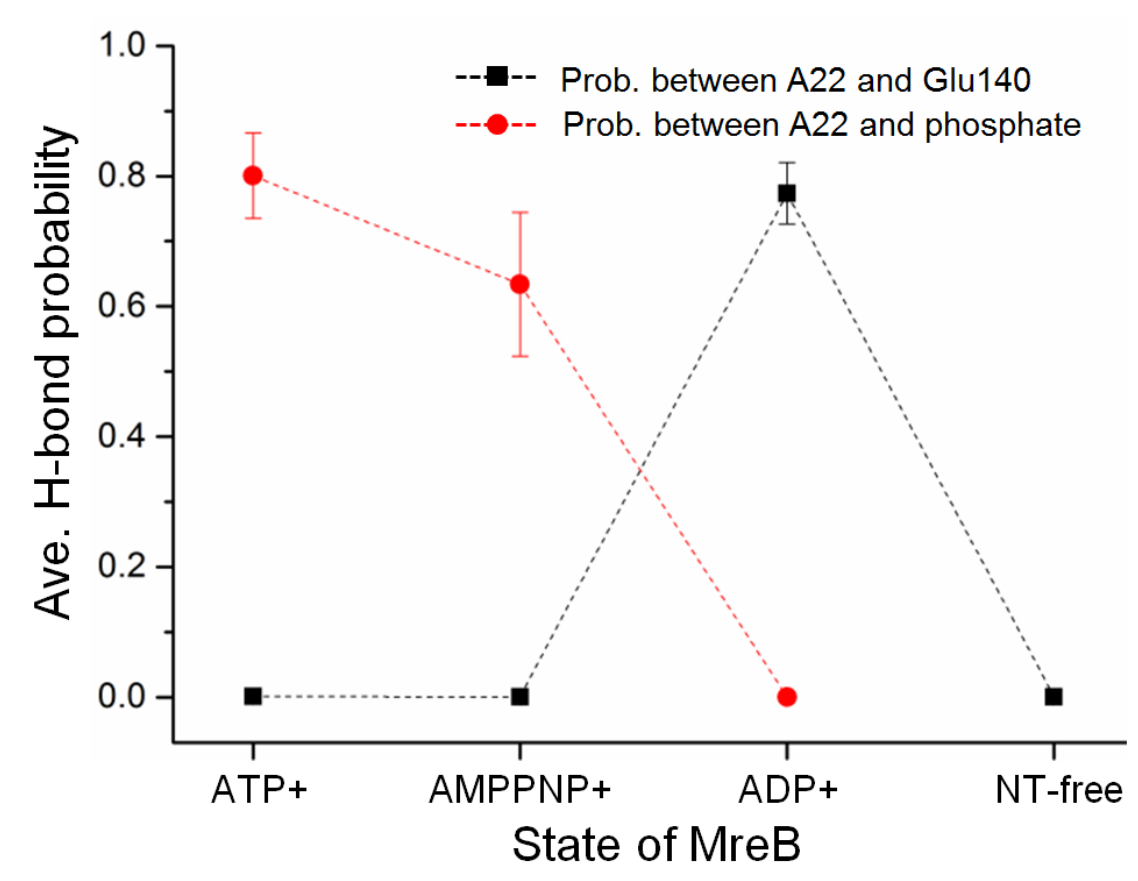

Figure 5.8 Averaged probabilities of $\mathrm{H}$-bond formation by A22. The black square symbols represent averaged probabilities of H-bond formation between A22 and Glu140, and the red circle symbols represent averaged probabilities of H-bond formation between A22 and phosphate groups in different nucleotide (NT)-bound states of MreB. Each data point is an average of probabilities from three simulations.

The catalytic Glu140 plays the following roles during ATP hydrolysis: (i) It helps to localize the catalytic water through hydrogen bonding and (ii) it acts as a base to facilitate the transfer of a proton from the catalytic water to generate a nucleophile, which mounts a nucleophilic attack on the $\gamma$-phosphorous atom of ATP [286, 287]. Therefore, a small molecule that forms stable H-bonds with Glu140 could disrupt the localization of lytic water and the generation of the attacking nucleophile. Thus our finding that A22 barely forms H-bonds with Glu140 in the ATP-A22-bound state hints that the presence of A22 may not affect the hydrolysis of ATP and may explain why the crystal structure of the ATP-A22-bound state of MreB is not tractable. 
The poor binding energy (Table 5.1), high harmonic entropy (Table 5.1), and the characteristic high RMSD fluctuations (Figure 5.2D) recorded by A22 in the NT-free state suggest that A22, specifically the flexible isothiourea moiety, is mobile in the absence of NT. It appears that the absence of NT lessens the steric impediments that restrict conformational changes of the isothiourea group, and this could be the reason why A22 does not bind Glu140 in the NT-free state. This phenomenon suggests that the presence of NT enhances A22 binding and stability.

\subsubsection{Water dynamics and ATP hydrolysis at the active site}

The dynamics of water molecules in the NT-A22 binding site was analyzed based on the final 50 ns trajectory of the ATP-A22-bound state of MreB. The solvent-accessible space near the $\gamma$-phosphate of ATP and the catalytic Glu140 residue is termed as the catalytic zone, being crucial for ATP hydrolysis, which is defined as the intersection of two spheres with the radii of $3.9 \AA$ and $3 \AA$ around the phosphorus atom $\left(\mathrm{P}_{\gamma}\right)$ of the $\gamma$-phosphate of ATP and the carbonyl oxygen atom (OE1) of Glu140, respectively [288-290]. The water molecules that entered the catalytic zone were counted. During the final 50 ns trajectory, a total of 165 water molecules were found to enter this zone. Figure 5.9A illustrates the time spent by the water molecules in the catalytic zone. While the majority of water molecules spent just a few picoseconds, water molecules, W12, W76, W121, and W147, stayed much longer, ranging from 1.7 to $2.3 \mathrm{~ns}$ in the catalytic region. Thus the long lifetimes of W12, W76, W121 and W147 at the catalytic site could be indicative of the localization catalytic water for ATP hydrolysis. Water molecule W3 constantly appeared in the

catalytic zone because it is one of the $\mathrm{Mg}^{2+}$-coordinated water molecules. In a similar 
analysis of the dynamics of water in the active site of the ATP-bound state, which served as the control system, a total of 196 water molecules were found to enter the catalytic zone. As shown in Figure 5.9B, the overall water dynamics in the ATP-bound state look similar to that of the ATP-A22-bound state. Similar results were obtained for all simulations of the ATP-A22- and ATP-bound states.

\section{(A)}

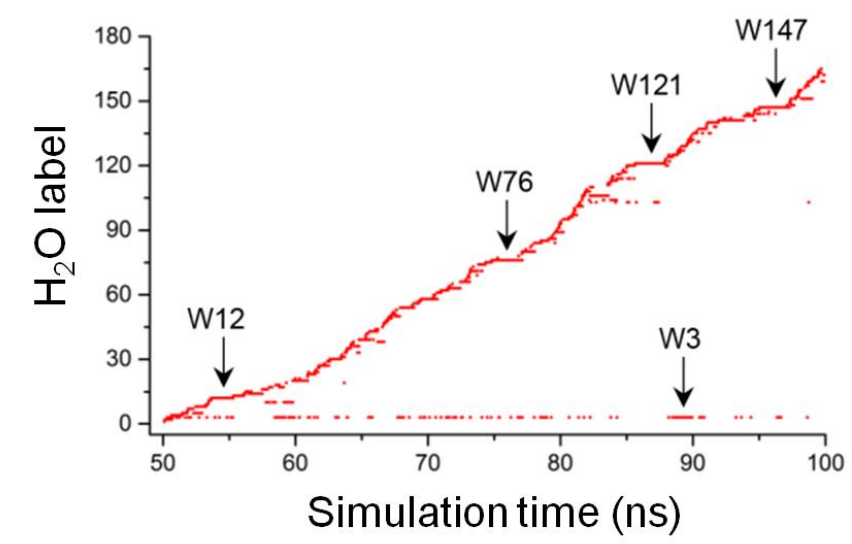

(B)

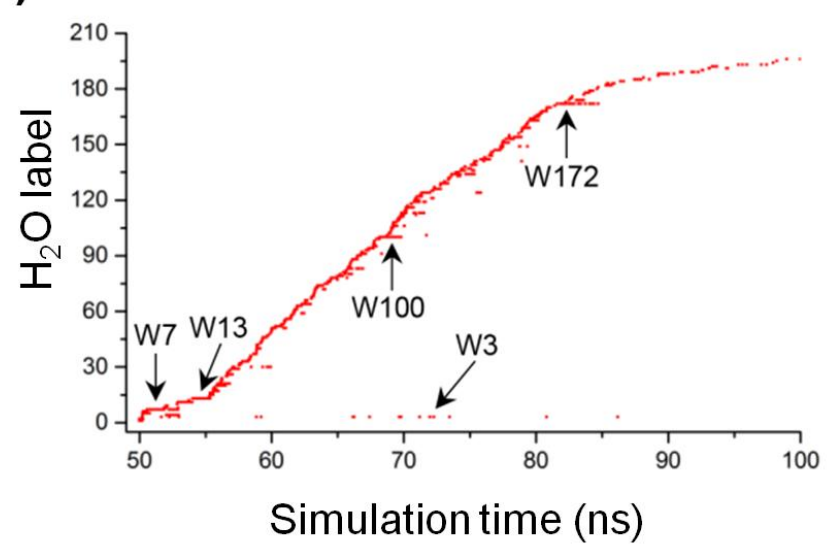

Figure 5.9 Time spent by water molecules in the catalytic zone of MreB. (A) Time spent by water molecules in the catalytic zone of the ATP-A22-bound state. (B) Time spent by water molecules in the catalytic zone of the ATP-bound state. The catalytic zone is defined as the intersection of two spheres with the radii of $3.9 \AA$ and $3 \AA$ around the atoms P $\gamma$ of ATP and OE1 of Glu140, respectively. The y-axis (H20 label) represents the numbering of the water molecules found in the catalytic zone. 
Figure 5.10 shows how the long-staying water molecules are positioned in the active site. The distance " $\mathrm{d}$ " from the oxygen atom $\left(\mathrm{O}_{\mathrm{w}}\right)$ of the water molecule to $\mathrm{P}_{\gamma}$ and the angle " $\alpha$ " between the axis defined by $\mathrm{O}_{\mathrm{w}}$ and $\mathrm{P}_{\gamma}$ atoms and the axis defined by $\mathrm{P}_{\gamma}$ and the oxygen 3 atom $\left(\mathrm{O}_{\beta}\right)$ of the beta phosphate of ATP meet the requirements for ATP hydrolysis [288290]. This orientation is similar for all long-staying water molecules in the ATP-A22- and ATP-bound states. The finding of long-lived water molecules at the active site indicates that in the presence of A22 the conformation of the active site provides a docking platform for a water molecule to initiate the hydrolysis process.

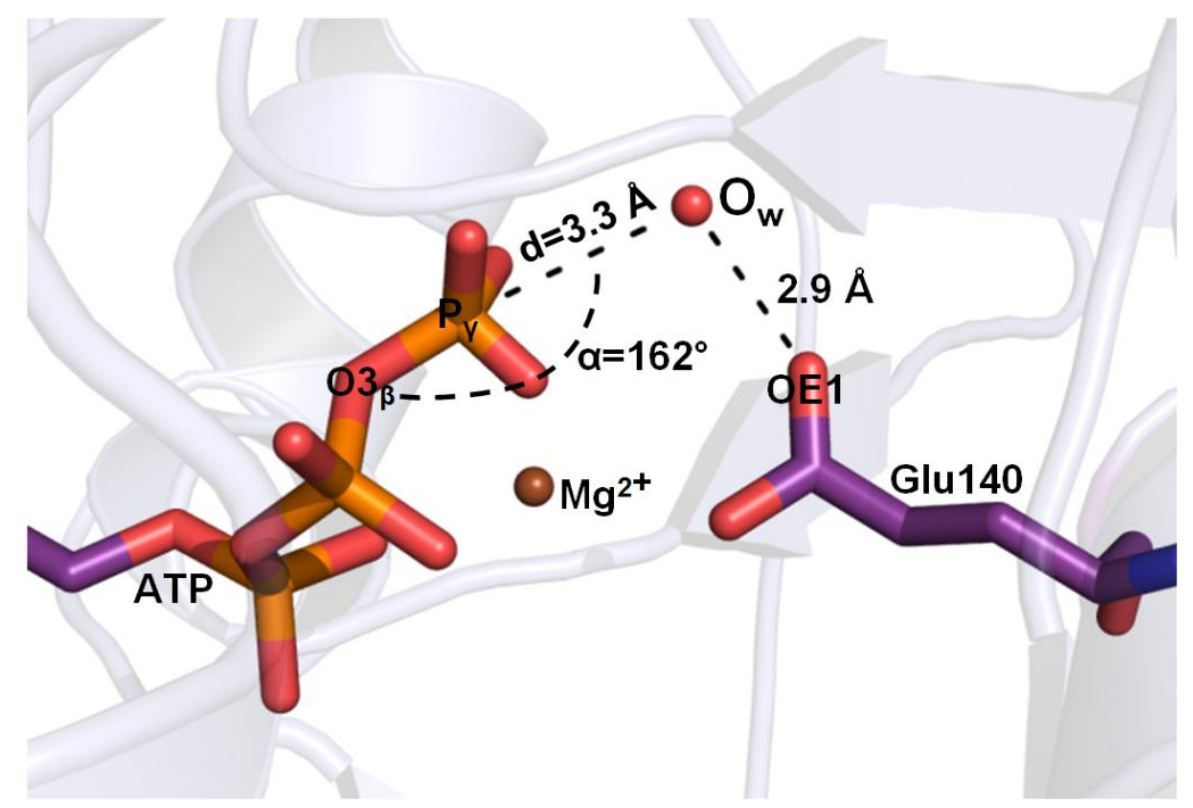

Figure 5.10 Configuration of long-staying water molecules at the active site. " $d$ " is the distance between the atoms $\mathrm{O}_{\mathrm{w}}$ to $\mathrm{P}_{\gamma}$. " $\alpha$ " is the angle defined by atoms $\mathrm{O}_{\mathrm{w}}, \mathrm{P}_{\gamma}$, and $\mathrm{O} 3_{\beta}$.

\subsubsection{Stable hydrogen bonds are formed between $A 22$ and $P_{i}$}

To find out whether the interaction between $\mathrm{A} 22$ and $\mathrm{P}_{\mathrm{i}}$ is stable enough to impede the release of the $P_{i}$ from the active site of $M r e B$, the behavior of the $P_{i}$ in each of the three 
simulations of the ADP- $\mathrm{P}_{\mathrm{i}}$-bound state in the presence of A22 (A22+) and in the absence of A22 (A22-) was monitored. One of the trajectories from the A22+ simulations was clustered on the $\mathrm{P}_{\mathrm{i}}$ for a representative snapshot, which is shown in Figure 5.11. The dichlorobenzyl group of A22 remains locked in the hydrophobic pocket formed by the hydrophobic residues, while the isothiourea group interacts with the $\mathrm{P}_{\mathrm{i}}$ via $\mathrm{H}$-bonds.

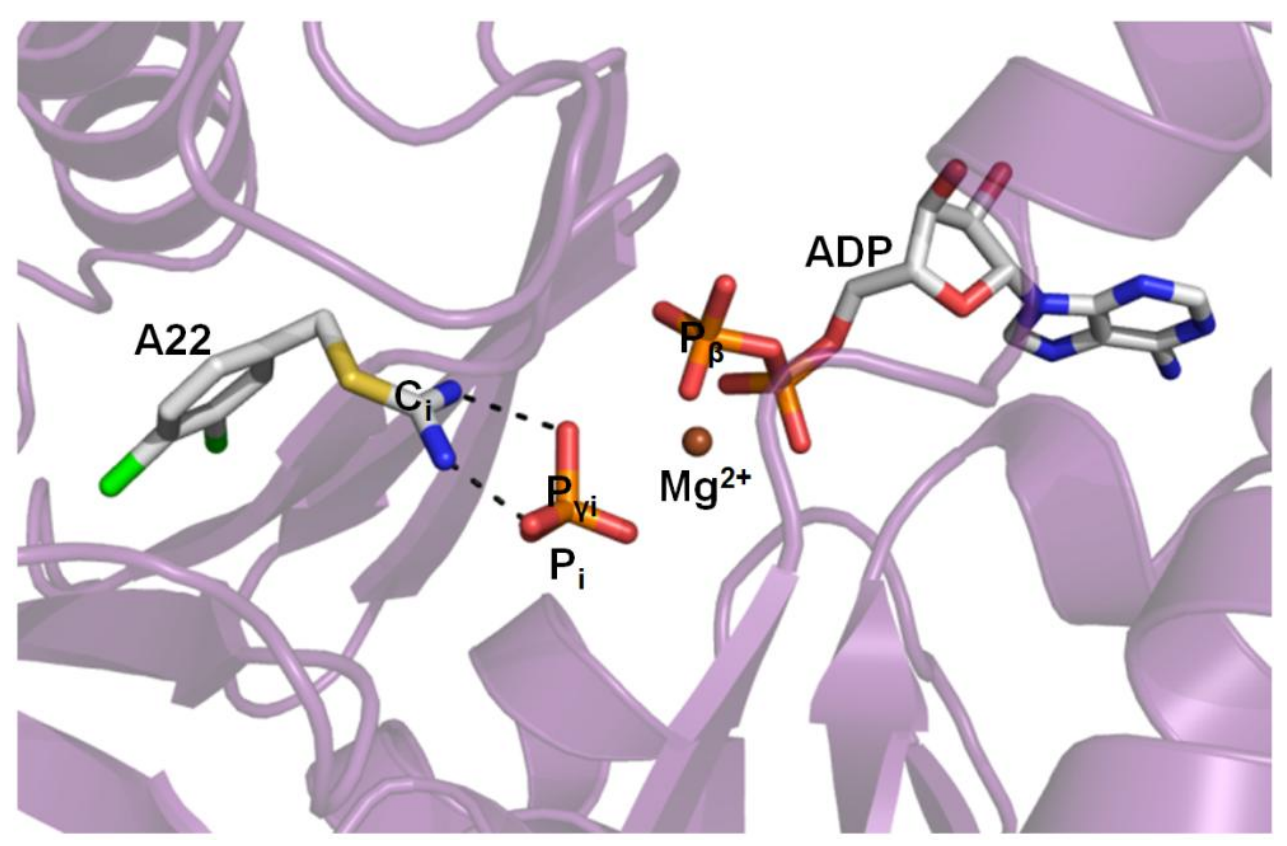

Figure 5.11 Interaction between A22 and the excised $\gamma$-phosphate $\left(\mathrm{P}_{\mathrm{i}}\right)$ of ATP. The Hbonds are indicated by black dashes.

Where applicable, the distances between the phosphorus atom $\left(\mathrm{P}_{\gamma \mathrm{i}}\right)$ of the $\mathrm{P}_{\mathrm{i}}$ and the carbon atom $\left(\mathrm{C}_{\mathrm{i}}\right)$ of the isothiourea group of $\mathrm{A} 22$ and between $\mathrm{P}_{\gamma \mathrm{i}}$ and the phosphorus atom $\left(\mathrm{P}_{\beta}\right)$ of the $\beta$-phosphate of $\mathrm{ADP}$ in the $\mathrm{A} 22+$ and $\mathrm{A} 22-$ simulations were calculated. The latter distance monitors the relative position of the $\mathrm{P}_{\mathrm{i}}$ to the active site. The results are shown in Figure 5.12. The H-bonds formed between A22 and the $\mathrm{P}_{\mathrm{i}}$ keep the distances 
between $\mathrm{P}_{\gamma \mathrm{i}}$ and $\mathrm{C}_{\mathrm{i}}$ (black traces) as well as $\mathrm{P}_{\gamma \mathrm{i}}$ and $\mathrm{P}_{\beta}$ (red traces) relatively constant for at least $55 \mathrm{~ns}$ in the A22+ simulations. This indicates that the $\mathrm{H}$-bonds retard the release of the $\mathrm{P}_{\mathrm{i}}$ by holding it inside the active site. On the contrary, in the A22- simulations, the distance between $\mathrm{P}_{\gamma \mathrm{i}}$ and $\mathrm{P}_{\beta}$ (green traces) increases sharply as a result of the escape of the $\mathrm{P}_{\mathrm{i}}$ out of the active site. These phenomena support the hypothesis that the H-bonds formed between A22 and $\gamma$-phosphate in the ATP-A22-bound state could impede the release of the phosphate from the active site of MreB after ATP hydrolysis.
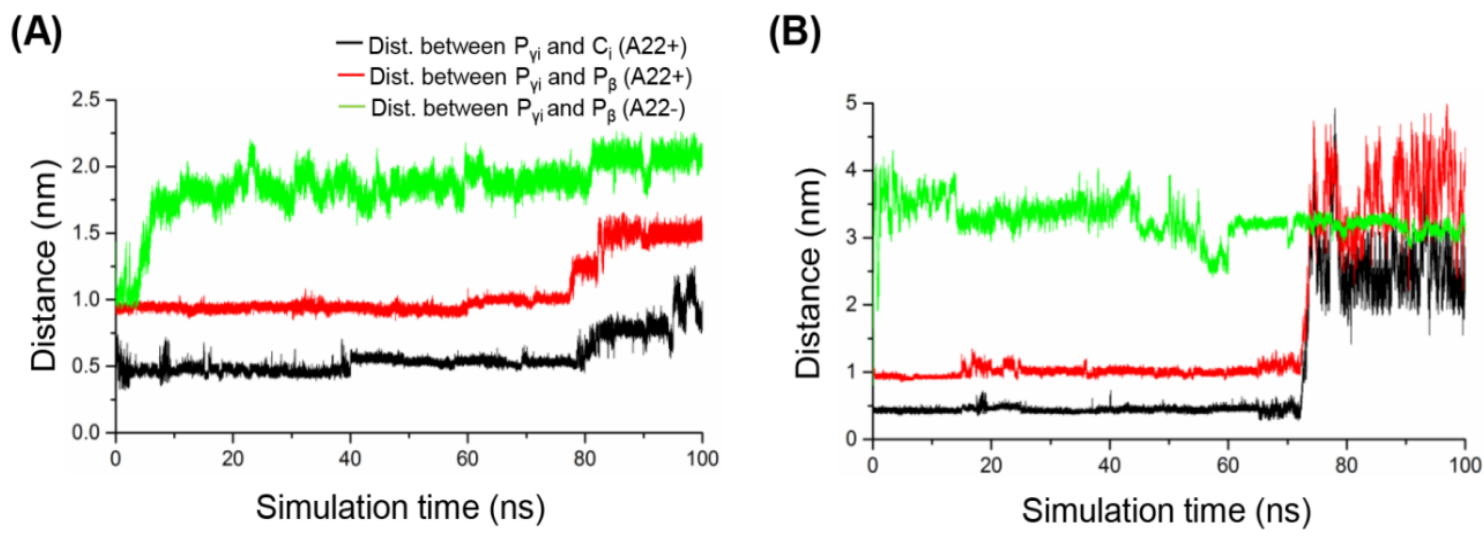

(C)

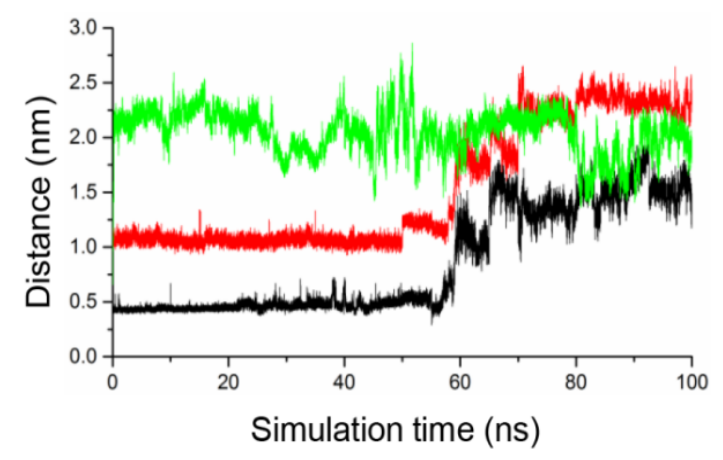

Figure 5.12 Simulation data for the ADP-Pi-bound state of MreB in the presence (A22+) and absence (A22-) of A22. (A-C) Distances between atoms $P_{\gamma i}$ of the $P_{i}$ and $C_{i}$ of $A 22$, and between $\mathrm{P}_{\gamma \mathrm{i}}$ and $\mathrm{P}_{\beta}$ of $\mathrm{ADP}$ in three independent $\mathrm{A} 22+$ and $\mathrm{A} 22$ - simulations. The black, red, and green traces represent the distances between $\mathrm{P}_{\gamma \mathrm{i}}$ and $\mathrm{C}_{\mathrm{i}}$ in the $\mathrm{A} 22+$ simulations, $\mathrm{P}_{\gamma \mathrm{i}}$ and $\mathrm{P}_{\beta}$ in the $\mathrm{A} 22+$ simulations, and $\mathrm{P}_{\gamma \mathrm{i}}$ and $\mathrm{P}_{\beta}$ in the $\mathrm{A} 22-$ simulations, respectively. 


\subsubsection{Proposed mechanism of inhibition of MreB by A22}

The cocrystal structures of MreB bound to ADP/A22 and AMPPNP/MP265 suggested that A22 is not a competitive inhibitor of ATP [238], in contrast with the prediction from a docking study [253]. Here, we show that ATP and A22 are compatible in conjointly binding to MreB in MD simulations. On the basis of the results obtained herein, it is suggested that A22 slows the release of $\mathrm{P}_{\mathrm{i}}$ from the active site of MreB after the hydrolysis of ATP. In this proposed mechanism, in the ATP-A22-bound state of MreB, A22 forms H-bonds with the $\gamma$-phosphate of ATP (Figure 5.3). Therefore, A22 will not affect the catalytic function of Glu140 and the hydrolysis of ATP likely proceeds as normal to generate ADP and $\mathrm{P}_{\mathrm{i}}$. The release of the $\mathrm{P}_{\mathrm{i}}$ from the active site, however, is retarded by the H-bonds it forms with A22 (Figure 5.11).

Furthermore, A22 is predicted to block the $\mathrm{P}_{\mathrm{i}}$ exit pathway, and the consequence of $\mathrm{P}_{\mathrm{i}}$ retention is hypothesized to be the loss of the interprotofillament interactions leading to filament instability [238]. Given the time scale of the MD simulations (100 ns), our data do not address the obstruction of the $\mathrm{P}_{\mathrm{i}}$ exit pathway but do suggest a first step in the delay of $\mathrm{P}_{\mathrm{i}}$ release through direct interaction between A22 and the $\mathrm{P}_{\mathrm{i}}$. A model for the proposed mechanism of delayed phosphate release is illustrated in Figure 5.13. Thus these data indicate an extra element in the slowing of $\mathrm{P}_{\mathrm{i}}$ release, which is predicted to result in filament instability [238]. A22 also increases the critical concentration of MreB for polymerization [253]. This raises two possibilities: either A22 slightly impedes the conformational change in $\mathrm{MreB}$ required to form a filament or the phosphate release is slowed during the formation of the protofilaments preventing the pairing of filaments 
[238] leading to a loss of the effectiveness of the nucleation step. Phosphate release occurs on a similar time scale to polymerization; $[225,234,235]$ however, polymerization can occur in the absence of nucleotide [291], which suggests that A22 may impede the conformational change in MreB that is required to form a filament.

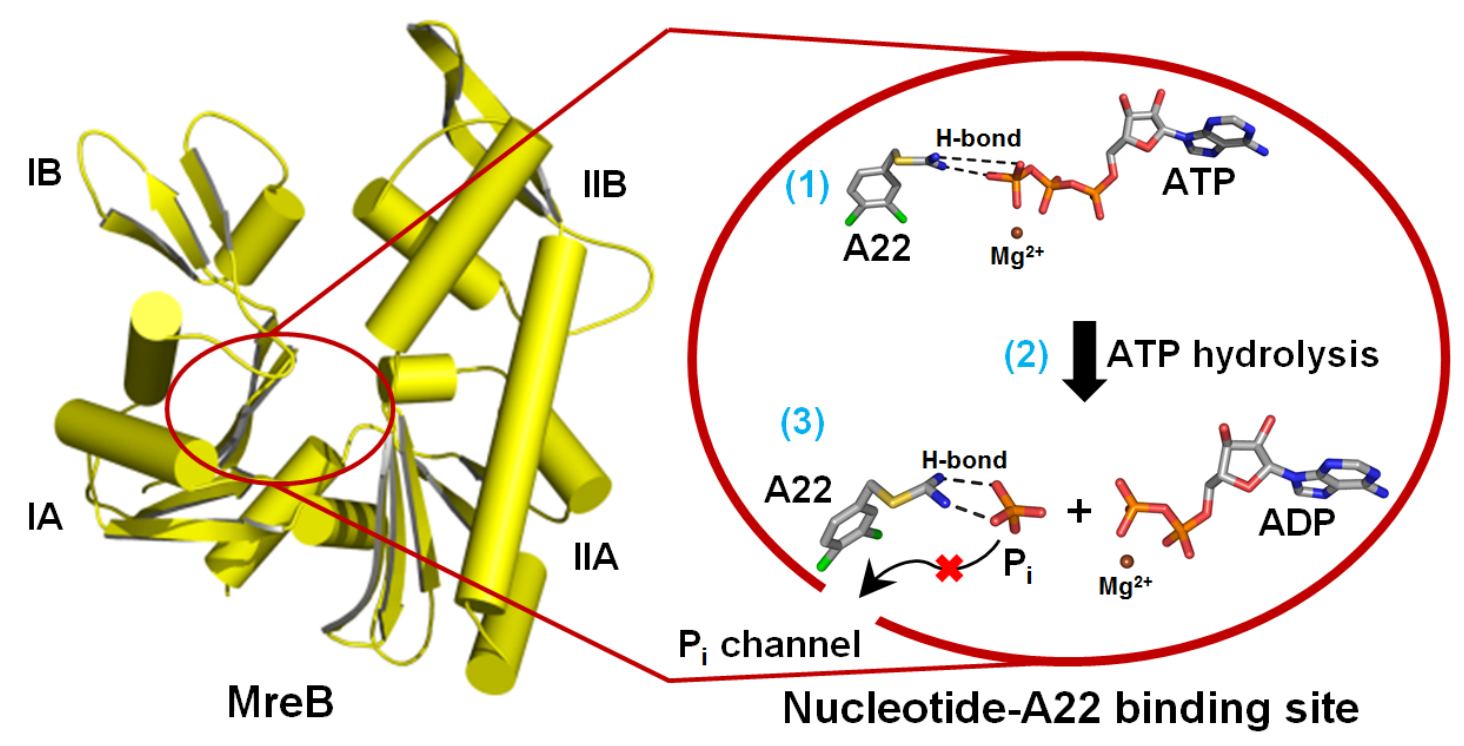

Figure 5.13 Model for the effects of A22. (1) A22 forms H-bonds with the $\gamma$-phosphate of ATP. (2) The free catalytic Glu140 and water molecules trigger the hydrolysis of ATP to generate ADP and $\mathrm{P}_{\mathrm{i}}$. (3) The H-bonds between A22 and $\mathrm{P}_{\mathrm{i}}$ slow the release the release of the $\mathrm{P}_{\mathrm{i}}$. H-bonds are indicated by black dashes.

\subsection{Conclusions}

The simulations revealed that (i) in the ATP/AMPPNP-A22-bound states of MreB, the propensities of A22 for forming $\mathrm{H}$-bonds with the $\gamma$-phosphate groups of ATP and AMPPNP are higher than its propensities for forming H-bonds with the catalytic Glu140 residue, (ii) in the ADP-bound state, the propensity of A22 for forming H-bonds with Glu140 is higher than its propensity for forming H-bonds with the $\beta$-phosphate of ADP, 
and (iii) in the presence of A22, long-lived water molecules at the active site boost ATP hydrolysis. These results explain why ATP hydrolysis can still continue even when A22 binds to MreB, such that the crystal structure of the ATP-A22-bound state has yet to be solved. Our results disagree with the notion that A22 is a competitive inhibitor of ATP [253] and support the idea that both molecules can bind to the NT binding pocket of MreB together [238].

The inhibitory mechanism of A22 is likely to involve the retardation of the release of $\mathrm{P}_{\mathrm{i}}$. This study presents the first simulation study of the dynamics of the NT-A22 binding site of MreB in complex with different NTs and A22 and could be a useful source of information for the design/development of a new generation of more effective inhibitors of MreB. Such inhibitors would be those that could carry out a dual role of inhibiting ATP hydrolysis, by interacting strongly with the catalytic residue or perturbing the docking of water molecules into positions suitable for the hydrolysis and at the same time impeding the release of hydrolyzed phosphate.

\section{NOTE}

The content of this chapter has been published in the Journal of Physical Chemistry B (JPCB). DOI:10.1021/acs.jpcb.6b05199 


\section{Discussion, Conclusions and Future work}

\subsection{Discussion and Conclusions}

The emergence of drug-resistant pathogens calls for the drug discovery industry and stakeholders to step up of efforts to find alternative drugs. Unfortunately, in spite of technological advancement; a better understanding of biochemical systems; detailed understanding of the life cycles, functional proteins and mechanisms of most pathogens, the drug discovery process is still tedious and costly. Drug discovery is characterized by the requirement of a protracted investment of millions of dollars per new molecular entity and often turns out with a low rate of uncovering new pharmaceutical drugs [4-6, 292, 293].

To reduce the cost, lead time, and failures in developing new drugs, computational techniques have been introduced into the prefatory stages of drug discovery. The computational methods are designed to facilitate the testing of a large number of small molecules at a relatively cheaper cost. Unfortunately, however, these computer models apply restraints and approximations to naturally occurring phenomena, such as conformational flexibility of biomolecules, solvation/desolvation, and entropic effects, because of the computational cost involved [108-110, 294, 295]. The overall effect is the generation of false positives/negatives as a result of the oversimplification of the natural biological environment and conditions within which drugs bind to their targets and function. 
The plasticity of proteins and protein-ligand complexes is one of the most important features that are oversimplified by computer-aided drug discovery (CADD) strategies [110, 294, 295]. However, it has been observed that the active/binding sites of proteins are usually located in regions of flexible side chains, loops, and domains to allow for the motions required to accommodate all the stages of biochemical processes $[256,257]$. The active sites of enzymes are usually characterized by relatively high-temperature factors [257]. The flexible nature of active/binding sites of proteins has immense effects on processes such as the active/binding site accessibility, substrate and ligand binding, catalysis, product release, and allosterism [256, 257]. Therefore, accounting for the plasticity of protein receptors and protein-ligand complexes in the CADD process is necessary for identifying true binding processes and gaining useful insights into the mechanisms of drugs or candidate drugs. Such insights could then be carefully incorporated into rational drug design or lead optimization protocols for the development of effective pharmaceutical agents.

The techniques for incorporation protein receptor flexibility into SBVS range from those that consider slight side chain movements to the ones that attempt to include backbone displacements through strategies such as ensemble docking [183]; the more preferred method being docking separately onto MRCs [182]. Currently, the standard practice in ensemble docking is to dock ligands onto multiple conformations of the receptor and then optimize the numerous docking poses to identify the top-scoring pose(s). This strategy fails to deal with the challenge posed by false positives because it simply overlooks the fact that each different binding site conformation introduces a unique set of false positives 
$[172,185,186]$. In this study, conventional MD simulations and molecular docking techniques were applied to develop a ligand selection strategy that makes room for the (a) exploration of the plasticity of the receptor and ligand, (b) exclusion of false positives, and (c) identification of true binders in SBVS. X-ray crystallographic studies involving screening hits and T. maritima MreB were carried out to confirm that the ligand selection strategy can indeed identify true binders. In addition, the A22-MreB interaction was explored in the presence of different NTs through MD simulations. The simulations revealed useful insights about the $\mathrm{A} 22-\mathrm{MreB}$ interaction that could be incorporated in the development of more effective MreB inhibitors.

The ligand selection criteria described herein, which involves identifying the intersection molecules in the top-ranked solutions of all receptors in MRC docking, appears to address the challenge posed by false positives to a larger extent by excluding the false positives from the final selection [282]. In the current state of docking algorithms, the best SBVS protocol can only identify molecules that can bind to the receptor. In the natural biological environment, these binders would have to possess some other important features, which cannot be determined by docking programs, to enable them to reach their targets, escape modification into inactive agents by host enzymes, and avoid ejection by efflux mechanisms. However, since the ability of a drug to bind to the target is an important feature for function, true binders obtained through SBVS could be optimized in lead modification campaigns to meet the desired requirements.

Novelty in structural biology and combinatory chemistry has led to the availability of the structures of many drug targets and the broadening of the chemical space in molecule 
databases respectively, and the evolution of SBVS [296-298]. Nonetheless, the number of pharmaceuticals availed to the drug market has recorded a continuous decline over the last several years [4]. This is partly due to the inefficient use of the databases as a result of placing the focus on only screening hits that have biological activity. To solve this, there is the need for an ideological shift to consider molecules that can at least bind to the target as promising candidate drugs that meet the requirement for selection and optimization. The hit selection criterion described in this study could be useful in this direction as it identifies true binders.

It is worth mentioning that the hit selection strategy described herein does not address some other pertinent concerns in SBVS. In the current state of the protocol, solvation/desolvation and entropic effects are not considered; as is the case in current docking programs. Solvent plays a vital role in ligand binding and stability by mediating interactions between the ligand and the protein receptor. It has been established that entropic and enthalpic contributions by solvent, caused by the displacement of structural water molecules upon ligand binding, improve binding affinity [299, 300]. Thus although it is expensive to incorporate models that quantify solvent effects into docking programs because of their time-demanding nature [299], a complete exclusion of the effects of explicit solvents could have the tendency of creating inconsistencies between docking predictions and experimental observations. Some recent methods take into account the contribution of solvation [299-301], but these are post-docking approaches and would not influence docking prediction and hit determination. The development of docking 
algorithms that could reasonably combine speed and accuracy to explicitly consider entropic and solvation effects in ligand binding could improve enrichment in SBVS.

Another challenge facing the selection strategy is how to determine the clustering RMSD cutoff for selecting structures from the MD trajectory. Currently, we choose a cutoff to ensure that each pair of the representative structures differs by $\geq 0.15 \mathrm{~nm}$ RMSD and that the number of structures does not exceed 10. Thus our choice of a clustering cutoff is greatly influenced by the flexibility of the binding site and the research plan. Arguably, applying the principal component analysis (PCA) method to cluster the trajectory and extracting structures from the local minima in the free energy landscape projections would be the most scientific and acceptable approach. However, we are of the view that selecting structures from the local minima alone would undermine the idea that it is possible for a ligand to bind and stabilize an intermediate structure in the free energy landscape [302]. A total omission of such intermediate conformations may lead to the rejection of some potential inhibitors.

The challenge of accounting for the flexibility of proteins and protein-ligand complexes is not peculiar to docking algorithms. There is evidence that experimentally determined crystal structures poorly quantify the plasticity of some proteins and protein-ligand complexes, and fail to capture intermediate active/binding site conformations [302]. Such intermediate active/binding site conformations could carry the fingerprints required to understand certain biological processes, and could also be trapped and stabilized by appropriate compounds to offer new opportunities for drug discovery [302]. Therefore, the over-reliance on one method for structure determination in the drug discovery process 
could lead to the loss of vital information required for the development of efficient pharmaceuticals.

MD simulation is a powerful tool in the drug discovery process that could be used to explore the plasticity of the protein receptor and protein-ligand complex. In the study of the dynamics of the crystallographic structures of MreB-A22 complexes in the presence of different NTs by MD simulations herein, vital information that is missing in the experimental structures but could be useful in the development of a new generation of anti-MreB drugs was obtained. This information includes details about the A22-MreB interaction pattern, details about the dynamics of water and the effect on ATP hydrolysis, and details about the effect of A22 on $\mathrm{P}_{\mathrm{i}}$ release. Thus the combination of structural information from both experimental and computer-generated structures is required to provide a better synergy to facilitate the discovery and design of promising drugs.

\subsection{Future work}

A docking simulation study that is capable of identifying true binders in SBVS when receptor plasticity is considered has been described and validated in this study. Even though three molecules (A1, B2, and C3) that could bind to the active site of MreB were found, preliminary in vitro bioactivity (killing) assays on E. coli showed that these molecules are less effective than A22. The structural properties of the three compounds suggest that poor pharmacokinetic properties could be responsible for their weak bactericidal effects. 
Considering the fact that MreB is located in the cytosol of the bacterial cell, effective plasma membrane permeability is a feature every potential MreB inhibitor should possess. The ability of a molecule to traverse the hydrophobic core of the plasma membrane could be roughly estimated by its $\operatorname{cLogP}$ value, which is the logarithm of the ratio of its concentration in $\mathrm{n}$-octanol to its concentration in water. The higher the $\operatorname{cLogP}$ of a molecule, the more hydrophobic it is, and the better its chances of traversing the plasma membrane. The cLogP values of the three MreB binders are within a range of 1.71- 2.31, making them more hydrophilic than A22 which has a $\operatorname{cLogP}$ value of 2.95. The hydrophilic nature of these molecules could be a factor that impedes their ability to permeate the bacterial membrane to bind and inhibit MreB in vivo. This could be a reason, among other possible reasons, why the three compounds were less effective than A22 in the E. coli killing assays. Hence, a future study could embark on a lead optimization campaign aimed at improving the pharmacological properties of these molecules to make them more effective against of MreB.

Furthermore, in the MD simulations study of the dynamics of the active site of MreB in complex with A22 and different NTs, it was observed that one possible reason why A22 is not as effective as commercially available antibiotics is because it may not be an inhibitor of ATP hydrolysis. It was also observed that A22 fails to (a) interact with the catalytic Glu140 residue and (b) disorient lytic water molecules in the presence of ATP. To find a more potent antibiotic against $\mathrm{MreB}$, it is desirable that a future study incorporates these ideas in the design of drugs that could inhibit the hydrolysis of ATP by interacting 
strongly with Glu140 and at the same time perturb the proper localization of lytic water molecules in the catalytic site. 


\section{Appendix A}

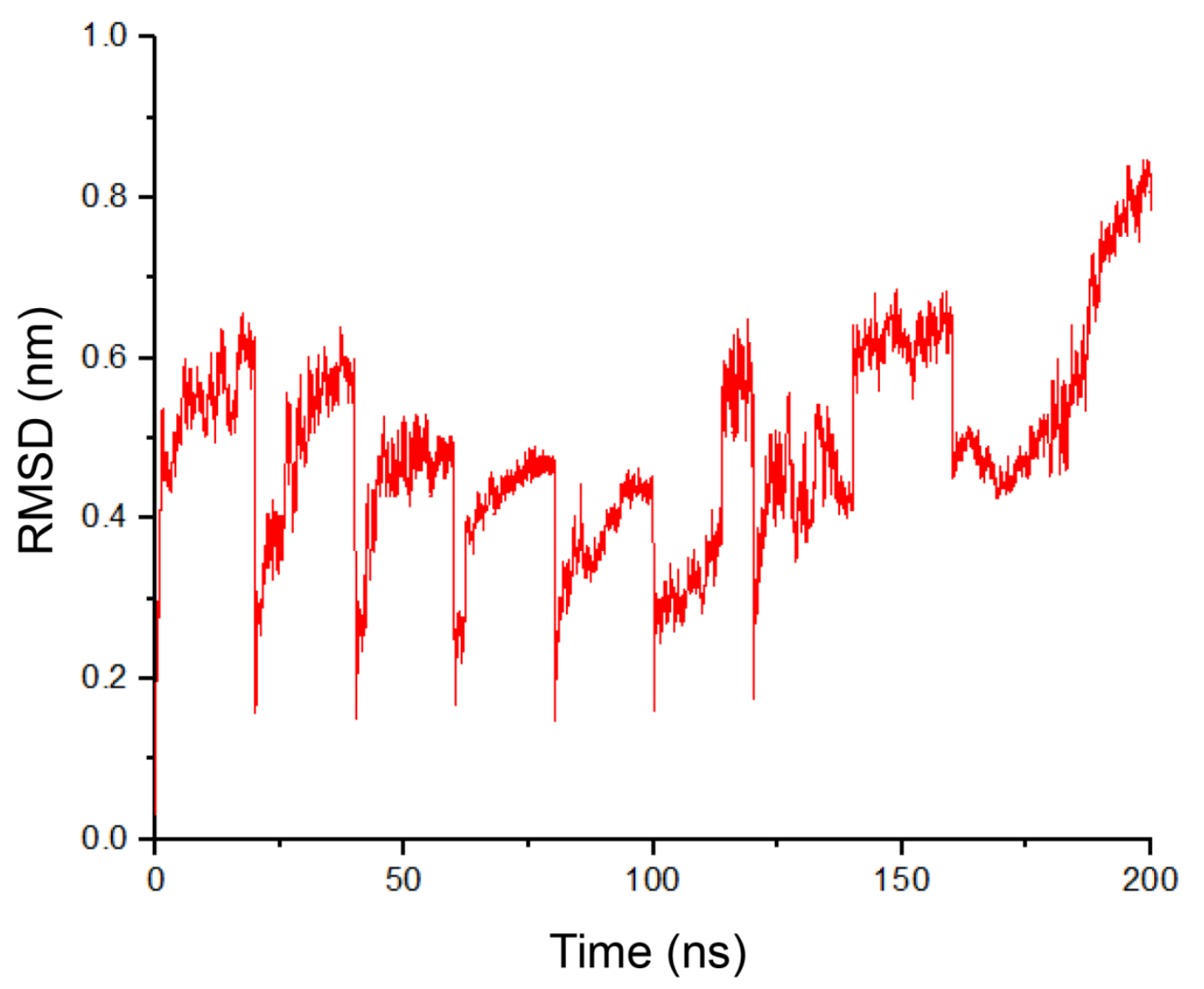

Figure A.1RMSD of NP backbone in the 200 ns net trajectory used for clustering. The first 100 ns show the RMSD in the first five independent simulations of the crystal structure. The last 100 ns show the RMSD in the independent simulations of the final structures of the first five simulations. 
Table A.1 Number of intersection molecules in different number of conformations of the T-loop binding pocket and RNA binding site of NP at different levels of comparison.

\begin{tabular}{|c|c|c|c|c|c|}
\hline & \multicolumn{5}{|c|}{ Intersection Molecules for the T-Loop Binding Pocket } \\
\hline \multicolumn{6}{|c|}{ No. of Conformations } \\
\hline Level & $>1$ & $>2$ & $>3$ & $>4$ & $>5$ \\
\hline Top 50 & 81 & 34 & 16 & 4 & 1 \\
\hline Top 100 & 162 & 96 & 50 & 19 & 3 \\
\hline Top 150 & 240 & 158 & 92 & 38 & 6 \\
\hline Top 200 & 293 & 225 & 140 & 77 & 14 \\
\hline \multicolumn{6}{|c|}{ Intersection Molecules for the RNA Binding Site } \\
\hline \multicolumn{6}{|c|}{ No. of Conformations } \\
\hline Level & $>1$ & $>2$ & $>3$ & $>4$ & \\
\hline Top 10 & 10 & 2 & 0 & 0 & \\
\hline Top 20 & 22 & 8 & 2 & 1 & \\
\hline Top 30 & 31 & 14 & 6 & 2 & \\
\hline Top 40 & 42 & 20 & 10 & 3 & \\
\hline Top 50 & 56 & 28 & 13 & 7 & \\
\hline
\end{tabular}

Table A.2 Chemical name of molecules selected for crystallographic studies with MreB.

\begin{tabular}{|c|c|}
\hline Molecule & Chemical name \\
\hline $\mathrm{A} 1$ & $\begin{array}{l}\text { 2-(\{[amino(imino)methyl]thio }\} \text { methyl)-4,5-dimethylbenzyl } \\
\text { aminomethanimidothioate }\end{array}$ \\
\hline B2 & N-Hydroxy-2-[4-(trifluoromethyl)phenoxy]ethanimidamide \\
\hline $\mathrm{C} 3$ & 3-(\{[amino(imino)methyl]thio $\}$ methyl)-2-fluorobenzyl aminomethanimidothioate \\
\hline D4 & $1-\{[($ aminocarbohydrazonoyl)thio $]$ methyl $\}-4$-methylbenzene \\
\hline E5 & 2-[(3,4-dichlorophenyl)methylene]-1-hydrazinecarboximidamide \\
\hline F6 & 3-Chloro-1-benzothiophene-2-carbohydrazide \\
\hline G7 & 4-hydrazinopyrido[3',2':4,5]thieno[3,2-d]pyrimidine \\
\hline H8 & 4-(1,2,3-thiadiazol-4-yl)benzyl aminomethanimidothioate \\
\hline I9 & (\{ imino[(4-phenyl-1,3-thiazol-2-yl)amino]methyl $\}$ amino)methanimidamide \\
\hline $\mathrm{J} 10$ & N-hydrazino(imino)methyl-N'-mesitylguanidine \\
\hline K11 & N-(4-Bromo-3-methylphenyl)hydrazinecarbothioamide \\
\hline
\end{tabular}




\section{References}

1. Jones, A.W., Early Drug Discovery and the Rise of Pharmaceutical Chemistry. Drug Testing and Analysis, 2011. 3(6): p. 337-344.

2. Klockgether-Radke, A.P., F. W. Serturner and the Discovery of Morphine. 200 Years of Pain Therapy with Opioids. Anasthesiologie Intensivmedizin Notfallmedizin Schmerztherapie, 2002. 37(5): p. 244-249.

3. Sneader, W., Drug Discovery. A History. 2005, New York: John Wiley \& Sons Ltd. Pp. 1-234.

4. Paul, S.M., D.S. Mytelka, C.T. Dunwiddie, C.C. Persinger, B.H. Munos, S.R. Lindborg, and A.L. Schacht, How to Improve R\&D Productivity: The Pharmaceutical Industry's Grand Challenge. Nature Reviews Drug Discovery, 2010. 9(3): p. 203-214.

5. DiMasi, J.A., R.W. Hansen, and H.G. Grabowski, The Price of Innovation: New Estimates of Drug Development Costs. Journal of Health Economics, 2003. 22(2): p. 151-185.

6. Song, C.M., S.J. Lim, and J.C. Tong, Recent Advances in Computer-Aided Drug Design. Briefings in Bioinformatics, 2009. 10(5): p. 579-591.

7. Katsila, T., G.A. Spyroulias, G.P. Patrinos, and M.-T. Matsoukas, Computational Approaches in Target Identification and Drug Discovery. Computational and Structural Biotechnology Journal, 2016. 14: p. 177-184.

8. Chen, Y.P.P. and F. Chen, Identifying Targets for Drug Discovery Using Bioinformatics. Expert Opinion on Therapeutic Targets, 2008. 12(4): p. 383-389.

9. Wang, S.L., T.B. Sim, and Y.T. Chang, Tools for Target Identification and Validation. Current Opinion in Chemical Biology, 2004. 8(4): p. 371-377. 
10. Yang, Y.L., S.J. Adelstein, and A.I. Kassis, Target Discovery from Data Mining Approaches. Drug Discovery Today, 2009. 14(3-4): p. 147-154.

11. Smith, C., Drug Target Validation: Hitting the Target. Nature, 2003. 422(6929): p. 341-347.

12. Jones, L.H., An Industry Perspective on Drug Target Validation. Expert Opinion on Drug Discovery, 2016. 11(7): p. 623-625.

13. Scherer, L.J. and J.J. Rossi, Approaches for the Sequence-Specific Knockdown of Mrna. Nature Biotechnology, 2003. 21(12): p. 1457-1465.

14. Couzin, J., Small Rnas Make Big Splash. Science, 2002. 298(5602): p. 2296-2297.

15. Hannon, G.J., RNA Interference. Nature, 2002. 418(6894): p. 244-251.

16. Agami, R., Rnai and Related Mechanisms and Their Potential Use for Therapy. Current Opinion in Chemical Biology, 2002. 6(6): p. 829-834.

17. Milhavet, O., D.S. Gary, and M.P. Mattson, RNA Interference in Biology and Medicine. Pharmacological Reviews, 2003. 55(4): p. 629-648.

18. Kurreck, J., Antisense Technologies - Improvement through Novel Chemical Modifications. European Journal of Biochemistry, 2003. 270(8): p. 1628-1644.

19. Kopec, K.K., D. Bozyczko-Coyne, and M. Williams, Target Identification and Validation in Drug Discovery: The Role of Proteomics. Biochemical Pharmacology, 2005. 69(8): p. 1133-1139.

20. Halgren, T.A., Identifying and Characterizing Binding Sites and Assessing Druggability. Journal of Chemical Information and Modeling, 2009. 49(2): p. 377389. 
21. Nayal, M. and B. Honig, On the Nature of Cavities on Protein Surfaces: Application to the Identification of Drug-Binding Sites. Proteins-Structure Function and Bioinformatics, 2006. 63(4): p. 892-906.

22. Seco, J., F.J. Luque, and X. Barril, Binding Site Detection and Druggability Index from First Principles. Journal of Medicinal Chemistry, 2009. 52(8): p. 2363-2371.

23. Bakan, A., N. Nevins, A.S. Lakdawala, and I. Bahar, Druggability Assessment of Allosteric Proteins by Dynamics Simulations in the Presence of Probe Molecules. Journal of Chemical Theory and Computation, 2012. 8(7): p. 2435-2447.

24. Gupta, A., A.K. Gupta, and K. Seshadri, Structural Models in the Assessment of Protein Druggability Based on Hts Data. Journal of Computer-Aided Molecular Design, 2009. 23(8): p. 583-592.

25. Schmidtke, P. and X. Barril, Understanding and Predicting Druggability. A HighThroughput Method for Detection of Drug Binding Sites. Journal of Medicinal Chemistry, 2010. 53(15): p. 5858-5867.

26. Bowie, J.U., R. Luthy, and D. Eisenberg, A Method to Identify Protein Sequences That Fold into a Known 3-Dimensional Structure. Science, 1991. 253(5016): p. 164-170.

27. Dror, O., A. Shulman-Peleg, R. Nussinov, and H.J. Wolfson, Predicting Molecular Interactions in Silico: I. A Guide to Pharmacophore Identification and Its Applications to Drug Design. Current Medicinal Chemistry, 2004. 11(1): p. 71-90.

28. Jahn, A., G. Hinselmann, N. Fechner, and A. Zell, Optimal Assignment Methods for Ligand-Based Virtual Screening. Journal of Cheminformatics, 2009. 1(1): p. 14.

29. Martin, Y.C., J.L. Kofron, and L.M. Traphagen, Do Structurally Similar Molecules Have Similar Biological Activity? Journal of Medicinal Chemistry, 2002. 45(19): p. 4350-4358. 
30. Bender, A., J.L. Jenkins, J. Scheiber, S.C.K. Sukuru, M. Glick, and J.W. Davies, How Similar Are Similarity Searching Methods? A Principal Component Analysis of Molecular Descriptor Space. Journal of Chemical Information and Modeling, 2009. 49(1): p. 108-119.

31. Sheridan, R.P. and S.K. Kearsley, Why Do We Need So Many Chemical Similarity Search Methods? Drug Discovery Today, 2002. 7(17): p. 903-911.

32. Villoutreix, B.O., N. Renault, D. Lagorce, O. Sperandio, M. Montes, and M.A. Miteva, Free Resources to Assist Structure-Based Virtual Ligand Screening Experiments. Current Protein \& Peptide Science, 2007. 8(4): p. 381-411.

33. Kitchen, D.B., H. Decornez, J.R. Furr, and J. Bajorath, Docking and Scoring in Virtual Screening for Drug Discovery: Methods and Applications. Nature Reviews Drug Discovery, 2004. 3(11): p. 935-949.

34. Brooijmans, N. and I.D. Kuntz, Molecular Recognition and Docking Algorithms. Annual Review of Biophysics and Biomolecular Structure, 2003. 32: p. 335-373.

35. McGaughey, G.B., R.P. Sheridan, C.I. Bayly, J.C. Culberson, C. Kreatsoulas, S. Lindsley, V. Maiorov, J.F. Truchon, and W.D. Cornell, Comparison of Topological, Shape, and Docking Methods in Virtual Screening. Journal of Chemical Information and Modeling, 2007. 47(4): p. 1504-1519.

36. Wei, D.Q., R. Zhang, Q.S. Du, W.N. Gao, Y. Li, H. Gao, S.Q. Wang, X. Zhang, A.X. Li, S. Sirois, and K.C. Chou, Anti-Sars Drug Screening by Molecular Docking. Amino Acids, 2006. 31(1): p. 73-80.

37. Barreiro, G., C.R.W. Guimaraes, I. Tubert-Brohman, T.M. Lyons, J. Tirado-Rives, and W.L. Jorgensen, Search for Non-Nucleoside Inhibitors of Hiv-1 Reverse Transcriptase Using Chemical Similarity, Molecular Docking, and Mm-Gb/Sa Scoring. Journal of Chemical Information and Modeling, 2007. 47(6): p. 24162428. 
38. Tikhonova, I.G., C.S. Sum, S. Neumann, S. Engel, B.M. Raaka, S. Costanzi, and M.C. Gershengorn, Discovery of Novel Agonists and Antagonists of the Free Fatty Acid Receptor 1 (Ffar1) Using Virtual Screening. Journal of Medicinal Chemistry, 2008. 51(3): p. 625-633.

39. Vidal, D., M. Thormann, and M. Pons, A Novel Search Engine for Virtual Screening of Very Large Databases. Journal of Chemical Information and Modeling, 2006. 46(2): p. 836-843.

40. Mestres, J. and R.M.A. Knegtel, Similarity Versus Docking in 3d Virtual Screening. Perspectives in Drug Discovery and Design, 2000. 20(1): p. 191-207.

41. Bissantz, C., C. Schalon, W. Guba, and M. Stahl, Focused Library Design in Gpcr Projects on the Example of 5-Ht2c Agonists: Comparison of Structure-Based Virtual Screening with Ligand-Based Search Methods. Proteins-Structure Function and Bioinformatics, 2005. 61(4): p. 938-952.

42. Reynolds, C.H., Impact of Computational Structure-Based Methods on Drug Discovery. Current Pharmaceutical Design, 2014. 20(20): p. 3380-3386.

43. Shoichet, B.K., S.L. McGovern, B.Q. Wei, and J.J. Irwin, Lead Discovery Using Molecular Docking. Current Opinion in Chemical Biology, 2002. 6(4): p. 439-446.

44. Villoutreix, B.O., R. Eudes, and M.A. Miteva, Structure-Based Virtual Ligand Screening: Recent Success Stories. Combinatorial Chemistry \& High Throughput Screening, 2009. 12(10): p. 1000-1016.

45. Seifert, M.H.J. and M. Lang, Essential Factors for Successful Virtual Screening. Mini-Reviews in Medicinal Chemistry, 2008. 8(1): p. 63-72.

46. Sousa, S.F., P.A. Fernandes, and M.J. Ramos, Protein-Ligand Docking: Current Status and Future Challenges. Proteins-Structure Function and Bioinformatics, 2006. 65(1): p. 15-26. 
47. Goodsell, D.S., G.M. Morris, and A.J. Olson, Automated Docking of Flexible Ligands: Applications of Autodock. Journal of Molecular Recognition, 1996. 9(1): p. $1-5$.

48. Jones, G., P. Willett, R.C. Glen, A.R. Leach, and R. Taylor, Development and Validation of a Genetic Algorithm for Flexible Docking. Journal of Molecular Biology, 1997. 267(3): p. 727-748.

49. Ewing, T.J.A., S. Makino, A.G. Skillman, and I.D. Kuntz, Dock 4.0: Search Strategies for Automated Molecular Docking of Flexible Molecule Databases. Journal of Computer-Aided Molecular Design, 2001. 15(5): p. 411-428.

50. Rarey, M., B. Kramer, T. Lengauer, and G. Klebe, A Fast Flexible Docking Method Using an Incremental Construction Algorithm. Journal of Molecular Biology, 1996. 261(3): p. 470-489.

51. Friesner, R.A., J.L. Banks, R.B. Murphy, T.A. Halgren, J.J. Klicic, D.T. Mainz, M.P. Repasky, E.H. Knoll, M. Shelley, J.K. Perry, D.E. Shaw, P. Francis, and P.S. Shenkin, Glide: A New Approach for Rapid, Accurate Docking and Scoring. 1. Method and Assessment of Docking Accuracy. Journal of Medicinal Chemistry, 2004. 47(7): p. 1739-1749.

52. Halgren, T.A., R.B. Murphy, R.A. Friesner, H.S. Beard, L.L. Frye, W.T. Pollard, and J.L. Banks, Glide: A New Approach for Rapid, Accurate Docking and Scoring. 2. Enrichment Factors in Database Screening. Journal of Medicinal Chemistry, 2004. 47(7): p. 1750-1759.

53. Jones, G., P. Willett, and R.C. Glen, Molecular Recognition of Receptor-Sites Using a Genetic Algorithm with a Description of Desolvation. Journal of Molecular Biology, 1995. 245(1): p. 43-53.

54. Sousa, S.F., A.J.M. Ribeiro, J.T.S. Coimbra, R.P.P. Neves, S.A. Martins, N.S.H.N. Moorthy, P.A. Fernandes, and M.J. Ramos, Protein-Ligand Docking in the New 
Millennium - a Retrospective of 10 Years in the Field. Current Medicinal Chemistry, 2013. 20(18): p. 2296-2314.

55. Ewing, T.J.A. and I.D. Kuntz, Critical Evaluation of Search Algorithms for Automated Molecular Docking and Database Screening. Journal of Computational Chemistry, 1997. 18(9): p. 1175-1189.

56. Lang, P.T., S.R. Brozell, S. Mukherjee, E.F. Pettersen, E.C. Meng, V. Thomas, R.C. Rizzo, D.A. Case, T.L. James, and I.D. Kuntz, Dock 6: Combining Techniques to Model RNA-Small Molecule Complexes. Rna-a Publication of the Rna Society, 2009. 15(6): p. 1219-1230.

57. Goodsell, D.S. and A.J. Olson, Automated Docking of Substrates to Proteins by Simulated Annealing. Proteins-Structure Function and Genetics, 1990. 8(3): p. 195-202.

58. Morris, G.M., D.S. Goodsell, R.S. Halliday, R. Huey, W.E. Hart, R.K. Belew, and A.J. Olson, Automated Docking Using a Lamarckian Genetic Algorithm and an Empirical Binding Free Energy Function. Journal of Computational Chemistry, 1998. 19(14): p. 1639-1662.

59. Morris, G.M., D.S. Goodsell, R. Huey, and A.J. Olson, Distributed Automated Docking of Flexible Ligands to Proteins: Parallel Applications of Autodock 2.4. Journal of Computer-Aided Molecular Design, 1996. 10(4): p. 293-304.

60. Jiang, F. and S.H. Kim, Soft Docking - Matching of Molecular-Surface Cubes. Journal of Molecular Biology, 1991. 219(1): p. 79-102.

61. Hart, T.N. and R.J. Read, A Multiple-Start Monte-Carlo Docking Method. Proteins-Structure Function and Genetics, 1992. 13(3): p. 206-222.

62. Bohm, H.J., The Computer-Program Ludi - a New Method for the Denovo Design of Enzyme-Inhibitors. Journal of Computer-Aided Molecular Design, 1992. 6(1): p. 61-78. 
63. Mizutani, M.Y., N. Tomioka, and A. Itai, Rational Automatic Search Method for Stable Docking Models of Protein and Ligand. Journal of Molecular Biology, 1994. 243(2): p. 310-326.

64. Miller, M.D., S.K. Kearsley, D.J. Underwood, and R.P. Sheridan, Flog - a System to Select Quasi-Flexible Ligands Complementary to a Receptor of Known 3Dimensional Structure. Journal of Computer-Aided Molecular Design, 1994. 8(2): p. 153-174.

65. Pang, Y.P. and A.P. Kozikowski, Prediction of the Binding-Site of 1-Benzyl-4(5,6-Dimethoxy-1-Indanon-2-Yl)Methyl Piperidine in Acetylcholinesterase by Docking Studies with the Sysdoc Program. Journal of Computer-Aided Molecular Design, 1994. 8(6): p. 683-693.

66. Clark, K.P. and Ajay, Flexible Ligand Docking without Parameter Adjustment across 4 Ligand-Receptor Complexes. Journal of Computational Chemistry, 1995. 16(10): p. 1210-1226.

67. Kramer, B., M. Rarey, and T. Lengauer, Evaluation of the Flexx Incremental Construction Algorithm for Protein-Ligand Docking. Proteins-Structure Function and Genetics, 1999. 37(2): p. 228-241.

68. Welch, W., J. Ruppert, and A.N. Jain, Hammerhead: Fast, Fully Automated Docking of Flexible Ligands to Protein Binding Sites. Chemistry \& Biology, 1996. 3(6): p. 449-462.

69. Sobolev, V., R.C. Wade, G. Vriend, and M. Edelman, Molecular Docking Using Surface Complementarity. Proteins-Structure Function and Genetics, 1996. 25(1): p. $120-129$.

70. Gabb, H.A., R.M. Jackson, and M.J.E. Sternberg, Modelling Protein Docking Using Shape Complementarity, Electrostatics and Biochemical Information. Journal of Molecular Biology, 1997. 272(1): p. 106-120. 
71. Totrov, M. and R. Abagyan, Flexible Protein-Ligand Docking by Global Energy Optimization in Internal Coordinates. Proteins-Structure Function and Genetics, 1997: p. 215-220.

72. McMartin, C. and R.S. Bohacek, Qxp: Powerful, Rapid Computer Algorithms for Structure-Based Drug Design. Journal of Computer-Aided Molecular Design, 1997. 11(4): p. 333-344.

73. Baxter, C.A., C.W. Murray, D.E. Clark, D.R. Westhead, and M.D. Eldridge, Flexible Docking Using Tabu Search and an Empirical Estimate of Binding Affinity. Proteins-Structure Function and Genetics, 1998. 33(3): p. 367-382.

74. Burkhard, P., P. Taylor, and M.D. Walkinshaw, An Example of a Protein Ligand Found by Database Mining: Description of the Docking Method and Its Verification by a 2.3 Angstrom X-Ray Structure of a Thrombin-Ligand Complex. Journal of Molecular Biology, 1998. 277(2): p. 449-466.

75. Liu, M. and S.M. Wang, Mcdock: A Monte Carlo Simulation Approach to the Molecular Docking Problem. Journal of Computer-Aided Molecular Design, 1999. 13(5): p. 435-451.

76. Trosset, J.Y. and H.A. Scheraga, Prodock: Software Package for Protein Modeling and Docking. Journal of Computational Chemistry, 1999. 20(4): p. 412-427.

77. Taylor, J.S. and R.M. Burnett, Darwin: A Program for Docking Flexible Molecules. Proteins-Structure Function and Genetics, 2000. 41(2): p. 173-191.

78. Pang, Y.P., E. Perola, K. Xu, and F.G. Prendergast, Eudoc: A Computer Program for Identification of Drug Interaction Sites in Macromolecules and Drug Leads from Chemical Databases. Journal of Computational Chemistry, 2001. 22(15): p. 1750-1771. 
79. Claussen, H., C. Buning, M. Rarey, and T. Lengauer, Flexe: Efficient Molecular Docking Considering Protein Structure Variations. Journal of Molecular Biology, 2001. 308(2): p. 377-395.

80. Taylor, R.D., P.J. Jewsbury, and J.W. Essex, Fds: Flexible Ligand and Receptor Docking with a Continuum Solvent Model and Soft-Core Energy Function. Journal of Computational Chemistry, 2003. 24(13): p. 1637-1656.

81. McGann, M.R., H.R. Almond, A. Nicholls, J.A. Grant, and F.K. Brown, Gaussian Docking Functions. Biopolymers, 2003. 68(1): p. 76-90.

82. Venkatachalam, C.M., X. Jiang, T. Oldfield, and M. Waldman, Ligandfit: A Novel Method for the Shape-Directed Rapid Docking of Ligands to Protein Active Sites. Journal of Molecular Graphics \& Modelling, 2003. 21(4): p. 289-307.

83. Joseph-McCarthy, D., B.E. Thomas, M. Belmarsh, D. Moustakas, and J.C. Alvarez, Pharmacophore-Based Molecular Docking to Account for Ligand Flexibility. Proteins-Structure Function and Genetics, 2003. 51(2): p. 172-188.

84. Jain, A.N., Surflex: Fully Automatic Flexible Molecular Docking Using a Molecular Similarity-Based Search Engine. Journal of Medicinal Chemistry, 2003. 46(4): p. 499-511.

85. Yang, J.M. and C.C. Chen, Gemdock: A Generic Evolutionary Method for Molecular Docking. Proteins-Structure Function and Bioinformatics, 2004. 55(2): p. 288-304.

86. Seifert, M.H.J., F. Schmitt, T. Herz, and B. Kramer, Propose: A Docking Engine Based on a Fully Configurable Protein-Ligand Interaction Model. Journal of Molecular Modeling, 2004. 10(5-6): p. 342-357.

87. Choi, V., Yucca: An Efficient Algorithm for Small-Molecule Docking. Chemistry \& Biodiversity, 2005. 2(11): p. 1517-1524. 
88. Thomsen, R. and M.H. Christensen, Moldock: A New Technique for HighAccuracy Molecular Docking. Journal of Medicinal Chemistry, 2006. 49(11): p. 3315-3321.

89. Korb, O., T. Stutzle, and T.E. Exner, Plants: Application of Ant Colony Optimization to Structure-Based Drug Design, in Ant Colony Optimization and Swarm Intelligence, Proceedings, M. Dorigo, et al., Editors. 2006. p. 247-258.

90. Pei, J.F., Q. Wang, Z.M. Liu, Q.L. Li, K. Yang, and L.H. Lai, Psi-Dock: Towards Highly Efficient and Accurate Flexible Ligand Docking. Proteins-Structure Function and Bioinformatics, 2006. 62(4): p. 934-946.

91. Zsoldos, Z., D. Reid, A. Simon, S.B. Sadjad, and A.P. Johnson, Ehits: A New Fast, Exhaustive Flexible Ligand Docking System. Journal of Molecular Graphics \& Modelling, 2007. 26(1): p. 198-212.

92. Grosdidier, A., V. Zoete, and O. Michielin, Eadock: Docking of Small Molecules into Protein Active Sites with a Multiobjective Evolutionary Optimization. Proteins-Structure Function and Bioinformatics, 2007. 67(4): p. 1010-1025.

93. Zhao, Y. and M.F. Sanner, Flipdock: Docking Flexible Ligands into Flexible Receptors. Proteins-Structure Function and Bioinformatics, 2007. 68(3): p. 726737.

94. Huang, S.-Y. and X. Zou, Ensemble Docking of Multiple Protein Structures: Considering Protein Structural Variations in Molecular Docking. ProteinsStructure Function and Bioinformatics, 2007. 66(2): p. 399-421.

95. Namasivayam, V. and R. Guenther, Pso@Autodock: A Fast Flexible Molecular Docking Program Based on Swarm Intelligence. Chemical Biology \& Drug Design, 2007. 70(6): p. 475-484. 
96. Gupta, A., A. Gandhimathi, P. Sharma, and B. Jayaram, Pardock: An All Atom Energy Based Monte Carlo Docking Protocol for Protein-Ligand Complexes. Protein and Peptide Letters, 2007. 14(7): p. 632-646.

97. Chen, H.-M., B.-F. Liu, H.-L. Huang, S.-F. Hwang, and S.-Y. Ho, Sodock: Swarm Optimization for Highly Flexible Protein-Ligand Docking. Journal of Computational Chemistry, 2007. 28(2): p. 612-623.

98. Stroganov, O.V., F.N. Novikov, V.S. Stroylov, V. Kulkov, and G.G. Chilov, Lead Finder: An Approach to Improve Accuracy of Protein-Ligand Docking, Binding Energy Estimation, and Virtual Screening. Journal of Chemical Information and Modeling, 2008. 48(12): p. 2371-2385.

99. Sauton, N., D. Lagorce, B.O. Villoutreix, and M.A. Miteva, Ms-Dock: Accurate Multiple Conformation Generator and Rigid Docking Protocol for Multi-Step Virtual Ligand Screening. Bmc Bioinformatics, 2008. 9.

100. Brylinski, M. and J. Skolnick, Q-Dock: Low-Resolution Flexible Ligand Docking with Pocket-Specific Threading Restraints. Journal of Computational Chemistry, 2008. 29(10): p. 1574-1588.

101. Cerqueira, N.M.F.S.A., N.F. Bras, P.A. Fernandes, and M.J. Ramos, Madamm: A Multistaged Docking with an Automated Molecular Modeling Protocol. ProteinsStructure Function and Bioinformatics, 2009. 74(1): p. 192-206.

102. Trott, O. and A.J. Olson, Software News and Update Autodock Vina: Improving the Speed and Accuracy of Docking with a New Scoring Function, Efficient Optimization, and Multithreading. Journal of Computational Chemistry, 2010. 31(2): p. 455-461.

103. Singh, T., D. Biswas, and B. Jayaram, Aads - an Automated Active Site Identification, Docking, and Scoring Protocol for Protein Targets Based on 
Physicochemical Descriptors. Journal of Chemical Information and Modeling, 2011. 51(10): p. 2515-2527.

104. Kim, D.-S., C.-M. Kim, C.-I. Won, J.-K. Kim, J. Ryu, Y. Cho, C. Lee, and J. Bhak, Betadock: Shape-Priority Docking Method Based on Beta-Complex. Journal of Biomolecular Structure \& Dynamics, 2011. 29(1): p. 219-242.

105. Shin, W.-H., L. Heo, J. Lee, J. Ko, C. Seok, and J. Lee, Ligdockcsa: ProteinLigand Docking Using Conformational Space Annealing. Journal of Computational Chemistry, 2011. 32(15): p. 3226-3232.

106. Chung, J.Y., S.J. Cho, and J.-M. Hah, A Python-Based Docking Program Utilizing a Receptor Bound Ligand Shape: Pythdock. Archives of Pharmacal Research, 2011. 34(9): p. 1451-1458.

107. Plewczynski, D., M. Lazniewski, M. Von Grotthuss, L. Rychlewski, and K. Ginalski, Votedock: Consensus Docking Method for Prediction of Protein-Ligand Interactions. Journal of Computational Chemistry, 2011. 32(4): p. 568-581.

108. Deng, N.-j., P. Zhang, P. Cieplak, and L. Lai, Elucidating the Energetics of Entropically Driven Protein-Ligand Association: Calculations of Absolute Binding Free Energy and Entropy. Journal of Physical Chemistry B, 2011. 115(41): p. 11902-11910.

109. Gallicchio, E., N. Deng, P. He, L. Wickstrom, A.L. Perryman, D.N. Santiago, S. Forli, A.J. Olson, and R.M. Levy, Virtual Screening of Integrase Inhibitors by Large Scale Binding Free Energy Calculations: The Sampl4 Challenge. Journal of Computer-Aided Molecular Design, 2014. 28(4): p. 475-490.

110. Mobley, D.L., S. Liu, N.M. Lim, K.L. Wymer, A.L. Perryman, S. Forli, N. Deng, J. Su, K. Branson, and A.J. Olson, Blind Prediction of Hiv Integrase Binding from the Sampl4 Challenge. Journal of Computer-Aided Molecular Design, 2014. 28(4): p. $327-345$. 
111. Babaoglu, K., A. Simeonov, J.J. Lrwin, M.E. Nelson, B. Feng, C.J. Thomas, L. Cancian, M.P. Costi, D.A. Maltby, A. Jadhav, J. Inglese, C.P. Austin, and B.K. Shoichet, Comprehensive Mechanistic Analysis of Hits from High-Throughput and Docking Screens against Beta-Lactamase. Journal of Medicinal Chemistry, 2008. 51(8): p. 2502-2511.

112. Huang, N. and M.P. Jacobson, Physics-Based Methods for Studying ProteinLigand Interactions. Current Opinion in Drug Discovery \& Development, 2007. 10(3): p. 325-331.

113. Brint, A.T. and P. Willett, Algorithms for the Identification of Three-Dimensional Maximal Common Substructures. Journal of Chemical Information and Computer Sciences, 1987. 27(4): p. 152-158.

114. Fischer, D., R. Norel, H. Wolfson, and R. Nussinov, Surface Motifs by a Computer Vision Technique - Searches, Detection, and Implications for Protein Ligand Recognition. Proteins-Structure Function and Genetics, 1993. 16(3): p. 278-292.

115. Norel, R., D. Fischer, H.J. Wolfson, and R. Nussinov, Molecular-Surface Recognition by a Computer Vision-Based Technique. Protein Engineering, 1994. 7(1): p. 39-46.

116. Miranker, A. and M. Karplus, Functionality Maps of Binding-Sites - a Multiple Copy Simultaneous Search Method. Proteins-Structure Function and Genetics, 1991. 11(1): p. 29-34.

117. Eisen, M.B., D.C. Wiley, M. Karplus, and R.E. Hubbard, Hook - a Program for Finding Novel Molecular Architectures That Satisfy the Chemical and Steric Requirements of a Macromolecule Binding-Site. Proteins-Structure Function and Genetics, 1994. 19(3): p. 199-221. 
118. Bohm, H.J., Ludi - Rule-Based Automatic Design of New Substituents for Enzyme-Inhibitor Leads. Journal of Computer-Aided Molecular Design, 1992. 6(6): p. 593-606.

119. Desjarlais, R.L., R.P. Sheridan, J.S. Dixon, I.D. Kuntz, and R. Venkataraghavan, Docking Flexible Ligands to Macromolecular Receptors by Molecular Shape. Journal of Medicinal Chemistry, 1986. 29(11): p. 2149-2153.

120. Leach, A.R. and I.D. Kuntz, Conformational-Analysis of Flexible Ligands in Macromolecular Receptor-Sites. Journal of Computational Chemistry, 1992. 13(6): p. $730-748$.

121. Goodsell, D.S., H. Lauble, C.D. Stout, and A.J. Olson, Automated Docking in Crystallography - Analysis of the Substrates of Aconitase. Proteins-Structure Function and Genetics, 1993. 17(1): p. 1-10.

122. Oshiro, C.M., I.D. Kuntz, and J.S. Dixon, Flexible Ligand Docking Using a Genetic Algorithm. Journal of Computer-Aided Molecular Design, 1995. 9(2): p. 113-130.

123. Kuntz, I.D., J.M. Blaney, S.J. Oatley, R. Langridge, and T.E. Ferrin, A Geometric Approach to Macromolecule-Ligand Interactions. Journal of Molecular Biology, 1982. 161(2): p. 269-288.

124. Diller, D.J. and K.M. Merz, High Throughput Docking for Library Design and Library Prioritization. Proteins-Structure Function and Genetics, 2001. 43(2): p. 113-124.

125. Schnecke, V. and L.A. Kuhn, Virtual Screening with Solvation and LigandInduced Complementarity. Perspectives in Drug Discovery and Design, 2000. 20(1): p. 171-190. 
126. Zsoldos, Z., D. Reid, A. Simon, B.S. Sadjad, and A.P. Johnson, Ehits: An Innovative Approach to the Docking and Scoring Function Problems. Current Protein \& Peptide Science, 2006. 7(5): p. 421-435.

127. Abagyan, R., M. Totrov, and D. Kuznetsov, Icm - a New Method for Protein Modeling and Design - Applications to Docking and Structure Prediction from the Distorted Native Conformation. Journal of Computational Chemistry, 1994. 15(5): p. 488-506.

128. Accelrys Discovery Studio, Accelrys Software Inc., San Diego, CA, USA, 2012.

129. Verdonk, M.L., J.C. Cole, M.J. Hartshorn, C.W. Murray, and R.D. Taylor, Improved Protein-Ligand Docking Using Gold. Proteins-Structure Function and Genetics, 2003. 52(4): p. 609-623.

130. Clark, K.P. and Ajay, Flexible Ligand Docking without Parameter Adjustment across Four Ligand-Receptor Complexes. Journal of Computational Chemistry, 1995. 16(10): p. 1210-1226.

131. Eldridge, M.D., C.W. Murray, T.R. Auton, G.V. Paolini, and R.P. Mee, Empirical Scoring Functions .1. The Development of a Fast Empirical Scoring Function to Estimate the Binding Affinity of Ligands in Receptor Complexes. Journal of Computer-Aided Molecular Design, 1997. 11(5): p. 425-445.

132. Velec, H.F.G., H. Gohlke, and G. Klebe, Drugscore(Csd)-Knowledge-Based Scoring Function Derived from Small Molecule Crystal Data with Superior Recognition Rate of near-Native Ligand Poses and Better Affinity Prediction. Journal of Medicinal Chemistry, 2005. 48(20): p. 6296-6303.

133. Gohlke, H., M. Hendlich, and G. Klebe, Knowledge-Based Scoring Function to Predict Protein-Ligand Interactions. Journal of Molecular Biology, 2000. 295(2): p. 337-356. 
134. Rognan, D., S.L. Lauemoller, A. Holm, S. Buus, and V. Tschinke, Predicting Binding Affinities of Protein Ligands from Three-Dimensional Models: Application to Peptide Binding to Class I Major Histocompatibility Proteins. Journal of Medicinal Chemistry, 1999. 42(22): p. 4650-4658.

135. Wang, R.X., L.H. Lai, and S.M. Wang, Further Development and Validation of Empirical Scoring Functions for Structure-Based Binding Affinity Prediction. Journal of Computer-Aided Molecular Design, 2002. 16(1): p. 11-26.

136. DeWitte, R.S. and E.I. Shakhnovich, Smog: De Novo Design Method Based on Simple, Fast, and Accurate Free Energy Estimates .1. Methodology and Supporting Evidence. Journal of the American Chemical Society, 1996. 118(47): p. 11733-11744.

137. Kollman, P., Free-Energy Calculations - Applications to Chemical and Biochemical Phenomena. Chemical Reviews, 1993. 93(7): p. 2395-2417.

138. Aqvist, J., V.B. Luzhkov, and B.O. Brandsdal, Ligand Binding Affinities from Md Simulations. Accounts of Chemical Research, 2002. 35(6): p. 358-365.

139. Carlson, H.A. and W.L. Jorgensen, An Extended Linear-Response Method for Determining Free-Energies of Hydration. Journal of Physical Chemistry, 1995. 99(26): p. 10667-10673.

140. Shoichet, B.K., R.M. Stroud, D.V. Santi, I.D. Kuntz, and K.M. Perry, StructureBased Discovery of Inhibitors of Thymidylate Synthase. Science, 1993. 259(5100): p. $1445-1450$.

141. Bohm, H.J., Prediction of Binding Constants of Protein Ligands: A Fast Method for the Prioritization of Hits Obtained from De Novo Design or 3d Database Search Programs. Journal of Computer-Aided Molecular Design, 1998. 12(4): p. 309-323. 
142. Gehlhaar, D.K., G.M. Verkhivker, P.A. Rejto, C.J. Sherman, D.B. Fogel, L.J. Fogel, and S.T. Freer, Molecular Recognition of the Inhibitor Ag-1343 by Hiv-1 Protease - Conformationally Flexible Docking by Evolutionary Programming. Chemistry \& Biology, 1995. 2(5): p. 317-324.

143. Verkhivker, G.M., D. Bouzida, D.K. Gehlhaar, P.A. Rejto, S. Arthurs, A.B. Colson, S.T. Freer, V. Larson, B.A. Luty, T. Marrone, and P.W. Rose, Deciphering Common Failures in Molecular Docking of Ligand-Protein Complexes. Journal of Computer-Aided Molecular Design, 2000. 14(8): p. 731751.

144. Jain, A.N., Scoring Noncovalent Protein-Ligand Interactions: A Continuous Differentiable Function Tuned to Compute Binding Affinities. Journal of Computer-Aided Molecular Design, 1996. 10(5): p. 427-440.

145. Head, R.D., M.L. Smythe, T.I. Oprea, C.L. Waller, S.M. Green, and G.R. Marshall, Validate: A New Method for the Receptor-Based Prediction of Binding Affinities of Novel Ligands. Journal of the American Chemical Society, 1996. 118(16): p. 3959-3969.

146. Gehlhaar, D.K., K.E. Moerder, D. Zichi, C.J. Sherman, R.C. Ogden, and S.T. Freer, De-Novo Design of Enzyme-Inhibitors by Monte-Carlo Ligand Generation. Journal of Medicinal Chemistry, 1995. 38(3): p. 466-472.

147. Muegge, I. and Y.C. Martin, A General and Fast Scoring Function for ProteinLigand Interactions: A Simplified Potential Approach. Journal of Medicinal Chemistry, 1999. 42(5): p. 791-804.

148. Mitchell, J.B.O., R.A. Laskowski, A. Alex, and J.M. Thornton, Bleep - Potential of Mean Force Describing Protein-Ligand Interactions: I. Generating Potential. Journal of Computational Chemistry, 1999. 20(11): p. 1165-1176. 
149. Ishchenko, A.V. and E.I. Shakhnovich, Small Molecule Growth 2001 (Smog2001): An Improved Knowledge-Based Scoring Function for Protein-Ligand Interactions. Journal of Medicinal Chemistry, 2002. 45(13): p. 2770-2780.

150. Feher, M., E. Deretey, and S. Roy, Bhb: A Simple Knowledge-Based Scoring Function to Improve the Efficiency of Database Screening. Journal of Chemical Information and Computer Sciences, 2003. 43(4): p. 1316-1327.

151. Verkhivker, G., K. Appelt, S.T. Freer, and J.E. Villafranca, Empirical Free-Energy Calculations of Ligand-Protein Crystallographic Complexes .1. Knowledge-Based Ligand-Protein Interaction Potentials Applied to the Prediction of HumanImmunodeficiency-Virus-1 Protease Binding-Affinity. Protein Engineering, 1995. 8(7): p. 677-691.

152. Wallqvist, A., R.L. Jernigan, and D.G. Covell, A Preference-Based Free-Energy Parameterization of Enzyme-Inhibitor Binding - Applications to Hiv-1-Protease Inhibitor Design. Protein Science, 1995. 4(9): p. 1881-1903.

153. Corbeil, C.R., P. Englebienne, and N. Moitessier, Docking Ligands into Flexible and Solvated Macromolecules. 1. Development and Validation of Fitted 1.0. Journal of Chemical Information and Modeling, 2007. 47(2): p. 435-449.

154. Leach, A.R., Ligand Docking to Proteins with Discrete Side-Chain Flexibility. Journal of Molecular Biology, 1994. 235(1): p. 345-356.

155. Sherman, W., T. Day, M.P. Jacobson, R.A. Friesner, and R. Farid, Novel Procedure for Modeling Ligand/Receptor Induced Fit Effects. Journal of Medicinal Chemistry, 2006. 49(2): p. 534-553.

156. Meiler, J. and D. Baker, Rosettaligand: Protein-Small Molecule Docking with Full Side-Chain Flexibility. Proteins-Structure Function and Bioinformatics, 2006. 65(3): p. 538-548. 
157. Cozzini, P., G.E. Kellogg, F. Spyrakis, D.J. Abraham, G. Costantino, A. Emerson, F. Fanelli, H. Gohlke, L.A. Kuhn, G.M. Morris, M. Orozco, T.A. Pertinhez, M. Rizzi, and C.A. Sotriffer, Target Flexibility: An Emerging Consideration in Drug Discovery and Design. Journal of Medicinal Chemistry, 2008. 51(20): p. 62376255.

158. Wei, B.Q., L.H. Weaver, A.M. Ferrari, B.W. Matthews, and B.K. Shoichet, Testing a Flexible-Receptor Docking Algorithm in a Model Binding Site. Journal of Molecular Biology, 2004. 337(5): p. 1161-1182.

159. Mobley, D.L. and K.A. Dill, Binding of Small-Molecule Ligands to Proteins: "What You See" Is Not Always "What You Get". Structure, 2009. 17(4): p. 489498.

160. Mobley, D.L., J.D. Chodera, and K.A. Dill, Confine-and-Release Method: Obtaining Correct Binding Free Energies in the Presence of Protein Conformational Change. Journal of Chemical Theory and Computation, 2007. 3(4): p. 1231-1235.

161. Mobley, D.L., A.P. Graves, J.D. Chodera, A.C. McReynolds, B.K. Shoichet, and K.A. Dill, Predicting Absolute Ligand Binding Free Energies to a Simple Model Site. Journal of Molecular Biology, 2007. 371(4): p. 1118-1134.

162. Najmanovich, R., J. Kuttner, V. Sobolev, and M. Edelman, Side-Chain Flexibility in Proteins Upon Ligand Binding. Proteins-Structure Function and Genetics, 2000. 39(3): p. 261-268.

163. Chou, J.J. and A. Bax, Protein Side-Chain Rotamers from Dipolar Couplings in a Liquid Crystalline Phase. Journal of the American Chemical Society, 2001. 123(16): p. 3844-3845. 
164. Morton, A. and B.W. Matthews, Specificity of Ligand-Binding in a Buried Nonpolar Cavity of T4 Lysozyme - Linkage of Dynamics and Structural Plasticity. Biochemistry, 1995. 34(27): p. 8576-8588.

165. Zhang, X.J., J.A. Wozniak, and B.W. Matthews, Protein Flexibility and Adaptability Seen in 25 Crystal Forms of T4 Lysozyme. Journal of Molecular Biology, 1995. 250(4): p. 527-552.

166. Ma, B.Y., M. Shatsky, H.J. Wolfson, and R. Nussinov, Multiple Diverse Ligands Binding at a Single Protein Site: A Matter of Pre-Existing Populations. Protein Science, 2002. 11(2): p. 184-197.

167. Eisenmesser, E.Z., D.A. Bosco, M. Akke, and D. Kern, Enzyme Dynamics During Catalysis. Science, 2002. 295(5559): p. 1520-1523.

168. Fragai, M., C. Luchinat, and G. Parigi, "Four-Dimensional" Protein Structures: Examples from Metalloproteins. Accounts of Chemical Research, 2006. 39(12): p. 909-917.

169. Gerstein, M., A.M. Lesk, and C. Chothia, Structural Mechanisms for Domain Movements in Proteins. Biochemistry, 1994. 33(22): p. 6739-6749.

170. Beier, C. and M. Zacharias, Tackling the Challenges Posed by Target Flexibility in Drug Design. Expert Opinion on Drug Discovery, 2010. 5(4): p. 347-359.

171. Carlson, H.A., K.M. Masukawa, and J.A. McCammon, Method for Including the Dynamic Fluctuations of a Protein in Computer-Aided Drug Design. Journal of Physical Chemistry A, 1999. 103(49): p. 10213-10219.

172. Kokh, D.B., R.C. Wade, and W. Wenzel, Receptor Flexibility in Small-Molecule Docking Calculations. Wiley Interdisciplinary Reviews-Computational Molecular Science, 2011. 1(2): p. 298-314. 
173. Fernandez-Recio, J., M. Totrov, and R. Abagyan, Soft Protein-Protein Docking in Internal Coordinates. Protein Science, 2002. 11(2): p. 280-291.

174. Vieth, M., J.D. Hirst, A. Kolinski, and C.L. Brooks, Assessing Energy Functions for Flexible Docking. Journal of Computational Chemistry, 1998. 19(14): p. 16121622.

175. Wu, G.S., D.H. Robertson, C.L. Brooks, and M. Vieth, Detailed Analysis of GridBased Molecular Docking: A Case Study of Cdocker - a Charmm-Based Md Docking Algorithm. Journal of Computational Chemistry, 2003. 24(13): p. 15491562.

176. Erickson, J.A., M. Jalaie, D.H. Robertson, R.A. Lewis, and M. Vieth, Lessons in Molecular Recognition: The Effects of Ligand and Protein Flexibility on Molecular Docking Accuracy. Journal of Medicinal Chemistry, 2004. 47(1): p. 4555.

177. Ferrari, A.M., B.Q.Q. Wei, L. Costantino, and B.K. Shoichet, Soft Docking and Multiple Receptor Conformations in Virtual Screening. Journal of Medicinal Chemistry, 2004. 47(21): p. 5076-5084.

178. Dunbrack, R.L. and M. Karplus, Backbone-Dependent Rotamer Library for Proteins - Application to Side-Chain Prediction. Journal of Molecular Biology, 1993. 230(2): p. 543-574.

179. Tuffery, P., C. Etchebest, S. Hazout, and R. Lavery, A New Approach to the Rapid-Determination of Protein Side-Chain Conformations. Journal of Biomolecular Structure \& Dynamics, 1991. 8(6): p. 1267-1289.

180. Lovell, S.C., J.M. Word, J.S. Richardson, and D.C. Richardson, The Penultimate Rotamer Library. Proteins-Structure Function and Genetics, 2000. 40(3): p. 389408. 
181. Schaffer, L. and G.M. Verkhivker, Predicting Structural Effects in Hiv-1 Protease Mutant Complexes with Flexible Ligand Docking and Protein Side-Chain Optimization. Proteins-Structure Function and Genetics, 1998. 33(2): p. 295-310.

182. Carlson, H.A. and J.A. McCammon, Accommodating Protein Flexibility in Computational Drug Design. Molecular Pharmacology, 2000. 57(2): p. 213-218.

183. Huang, S.Y. and X.Q. Zou, Ensemble Docking of Multiple Protein Structures: Considering Protein Structural Variations in Molecular Docking. ProteinsStructure Function and Bioinformatics, 2007. 66(2): p. 399-421.

184. Carlson, H.A., K.M. Masukawa, K. Rubins, F.D. Bushman, W.L. Jorgensen, R.D. Lins, J.M. Briggs, and J.A. McCammon, Developing a Dynamic Pharmacophore Model for Hiv-1 Integrase. Journal of Medicinal Chemistry, 2000. 43(11): p. 2100-2114.

185. Totrov, M. and R. Abagyan, Flexible Ligand Docking to Multiple Receptor Conformations: A Practical Alternative. Current Opinion in Structural Biology, 2008. 18(2): p. 178-184.

186. Barril, X. and S.D. Morley, Unveiling the Full Potential of Flexible Receptor Docking Using Multiple Crystallographic Structures. Journal of Medicinal Chemistry, 2005. 48(13): p. 4432-4443.

187. Allen, M.P., Introduction to Molecular Dynamics Simulation, In: Computational Soft Matter-from Synthetic Polymers to Proteins. NIC Series, 2004. 23: p. 1-28.

188. Warshel, A., Molecular Dynamics Simulations of Biological Reactions. Accounts of Chemical Research, 2002. 35(6): p. 385-395.

189. Rapaport, D.C., The Art of Molecular Dynamics Simulation. 2004: Cambridge University Press. 
190. Broughton, H.B., A Method for Including Protein Flexibility in Protein-Ligand Docking: Improving Tools for Database Mining and Virtual Screening. Journal of Molecular Graphics \& Modelling, 2000. 18(3): p. 247-+.

191. Zacharias, M., Rapid Protein-Ligand Docking Using Soft Modes from Molecular Dynamics Simulations to Account for Protein Deformability: Binding of Fk506 to Fkbp. Proteins-Structure Function and Bioinformatics, 2004. 54(4): p. 759-767.

192. Brunne, R.M., K.D. Berndt, P. Guntert, K. Wuthrich, and W.F. Vangunsteren, Structure and Internal Dynamics of the Bovine Pancreatic Trypsin-Inhibitor in Aqueous-Solution from Long-Time Molecular-Dynamics Simulations. ProteinsStructure Function and Genetics, 1995. 23(1): p. 49-62.

193. Eyrisch, S. and V. Helms, Transient Pockets on Protein Surfaces Involved in Protein-Protein Interaction. Journal of Medicinal Chemistry, 2007. 50(15): p. 3457-3464.

194. Philippopoulos, M. and C. Lim, Exploring the Dynamic Information Content of a Protein Nmr Structure: Comparison of a Molecular Dynamics Simulation with the Nmr and X-Ray Structures of Escherichia Coli Ribonuclease Hi. ProteinsStructure Function and Genetics, 1999. 36(1): p. 87-110.

195. da Silva, M.L., A. da Silva Gonçalves, P.R. Batista, J.D. Figueroa-Villar, P.G. Pascutti, and T.C.C. França, Design, Docking Studies and Molecular Dynamics of New Potential Selective Inhibitors of Plasmodium Falciparum Serine Hydroxymethyltransferase. Molecular Simulation, 2010. 36(1): p. 5-14.

196. Yang, Z., Y. Nie, G. Yang, Y. Zu, Y. Fu, and L. Zhou, Synergistic Effects in the Designs of Neuraminidase Ligands: Analysis from Docking and Molecular Dynamics Studies. Journal of Theoretical Biology, 2010. 267: p. 363-374.

197. Wang, X., W. Yang, X. Xu, H. Zhang, Y. Li, and Y. Wang, Studies of Benzothiadiazine Derivatives as Hepatitis C Virus Ns5b Polymerase Inhibitors 
Using 3d-Qsar, Molecular Docking and Molecular Dynamics. Current Medicinal Chemistry, 2010. 17(25): p. 2788-2803.

198. Mandlik, V. and S. Singh, Molecular Docking and Molecular Dynamics Simulation Study of Inositol Phosphorylceramide Synthase ? Inhibitor Complex in Leishmaniasis: Insight into the Structure Based Drug Design [Version 2; Referees: 2 Approved]. Vol. 5. 2016.

199. Alonso, H., A.A. Bliznyuk, and J.E. Gready, Combining Docking and Molecular Dynamic Simulations in Drug Design. Medicinal Research Reviews, 2006. 26(5): p. 531-568.

200. Schlitter, J., M. Engels, and P. Krüger, Targeted Molecular Dynamics: A New Approach for Searching Pathways of Conformational Transitions. Journal of Molecular Graphics, 1994. 12(2): p. 84-89.

201. Samsonov, S.A., J.-P. Gehrcke, and M.T. Pisabarro, Flexibility and Explicit Solvent in Molecular-Dynamics-Based Docking of Protein-Glycosaminoglycan Systems. Journal of Chemical Information and Modeling, 2014. 54(2): p. 582-592.

202. Bleicher, K.H., H.J. Bohm, K. Muller, and A.I. Alanine, Hit and Lead Generation: Beyond High-Throughput Screening. Nature Reviews Drug Discovery, 2003. 2(5): p. $369-378$.

203. Keseru, G.M. and G.M. Makara, Hit Discovery and Hit-to-Lead Approaches. Drug Discovery Today, 2006. 11(15-16): p. 741-748.

204. Krovat, E.M., K.H. Fruhwirth, and T. Langer, Pharmacophore Identification, in Silico Screening, and Virtual Library Design for Inhibitors of the Human Factor Xa. Journal of Chemical Information and Modeling, 2005. 45(1): p. 146-159.

205. Güner, O.F., Pharmacophore Perception, Development, and Use in Drug Design. Iul Biotechnology Series. 2000: LaJolla, CA : International University Line, c2000. 
206. Langer, T. and R.D. Hoffmann, Pharmacophores and Pharmacophore Searches. Methods and Principles in Medicinal Chemistry: 32. 2006: Weinheim : WileyVCH ; [Chichester : John Wiley, distributor], c2006.

207. Testa, B. and L.B. Kier, The Concept of Molecular-Structure in Structure Activity Relationship Studies and Drug Design. Medicinal Research Reviews, 1991. 11(1): p. 35-48.

208. Wang, T., M.B. Wu, J.P. Lin, and L.R. Yang, Quantitative Structure-Activity Relationship: Promising Advances in Drug Discovery Platforms. Expert Opinion on Drug Discovery, 2015. 10(12): p. 1283-1300.

209. Patel, H.M., M.N. Noolvi, P. Sharma, V. Jaiswal, S. Bansal, S. Lohan, S.S. Kumar, V. Abbot, S. Dhiman, and V. Bhardwaj, Quantitative Structure-Activity Relationship (Qsar) Studies as Strategic Approach in Drug Discovery. Medicinal Chemistry Research, 2014. 23(12): p. 4991-5007.

210. Ye, Q., R.M. Krug, and Y.J. Tao, The Mechanism by Which Influenza a Virus Nucleoprotein Forms Oligomers and Binds RNA. Nature, 2006. 444(7122): p. 1078-1082.

211. Shu, L.L., W.J. Bean, and R.G. Webster, Analysis of the Evolution and Variation of the Human Influenza-a Virus Nucleoprotein Gene from 1933 to 1990. Journal of Virology, 1993. 67(5): p. 2723-2729.

212. Li, Z., T. Watanabe, M. Hatta, S. Watanabe, A. Nanbo, M. Ozawa, S. Kakugawa, M. Shimojima, S. Yamada, G. Neumann, and Y. Kawaoka, Mutational Analysis of Conserved Amino Acids in the Influenza a Virus Nucleoprotein. Journal of Virology, 2009. 83(9): p. 4153-4162.

213. Ng, A.K.-L., J.-H. Wang, and P.-C. Shaw, Structure and Sequence Analysis of Influenza a Virus Nucleoprotein. Science in China Series C-Life Sciences, 2009. 52(5): p. 439-449. 
214. Shen, Y.-F., Y.-H. Chen, S.-Y. Chu, M.-I. Lin, H.-T. Hsu, P.-Y. Wu, C.-J. Wu, H.W. Liu, F.-Y. Lin, G. Lin, P.-H. Hsu, A.-S. Yang, Y.-S.E. Cheng, Y.-T. Wu, C.-H. Wong, and M.-D. Tsai, E339 ... R416 Salt Bridge of Nucleoprotein as a Feasible Target for Influenza Virus Inhibitors. Proceedings of the National Academy of Sciences of the United States, 2011(40): p. 16515.

215. Fedichev, P., R. Timakhov, T. Pyrkov, E. Getmantsev, and A. Vinnik, StructureBased Drug Design of a New Chemical Class of Small Molecules Active against Influenza a Nucleoprotein in Vitro and in Vivo. PLoS Currents, 2011. 3: p. RRN1253.

216. Kao, R.Y., D. Yang, L.S. Lau, W.H.W. Tsui, L.H. Hu, J. Dai, M.P. Chan, C.M. Chan, P. Wang, B.J. Zheng, J.A. Sun, J.D. Huang, J. Madar, G.H. Chen, H.L. Chen, Y. Guan, and K.Y. Yuen, Identification of Influenza a Nucleoprotein as an Antiviral Target. Nature Biotechnology, 2010. 28(6): p. 600-U88.

217. Lejal, N., B. Tarus, E. Bouguyon, S. Chenavas, N. Bertho, B. Delmas, R.W.H. Ruigrok, C. Di Primo, and A. Slama-Schwok, Structure-Based Discovery of the Novel Antiviral Properties of Naproxen against the Nucleoprotein of Influenza a Virus. Antimicrobial Agents and Chemotherapy, 2013. 57(5): p. 2231-2242.

218. Cheng, H., J. Wan, M.-I. Lin, Y. Liu, X. Lu, J. Liu, Y. Xu, J. Chen, Z. Tu, Y.-S.E. Cheng, and K. Ding, Design, Synthesis, and in Vitro Biological Evaluation of 1h1,2,3-Triazole-4-Carboxamide Derivatives as New Anti-Influenza a Agents Targeting Virus Nucleoprotein. Journal of Medicinal Chemistry, 2012. 55(5): p. 2144-2153.

219. Su, C.-Y., T.-J.R. Cheng, M.-I. Lin, S.-Y. Wang, W.-I. Huang, S.-Y. Lin-Chu, Y.H. Chen, C.-Y. Wu, M.M.C. Lai, W.-C. Cheng, Y.-T. Wu, M.-D. Tsai, Y.-S.E. Cheng, and C.-H. Wong, High-Throughput Identification of Compounds Targeting Influenza RNA-Dependent RNA Polymerase Activity. Proceedings of the National 
Academy of Sciences of the United States of America, 2010. 107(45): p. 1915119156.

220. Gerritz, S.W., C. Cianci, S. Kim, B.C. Pearce, C. Deminie, L. Discotto, B. McAuliffe, B.F. Minassian, S. Shi, S. Zhu, W. Zhai, A. Pendri, G. Li, M.A. Poss, S. Edavettal, P.A. McDonnell, H.A. Lewis, K. Maskos, M. Moertl, R. Kiefersauer, S. Steinbacher, E.T. Baldwin, W. Metzler, J. Bryson, M.D. Healy, T. Philip, M. Zoeckler, R. Schartman, M. Sinz, V.H. Leyva-Grado, H.-H. Hoffmann, D.R. Langley, N.A. Meanwell, and M. Krystal, Inhibition of Influenza Virus Replication Via Small Molecules That Induce the Formation of Higher-Order Nucleoprotein Oligomers. Proceedings of the National Academy of Sciences of the United States of America, 2011. 108(37): p. 15366-15371.

221. Jones, L.J.F., R. Carballido-Lopez, and J. Errington, Control of Cell Shape in Bacteria: Helical, Actin-Like Filaments in Bacillus Subtilis. Cell, 2001. 104(6): p. 913-922.

222. Varley, A.W. and G.C. Stewart, The Divivb Region of the Bacillus-Subtilis Chromosome Encodes Homologs of Escherichia-Coli Septum Placement (Mincd) and Cell-Shape (Mrebcd) Determinants. Journal of Bacteriology, 1992. 174(21): p. 6729-6742.

223. Kruse, T., J. Bork-Jensen, and K. Gerdes, The Morphogenetic MreBCD Proteins of Escherichia Coli Form an Essential Membrane-Bound Complex. Molecular Microbiology, 2005. 55(1): p. 78-89.

224. Figge, R.M., A.V. Divakaruni, and J.W. Gober, MreB, the Cell ShapeDetermining Bacterial Actin Homologue, Co-Ordinates Cell Wall Morphogenesis in Caulobacter Crescentus. Molecular Microbiology, 2004. 51(5): p. 1321-1332.

225. Bean, G.J. and K.J. Amann, Polymerization Properties of the Thermotoga Maritima Actin MreB: Roles of Temperature, Nucleotides, and Ions. Biochemistry, 2008. 47(2): p. 826-835. 
226. Slovak, P.M., G.H. Wadhams, and J.P. Armitage, Localization of MreB in Rhodobacter Sphaeroides under Conditions Causing Changes in Cell Shape and Membrane Structure. Journal of Bacteriology, 2005. 187(1): p. 54-64.

227. Costa, C.S. and D.N. Anton, Conditional Lethality of Cell Shape Mutations of Salmonella Typhimurium: Roda and Mre Mutants Are Lethal on Solid but Not in Liquid Medium. Current Microbiology, 1999. 38(3): p. 137-142.

228. Costa, C.S. and D.N. Anton, Round-Cell Mutants of Salmonella-Typhimurium Produced by Transposition Mutagenesis - Lethality of Roda and Mre Mutations. Molecular and General Genetics, 1993. 236(2-3): p. 387-394.

229. Burger, A., K. Sichler, G. Kelemen, M. Buttner, and W. Wohlleben, Identification and Characterization of the Mre Gene Region of Streptomyces Coelicolor A3(2). Molecular and General Genetics, 2000. 263(6): p. 1053-1060.

230. Mazza, P., E.E. Noens, K. Schirner, N. Grantcharova, A.M. Mommaas, H.K. Koerten, G. Muth, K. Flardh, G.P. Van Wezel, and W. Wohlleben, MreB of Streptomyces Coelicolor Is Not Essential for Vegetative Growth but Is Required for the Integrity of Aerial Hyphae and Spores. Molecular Microbiology, 2006. 60(4): p. 838-852.

231. Robertson, G.T., T.B. Doyle, Q. Du, L. Duncan, K.E. Mdluli, and A.S. Lynch, A Novel Indole Compound That Inhibits Pseudomonas Aeruginosa Growth by Targeting MreB Is a Substrate for Mexab-Oprm. Journal of Bacteriology, 2007. 189(19): p. 6870-6881.

232. Doi, M., M. Wachi, F. Ishino, S. Tomioka, M. Ito, Y. Sakagami, A. Suzuki, and M. Matsuhashi, Determinations of the DNA-Sequence of the MreB-Gene and of the Gene-Products of the Mre-Region That Function in Formation of the Rod Shape of Escherichia Coli Cells. Journal of Bacteriology, 1988. 170(10): p. 4619-4624. 
233. van den Ent, F., L.A. Amos, and J. Lowe, Prokaryotic Origin of the Actin Cytoskeleton. Nature, 2001. 413(6851): p. 39-44.

234. Esue, O., M. Cordero, D. Wirtz, and Y. Tseng, The Assembly of MreB, a Prokaryotic Homolog of Actin. Journal of Biological Chemistry, 2005. 280(4): p. 2628-2635.

235. Esue, O., D. Wirtz, and Y. Tseng, GTPase Activity, Structure, and Mechanical Properties of Filaments Assembled from Bacterial Cytoskeleton Protein MreB. Journal of Bacteriology, 2006. 188(3): p. 968-976.

236. Srinivasan, R., M. Mishra, M. Murata-Hori, and M.K. Balasubramanian, Filament Formation of the Escherichia Coli Actin-Related Protein, MreB, in Fission Yeast. Current Biology, 2007. 17(3): p. 266-272.

237. Wachi, M., M. Doi, S. Tamaki, W. Park, S. Nakajimaiijima, and M. Matsuhashi, Mutant Isolation and Molecular-Cloning of Mre-Genes, Which Determine CellShape, Sensitivity to Mecillinam, and Amount of Penicillin-Binding Proteins in Escherichia-Coli. Journal of Bacteriology, 1987. 169(11): p. 4935-4940.

238. van den Ent, F., T. Izore, T.A.M. Bharat, C.M. Johnson, and J. Loewe, Bacterial Actin MreB Forms Antiparallel Double Filaments. Elife, 2014. 3: p. 1-22.

239. Wachi, M. and M. Matsuhashi, Negative Control of Cell-Division by MreB, a Gene That Functions in Determining the Rod Shape of Escherichia Coli Cells. Journal of Bacteriology, 1989. 171(6): p. 3123-3127.

240. Soufo, H.J.D. and P.L. Graumann, Actin-Like Proteins MreB and Mbl from Bacillus Subtilis Are Required for Bipolar Positioning of Replication Origins. Current Biology, 2003. 13(21): p. 1916-1920.

241. Kruse, T. and K. Gerdes, Bacterial DNA Segregation by the Actin-Like MreB Protein. Trends in Cell Biology, 2005. 15(7): p. 343-345. 
242. Kruse, T., J. Moller-Jensen, A. Lobner-Olesen, and K. Gerdes, Dysfunctional MreB Inhibits Chromosome Segregation in Escherichia Coli. Embo Journal, 2003. 22(19): p. 5283-5292.

243. Gital, Z., N.A. Dye, A. Reisenauer, M. Wachi, and L. Shapiro, MreB ActinMediated Segregation of a Specific Region of a Bacterial Chromosome. Cell, 2005. 120(3): p. 329-341.

244. Soufo, H.J.D. and P.L. Graumann, Bacillus Subtilis Actin-Like Protein MreB Influences the Positioning of the Replication Machinery and Requires Membrane Proteins MreC/D and Other Actin-Like Proteins for Proper Localization. Bmc Cell Biology, 2005. 6: p. 1-11.

245. Gitai, Z., N. Dye, and L. Shapiro, An Actin-Like Gene Can Determine Cell Polarity in Bacteria. Proceedings of the National Academy of Sciences of the United States of America, 2004. 101(23): p. 8643-8648.

246. Popp, D., A. Narita, K. Maeda, T. Fujisawa, U. Ghoshdastider, M. Iwasa, Y. Maeda, and R.C. Robinson, Filament Structure, Organization, and Dynamics in MreB Sheets. Journal of Biological Chemistry, 2010. 285(21): p. 15858-15865.

247. Gunning, P.W., U. Ghoshdastider, S. Whitaker, D. Popp, and R.C. Robinson, The Evolution of Compositionally and Functionally Distinct Actin Filaments. Journal of Cell Science, 2015. 128(11): p. 2009-2019.

248. White, C.L. and J.W. Gober, MreB: Pilot or Passenger of Cell Wall Synthesis? Trends in Microbiology, 2012. 20(2): p. 74-79.

249. Vollmer, W., The Prokaryotic Cytoskeleton: A Putative Target for Inhibitors and Antibiotics? Applied Microbiology and Biotechnology, 2006. 73(1): p. 37-47.

250. Iwai, N., K. Nagai, and M. Wachi, Novel S-Benzylisothiourea Compound That Induces Spherical Cells in Escherichia Coli Probably by Acting on a Rod-Shape- 
Determining Protein $(S)$ Other Than Penicillin-Binding Protein 2. Bioscience, Biotechnology, and Biochemistry, 2002. 66(12): p. 2658-2662.

251. Iwai, N., T. Ebata, H. Nagura, T. Kitazume, K. Nagai, and M. Wachi, StructureActivity Relationship of S-Benzylisothiourea Derivatives to Induce Spherical Cells in Escherichia Coli. Bioscience Biotechnology and Biochemistry, 2004. 68(11): p. 2265-2269.

252. Iwai, N., T. Fujii, H. Nagura, M. Wachi, and T. Kitazume, Structure-Activity Relationship Study of the Bacterial Actin-Like Protein MreB Inhibitors: Effects' of Substitution of Benzyl Group in S-Benzyliothiourea. Bioscience Biotechnology and Biochemistry, 2007. 71(1): p. 246-248.

253. Bean, G.J., S.T. Fllckinger, W.M. Westler, M.E. McCully, D. Sept, D.B. Weibel, and K.J. Amann, A22 Disrupts the Bacterial Actin Cytoskeleton by Directly Binding and Inducing a Low-Affinity State in MreB. Biochemistry, 2009. 48(22): p. $4852-4857$.

254. Fradera, X., X. de la Cruz, C. Silva, J.L. Gelpi, F.J. Luque, and M. Orozco, Ligand-Induced Changes in the Binding Sites of Proteins. Bioinformatics, 2002. 18(7): p. 939-948.

255. Murray, C.W., C.A. Baxter, and A.D. Frenkel, The Sensitivity of the Results of Molecular Docking to Induced Fit Effects: Application to Thrombin, Thermolysin and Neuraminidase. Journal of Computer-Aided Molecular Design, 1999. 13(6): p. $547-562$.

256. Todd, A.E., C.A. Orengo, and J.M. Thornton, Plasticity of Enzyme Active Sites. Trends in Biochemical Sciences, 2002. 27(8): p. 419-426.

257. Kokkinidis, M., N.M. Glykos, and V.E. Fadouloglou, Protein Flexibility and Enzymatic Catalysis, in Structural and Mechanistic Enzymology: Bringing 
Together Experiments and Computing, C. Christov and T. KarabenchevaChristova, Editors. 2012. p. 181-218.

258. Arnold, K., L. Bordoli, J. Kopp, and T. Schwede, The Swiss-Model Workspace: A Web-Based Environment for Protein Structure Homology Modelling. Bioinformatics, 2006. 22(2): p. 195-201.

259. Hess, B., Gromacs 4: Algorithms for Highly Efficient, Load-Balanced, and Scalable Molecular Simulation. Abstracts of Papers of the American Chemical Society, 2009. 237.

260. Cornell, W.D., P. Cieplak, C.I. Bayly, I.R. Gould, K.M. Merz, Jr., D.M. Ferguson, D.C. Spellmeyer, T. Fox, J.W. Caldwell, and P.A. Kollman, A Second Generation Force Field for the Simulation of Proteins, Nucleic Acids, and Organic Molecules. Journal of the American Chemical Society, 1995(19): p. 5179-5197.

261. Bussi, G., D. Donadio, and M. Parrinello, Canonical Sampling through Velocity Rescaling. Journal of Chemical Physics, 2007. 126(1): p. 1-7.

262. Pettersen, E.F., T.D. Goddard, C.C. Huang, G.S. Couch, D.M. Greenblatt, E.C. Meng, and T.E. Ferrin, UCSF Chimera - a Visualization System for Exploratory Research and Analysis. Journal of Computational Chemistry, 2004. 25(13): p. 1605-1612.

263. Jorgensen, W.L., J. Chandrasekhar, J.D. Madura, R.W. Impey, and M.L. Klein, Comparison of Simple Potential Functions for Simulating Liquid Water. Journal of Chemical Physics, 1983. 79(2): p. 926-935.

264. Darden, T., D. York, and L. Pedersen, Particle Mesh Ewald - an N.Log(N) Method for Ewald Sums in Large Systems. Journal of Chemical Physics, 1993. 98(12): p. 10089-10092. 
265. Hess, B., C. Kutzner, D. van der Spoel, and E. Lindahl, Gromacs 4: Algorithms for Highly Efficient, Load-Balanced, and Scalable Molecular Simulation. Journal of Chemical Theory and Computation, 2008. 4(3): p. 435-447.

266. Frisch, M.J., G.W. Trucks, H.B. Schlegel, G.E. Scuseria, M.A. Robb, J.R. Cheeseman, G. Scalmani, V. Barone, B. Mennucci, G.A. Petersson, H. Nakatsuji, M. Caricato, X. Li, H.P. Hratchian, A.F. Izmaylov, J. Bloino, G. Zheng, J.L. Sonnenberg, M. Hada, M. Ehara, K. Toyota, R. Fukuda, J. Hasegawa, M. Ishida, T. Nakajima, Y. Honda, O. Kitao, H. Nakai, T. Vreven, J.A. Montgomery Jr., J.E. Peralta, F. Ogliaro, M.J. Bearpark, J. Heyd, E.N. Brothers, K.N. Kudin, V.N. Staroverov, R. Kobayashi, J. Normand, K. Raghavachari, A.P. Rendell, J.C. Burant, S.S. Iyengar, J. Tomasi, M. Cossi, N. Rega, N.J. Millam, M. Klene, J.E. Knox, J.B. Cross, V. Bakken, C. Adamo, J. Jaramillo, R. Gomperts, R.E. Stratmann, O. Yazyev, A.J. Austin, R. Cammi, C. Pomelli, J.W. Ochterski, R.L. Martin, K. Morokuma, V.G. Zakrzewski, G.A. Voth, P. Salvador, J.J. Dannenberg, S. Dapprich, A.D. Daniels, Ö. Farkas, J.B. Foresman, J.V. Ortiz, J. Cioslowski, and D.J. Fox, Gaussian 09. 2009, Gaussian, Inc.: Wallingford, CT, USA.

267. Dupradeau, F.-Y., A. Pigache, T. Zaffran, C. Savineau, R. Lelong, N. Grivel, D. Lelong, W. Rosanski, and P. Cieplak, The R.E.D. Tools: Advances in RESP and ESP Charge Derivation and Force Field Library Building. Physical Chemistry Chemical Physics, 2010. 12(28): p. 7821-7839.

268. Wang, J., W. Wang, P.A. Kollman, and D.A. Case, Automatic Atom Type and Bond Type Perception in Molecular Mechanical Calculations. Journal of Molecular Graphics \& Modelling, 2006. 25(2): p. 247-260.

269. Case, D.A., T.E. Cheatham, T. Darden, H. Gohlke, R. Luo, K.M. Merz, A. Onufriev, C. Simmerling, B. Wang, and R.J. Woods, The Amber Biomolecular Simulation Programs. Journal of Computational Chemistry, 2005. 26(16): p. 16681688. 
270. Gold User Manual. 10/06/2016]; Available from: http://www.ccdc.cam.ac.uk/Lists/DocumentationList.

271. Rastelli, G., Emerging Topics in Structure-Based Virtual Screening. Pharmaceutical Research, 2013(5): p. 1458.

272. Sink, R., S. Gobec, S. Pecar, and A. Zega, False Positives in the Early Stages of Drug Discovery. Current Medicinal Chemistry, 2010. 17(34): p. 4231-4255.

273. Jones, L.J., R. Carballido-Lopez, and J. Errington, Control of Cell Shape in Bacteria: Helical, Actin-Like Filaments in Bacillus Subtilis. Cell, 2001. 104.

274. Formstone, A. and J. Errington, A Magnesium-Dependent Mreb Null Mutant: Implications for the Role of Mreb in Bacillus Subtilis. Molecular Microbiology, 2005. 55(6): p. 1646-1657.

275. Cabeen, M.T. and C. Jacobs-Wagner, The Bacterial Cytoskeleton, in Annual Review of Genetics, Vol 44, A. Campbell, M. Lichten, and G. Schupbach, Editors. 2010. p. 365-392.

276. Karczmarek, A., R.M.-A. Baselga, S. Alexeeva, F.G. Hansen, M. Vicente, N. Nanninga, and T. den Blaauwen, DNA and Origin Region Segregation Are Not Affected by the Transition from Rod to Sphere after Inhibition of Escherichia Coli MreB by A22. Molecular Microbiology, 2007. 65(1): p. 51-63.

277. Takacs, C.N., S. Poggio, G. Charbon, M. Pucheault, W. Vollmer, and C. JacobsWagner, MreB Drives De Novo Rod Morphogenesis in Caulobacter Crescentus Via Remodeling of the Cell Wall. Journal of Bacteriology, 2010. 192(6): p. 16711684.

278. Hernando-Amado, S., P. Blanco, M. Alcalde-Rico, F. Corona, J.A. RealesCalderón, M.B. Sánchez, and J.L. Martínez, Multidrug Efflux Pumps as Main Players in Intrinsic and Acquired Resistance to Antimicrobials. Drug Resistance Updates, 2016. 28: p. 13-27. 
279. Gillis, N.K. and H.L. McLeod, The Pharmacogenomics of Drug Resistance to Protein Kinase Inhibitors. Drug Resistance Updates, 2016. 28: p. 28-42.

280. Andersson, D.I., D. Hughes, and J.Z. Kubicek-Sutherland, Mechanisms and Consequences of Bacterial Resistance to Antimicrobial Peptides. Drug Resistance Updates, 2016. 26: p. 43-57.

281. Ventola, C.L., The Antibiotic Resistance Crisis: Part 1: Causes and Threats. Pharmacy and Therapeutics, 2015. 40(4): p. 277-283.

282. Awuni, Y. and Y. Mu, Reduction of False Positives in Structure-Based Virtual Screening When Receptor Plasticity Is Considered. Molecules, 2015. 20(3): p. 5152-5164.

283. Wallace, A.C., R.A. Laskowski, and J.M. Thornton, Ligplot - a Program to Generate Schematic Diagrams of Protein Ligand Interactions. Protein Engineering, 1995. 8(2): p. 127-134.

284. Kruse, T., B. Blagoev, A. Lobner-Olesen, M. Wachi, K. Sasaki, N. Iwai, M. Mann, and K. Gerdes, Actin Homolog MreB and RNA Polymerase Interact and Are Both Required for Chromosome Segregation in Escherichia Coli. Genes \& Development, 2006. 20(1): p. 113-124.

285. Paissoni, C., D. Spiliotopoulos, G. Musco, and A. Spitaleri, GMXPBSA 2.0: A Gromacs Tool to Perform MM/PBSA and Computational Alanine Scanning. Computer Physics Communications, 2014. 185(11): p. 2920-2929.

286. Matte, A., L.W. Tari, and L.T.J. Delbaere, How Do Kinases Transfer Phosphoryl Groups? Structure with Folding \& Design, 1998. 6(4): p. 413-419.

287. Harrison, C.B. and K. Schulten, Quantum and Classical Dynamics Simulations of ATP Hydrolysis in Solution. Journal of Chemical Theory and Computation, 2012. 8(7): p. 2328-2335. 
288. Parke, C.L., E.J. Wojcik, S. Kim, and D.K. Worthylake, ATP Hydrolysis in Eg5 Kinesin Involves a Catalytic Two-Water Mechanism. Journal of Biological Chemistry, 2010. 285(8): p. 5859-5867.

289. Grigorenko, B.L., A.V. Nemukhin, M.S. Shadrina, I.A. Topol, and S.K. Burt, Mechanisms of Guanosine Triphosphate Hydrolysis by Ras and Ras-GAP Proteins as Rationalized by $\mathrm{Ab}$ Initio QM/MM Simulations. Proteins-Structure Function and Bioinformatics, 2007. 66(2): p. 456-466.

290. Grigorenko, B.L., A.V. Nemukhin, I.A. Topol, R.E. Cachau, and S.K. Burt, QM/MM Modeling the Ras-GAP Catalyzed Hydrolysis of Guanosine Triphosphate. Proteins-Structure Function and Bioinformatics, 2005. 60(3): p. 495-503.

291. Mayer, J.A. and K.J. Amann, Assembly Properties of the Bacillus Subtilis Actin, MreB. Cell Motility and the Cytoskeleton, 2009. 66(2): p. 109-118.

292. Dimasi, J.A., R.W. Hansen, H.G. Grabowski, and L. Lasagna, Cost of Innovation in the Pharmaceutical-Industry. Journal of Health Economics, 1991. 10(2): p. 107142.

293. Adams, C.P. and V.V. Brantner, Spending on New Drug Development. Health Economics, 2010. 19(2): p. 130-141.

294. Feixas, F., S. Lindert, W. Sinko, and J.A. McCammon, Exploring the Role of Receptor Flexibility in Structure-Based Drug Discovery. Biophysical Chemistry, 2014. 186: p. 31-45.

295. Sotriffer, C.A., Accounting for Induced-Fit Effects in Docking: What Is Possible and What Is Not? Current Topics in Medicinal Chemistry, 2011. 11(2): p. 179-191.

296. Jhoti, H., S. Rees, and R. Solari, High-Throughput Screening and Structure-Based Approaches to Hit Discovery: Is There a Clear Winner? Expert Opinion on Drug Discovery, 2013. 8(12): p. 1449-1453. 
297. Lavecchia, A. and C. Di Giovanni, Virtual Screening Strategies in Drug Discovery: A Critical Review. Current Medicinal Chemistry, 2013. 20(23): p. 2839-2860.

298. Blundell, T.L., Structure-Based Drug Design. Nature, 1996. 384(6604): p. 23-26.

299. Yang, Y., F.C. Lightstone, and S.E. Wong, Approaches to Efficiently Estimate Solvation and Explicit Water Energetics in Ligand Binding: The Use of Watermap. Expert Opinion on Drug Discovery, 2013. 8(3): p. 277-287.

300. Abel, R., T. Young, R. Farid, B.J. Berne, and R.A. Friesner, Role of the ActiveSite Solvent in the Thermodynamics of Factor Xa Ligand Binding. Journal of the American Chemical Society, 2008. 130(9): p. 2817-2831.

301. Rossato, G., B. Ernst, A. Vedani, and M. Smiesko, Acquaalta: A Directional Approach to the Solvation of Ligand-Protein Complexes. Journal of Chemical Information and Modeling, 2011. 51(8): p. 1867-1881.

302. Shan, Y., A. Arkhipov, E.T. Kim, A.C. Pan, and D.E. Shaw, Transitions to Catalytically Inactive Conformations in Egfr Kinase. Proceedings of the National Academy of Sciences, 2013. 110(18): p. 7270-7275. 